\title{
Experimental Model for Double Concave Sliding Bearings
}

\author{
A Project \\ Presented to the \\ Faculty of \\ California State Polytechnic University, Pomona
}

\author{
In Partial Fulfillment \\ Of the Requirements for the Degree \\ Master of Science \\ In \\ Civil Engineering
}

By

Arsen Adzhemyan

2017 


\section{SIGNATURE PAGE}

PROJECT: $\quad$ Experimental Model for Double Concave Sliding Bearings

AUTHOR: $\quad$ Arsen Adzhemyan

DATE SUBMITTED: $\quad$ Spring 2017

Civil Engineering Department

Giuseppe Lomiento

Project Committee Chair

Civil Engineering Department

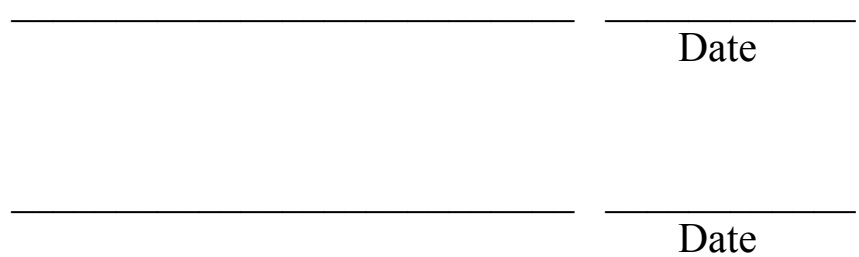

Mikhail Gershfeld

Civil Engineering Department 


\section{Declaration of Authorship}

I, Arsen ADZHEMYAN, declare that this project titled, "Experimental Model for Double Concave Sliding Bearings" and the work presented in it are my own. I confirm that:

- This work was done wholly or mainly while in candidature for a research degree at this University.

- Where any part of this project has previously been submitted for a degree or any other qualification at this University or any other institution, this has been clearly stated.

- Where I have consulted the published work of others, this is always clearly attributed.

- Where I have quoted from the work of others, the source is always given. With the exception of such quotations, this project is entirely my own work.

- I have acknowledged all main sources of help.

- Where the project is based on work done by myself jointly with others, I have made clear exactly what was done by others and what I have contributed myself.

Signed:

Date: 
California Polytechnic State University, Pomona

\author{
Abstract \\ Dr. Giuseppe Lomiento \\ Civil Engineering Department \\ Master's Degree in Structural Engineering \\ Experimental Model for Double Concave Sliding Bearings \\ by Arsen ADZHEMYAN
}

This study focuses on the performance of sliding friction seismic base isolation systems. Full-scale mono-directional tests are performed on double concave friction bearings in accordance with ASCE7-10 testing protocols. An existing experimental model for a single pendulum isolators is extended to double concave sliding bearings. The friction coefficient model is represented in terms of the applied vertical load, velocity, and cycling. Independent functions for each of these effects are calibrated and validated using test data to represent the observed variations in variations in friction coefficient. The applicability of the model to the double concave isolators is discussed, in comparison with earlier results on the single concave isolators. The feasibility of use of experimental data from current testing protocols for the calibration of the model is also addressed, and recommendations are provided. 


\section{Acknowledgements}

I would like to thank my colleagues, friends and family who supported me during my education. I am very grateful to all faculty of Civil Engineering Department of California Polytechnic State University, Pomona for the knowledge and inspiration they shared with me. Special thanks to Dr. Giuseppe Lomiento, who introduced me to the subject of seismic isolation among others, and always could help with advice in and outside of academia. Also, I would like to thank professor Mikhail Gershfeld, who shared his valuable knowledge and experience, and always was kind and patient enough to answer all of my questions. 


\section{Contents}

Declaration of Authorship $\quad$ i

$\begin{array}{ll}\text { Abstract } & \text { ii }\end{array}$

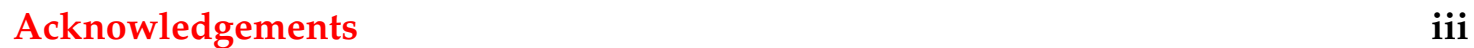

Contents $\quad$ iv

List of Figures $\quad$ vi

List of Tables $\quad$ viii

List of Abbreviations $\quad$ ix

Physical Constants $\quad$ x

List of Symbols $\quad$ xi

1 Introduction 1

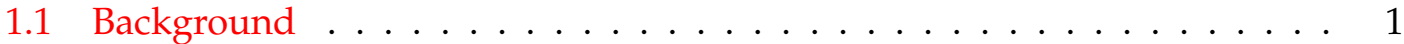

1.2 Problem Statement . . . . . . . . . . . . . . . . . . . 7

2 Testing Facilities 9

2.1 Shake Table Facilities . . . . . . . . . . . . . . . . . . . . . . . . 9

2.2 Testing Protocols . . . . . . . . . . . . . . . . . 10

3 Experimental Tests $\quad 18$

3.1 Experimental Data . . . . . . . . . . . . . . . . . . . . 19

3.2 Data Processing . . . . . . . . . . . . . . . . 20

4 Model Calibration $\quad 23$

4.1 Design Assumptions . . . . . . . . . . . . . . . . . . . 23

4.2 Vertical Load Effect . . . . . . . . . . . . . . . . . . . . 23

4.3 Velocity Effect . . . . . . . . . . . . . . . . . 27

4.4 Cycling Effect . . . . . . . . . . . . . . . . . . . . . 29

4.5 Combined Model . . . . . . . . . . . . . . . . . . . . . . 31

5 Model Validation $\quad 33$

5.1 Investigation Results . . . . . . . . . . . . . . . . . . . 33

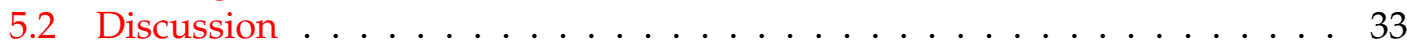

5.2.1 Adequacy of Testing Protocols . . . . . . . . . . . . . 37 
6 Conclusion 39

A Model Verification Plots $\quad 40$ 


\section{List of Figures}

1.1 Schematic and force-displacement plots for various isolator types (Matsagar and Jangid, 2008). . . . . . . . . . . . . . . . . . . 2

1.2 Schematic of single concave friction isolator. . . . . . . . . . . . 2

1.3 Effects of vertical load (A) and test velocity (B) on peak shear force(Benzoni et al., 2011). . . . . . . . . . . . . . . . . . . . 4

1.4 Experimental friction coefficient-displacement loops: (A) $P=15 \mathrm{MPa}, v=1.27$ $\mathrm{mm} / \mathrm{s}$, (B) $P=30 \mathrm{MPa}, v=1.27 \mathrm{~mm} / \mathrm{s}$, (C) $P=15 \mathrm{MPa}, v=100 \mathrm{~mm} / \mathrm{s}$, (D) $P=30 \mathrm{MPa}, v=100 \mathrm{~mm} / \mathrm{s}$ (Lomiento et al., 2013). . . . . . . . . . . . . 5

1.5 Schematic of double (A) and triple (B) pendulum sliding isolators. . . . . . 6

3.1 Theoretical behavior of concave sliding isolator: (A) force equilibrium and (B) force-displacement plot . . . . . . . . . . . . . . . . . . . . 18

3.2 Typical test data . . . . . . . . . . . . . . . . . . . . . 21

3.3 Typical plots obtained during the analysis . . . . . . . . . . . . . . 21

4.1 Hysteresis plots for bearing 16 isolator under different vertical loads . . . 24

4.2 Data selection regions . . . . . . . . . . . . . . . 25

4.3 Velocity effect function . . . . . . . . . . . . . . . . . . 26

4.4 Data outliers for bearing 18 isolator . . . . . . . . . . . . . 26

4.5 Experimental values of the friction coefficient $\mu$ vs. velocity presented by Lomiento et al. (2013). . . . . . . . . . . . . . . . . . . . . 27

4.6 Variation of the friction coefficient with sliding velocity, air temperature and bearing pressure, for non-lubricated interfaces. Comparison between analytical laws and experimental results. Air temperature equal to: (A) $-10^{\circ} \mathrm{C}$, (B) $20^{\circ} \mathrm{C}$, and (C) $50^{\circ} \mathrm{C}$ (Dolce et al., 2005) . . . . . . . . . . . 28

4.7 (A) Experimental variation of friction vs velocity, (B) Comparison of experimental data and proposed model during velocity effect calibration . . 29

4.8 Degradation of frictional properties with increase in sliding work (Chang et al., 1990). . . . . . . . . . . . . . . . . . 30

4.9 Correlation of function $z(c)$ with the experimental values of the cycling variable $c$ : (a) $p=15 \mathrm{MPa}$, (b) $p=30 \mathrm{MPa}$, (c) $p=60 \mathrm{MPa}$ presented by Lomiento et al. (2013). . . . . . . . . . . . . . . . . . . . . . . 30

4.10 Actual and equivalent uniformly distributed heat flux in the single concave sliding isolator in the time interval $d t$. (Lomiento et al., 2013). . . . . 30

4.11 Comparison of experimental data and proposed model . . . . . . . . 32

5.1 Comparison of experimental data and proposed model for bearing 16 . . 34

5.2 (A) A sudden drop in friction due to isolator geometry, and (B) zoomed in look for bearing 16 run $10 \ldots \ldots \ldots \ldots$ 
5.3 Diagram of the slider illustrating the mid-joint . . . . . . . . . . 36

5.4 (A) Areas affected by the velocity and cycling effects, and (B) zoomed in look for bearing 16 run $9 \ldots \ldots$. . . . . . . . . . . . . 37

5.5 (A) Areas of hysteresis loop affected by the velocity effect and underestimated by the proposed model, and (B) zoomed in look for bearing 16 run

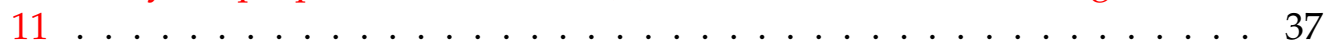

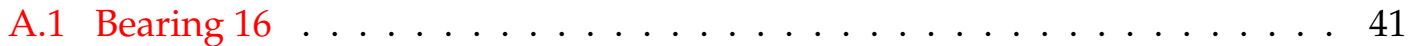

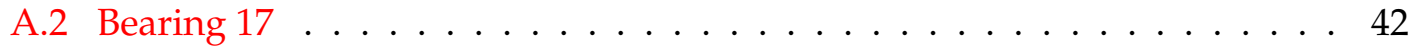

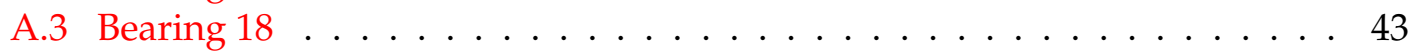

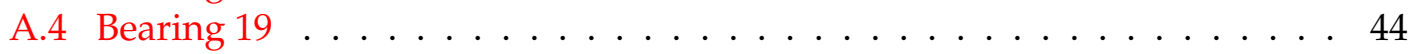

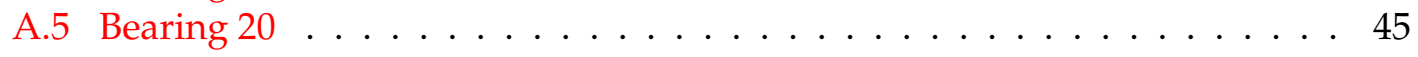

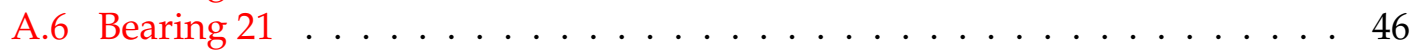

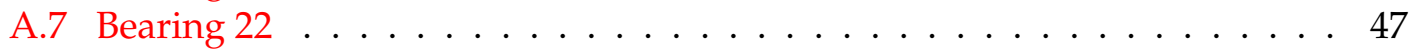


viii

\section{List of Tables}

2.1 SRMD Technical Specifications . . . . . . . . . . . . . . . . . . . 10

2.2 Sequence and cycles of prototype tests for AASHTO . . . . . . . . . . . . . 13

2.3 System adequacy requirements for AASHTO . . . . . . . . . . . . . . . . . 14

2.4 Sequence and cycles of prototype tests for ASCE 7-10 . . . . . . . 16

2.5 System adequacy requirements for ASCE7-10 . . . . . . . . . . . 17

3.1 Summary of Prototype Testing . . . . . . . . . . . . . . 22

5.1 Final values of constant coefficient and isolator parameters . . . . . . . . 33 


\title{
List of Abbreviations
}

\author{
DOF Degree Of Freedom \\ EDC Energy Dissipated per Cycle \\ FPS Friction Pendulum System \\ HDRB High Damping Rubber Bearing \\ LRB Lead Rubber Bearing \\ PTFE Polytetrafluoroethylene \\ SEAONC Structural Engineering Association of Northern California \\ SRMD Seismic Response Modification Device \\ UBC Uniform Building Code
}




\title{
Physical Constants
}

\author{
Gravitational acceleration $\quad g=9.81 \mathrm{~m} / \mathrm{s}^{2}$ \\ $\mathrm{Pi}$ \\ $\pi=3.1415$
}




\section{List of Symbols}

$\begin{array}{lll}A & \text { radius of the sliding surface of an isolator } & \mathrm{m} \\ a & \text { radius of a slider in an isolator } & \mathrm{m} \\ c(t) & \text { function describing heat distribution due to cycling } & \mathrm{N} / \mathrm{s} \\ c_{r e f} & \text { reference cycling coefficient } & \mathrm{N} / \mathrm{s} \\ d & \text { displacement } & \mathrm{N} \\ F & \text { force } & \\ f_{W}(W) & \text { vertical load effect function } & \\ f_{v}(v) & \text { velocity effect function } & \mathrm{Pa} \\ f_{c}(c) & \text { cycling effect function } & \mathrm{m} \\ p & \text { pressure } & \mathrm{S} \\ R & \text { radius } & \mathrm{m} / \mathrm{s} \\ T & \text { period of vibration } & \mathrm{m} / \mathrm{s} \\ v & \text { velocity } & \mathrm{N} \\ v_{r e f} & \text { reference velocity characterizing the variation rate of friction coefficient } \mu \\ W & \text { weight } & \mathrm{N} \\ W_{r e f} & \text { reference weight value used in the vertical load effect function } f_{W}(W) & \\ \beta & \text { exponent controlling the shape of the cycling effect function } f_{c}(c) & \\ \gamma & \text { ratio between the fast-motion and the slow-motion coefficient of friction } & \mathrm{rad} \mathrm{s}-1 \\ \omega & \text { angular frequency } & \\ \mu & \text { coefficient of friction } & \\ \mu_{s, 0} & \text { theoretical slow-motion coefficient of friction under no vertical load } & \end{array}$




\section{Chapter 1}

\section{Introduction}

\subsection{Background}

In recent decades, there has been an increase in the use of base isolators for seismic protection of critical structures throughout the world (Kelly, 1994; Warn and Ryan, 2012; Buckle, 2000). Applications include Stravos Niarchos Foundation Cultural Center in Athens, the Louvre Museum in Abu Dhabi, Los Angeles City Hall, and a number of bridges with Japan and China leading in the number of seismically isolated structures. Based on recorded earthquake data, structures equipped with base isolation technology proved to be more effective in dissipating seismic energy and resisting significant damages compared to traditionally-designed structures (Benzoni et al., 2011). Retrofit techniques involving base isolation systems have also been extensively investigated. Matsagar and Jangid (2008) examined the performance of elastometric bearings and concave sliding isolators for retrofit of three types of structures: historic buildings, bridges, and liquid storage tanks. The study determined both types of seismic isolation systems to be effective in reducing inertial forces on the previously mentioned types of structures from $20 \%$ to $70 \%$. Notable examples of structures retrofitted with friction pendulum system (FPS) include Benicia-Martinez Bridge retrofitted with 24 FPS isolators, the historic U.S. Court of appeals building in San Francisco, and the San Francisco Airport International Terminal that was damaged during the Loma Prieta earthquake in 1989 and retrofitted using 256 FPS isolators (Lomiento et al., 2013).

Overall, there have been proposed six main sliding base isolation systems with restoring force, two of which reached the stage of implementation (Dolce et al., 2005). Among several industry-accepted designs, concave sliding isolators (Figure 1.1c) offer larger displacement capacity with reduced thickness when compared to High Damping Rubber Bearing (HDRB) (Figure 1.1a) and Lead Rubber Bearing (LRB) (Figure 1.1b) isolators (Lomiento et al., 2013).

A single concave sliding isolator consist of a sliding concave spherical surface that supports a steel plate with housing and a slider with composite polymer lining (Figure 1.2). In structures protected with this technology, the seismic isolation is achieved by shifting the natural period of vibration of the superstructure while sliding. In this case, natural period of vibration depends only on the radius of curvature of the concave surface and not on the supported mass (Zayas et al., 1990). The relationship between the period of vibration of the structure during the sliding motion $(T)$ and the radius of curvature of the isolator surface $(R)$ is presented below:

$$
T=2 \pi \sqrt{R / g}
$$




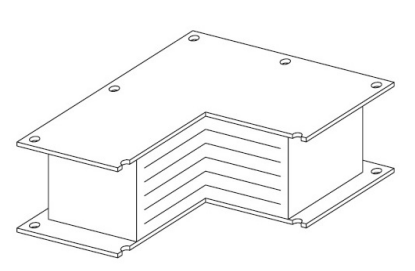

(A) HDRB

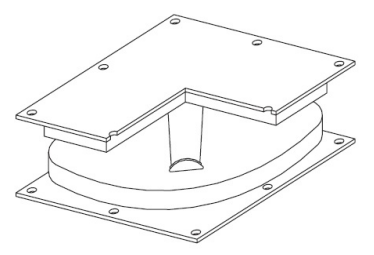

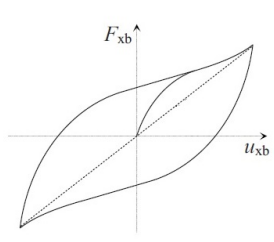

(C) FPS
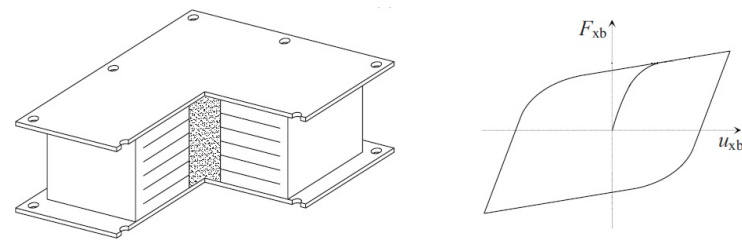

(B) LRB

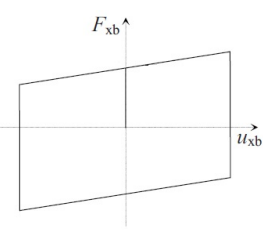

FIGURE 1.1: Schematic and force-displacement plots for various isolator types (Matsagar and Jangid, 2008).

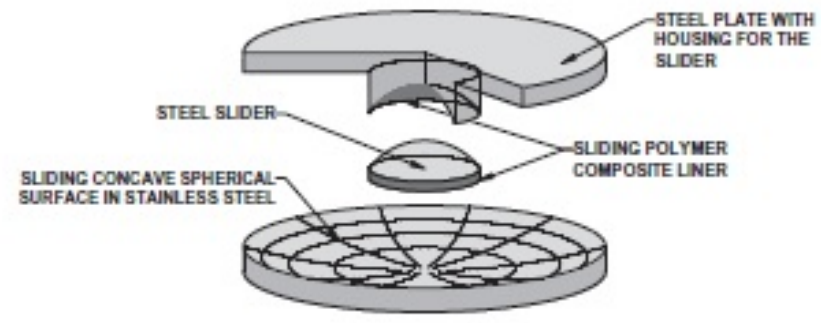

FIGURE 1.2: Schematic of single concave friction isolator.

where $g$ is the acceleration due to gravity.

Succeeding the original model of a two-story frame structure (Zayas, 1987), a variety of experimental and numerical studies have been conducted to evaluate the performance of sliding isolators. A shake-table study of the concave sliding isolation system installed in a six-story, quarter-scale model of a 52-kip structure discovered that the isolators were highly effective in protecting the frame-type structures from extreme seismic-loading conditions and high inner-story drifts (Mokha et al., 1991). The numerical model predicted forces and displacements with $20 \%$ accuracy. Tsopelas et al. (1996) conducted an experimental comparison of a seismically isolated bridge model and a comparable non-isolated bridge. The study found a significant reduction in inertia forces when isolators were used. These early studies assumed that the friction coefficient $\mu$ complies with the Coulomb friction law (i.e. friction remains constant throughout the motion). Actual experimental observations reported variations in friction. Chang et al. (1990) proposed an analytical model for Teflon stainless steel interfaces that included the effects of normal pressure, sliding distance, sliding velocity, normal pressure rate, sliding work, and histories of normal pressure and sliding velocity. The model was applicable to only mono-directional tests. Later studies primarily focused 
on analyzing and improving the originally proposed simplified models. Mosqueda et al. (2004) used a rigid-frame model to calibrate a constant coefficient of friction for rate-independent plasticity model of concave friction bearing isolators. A finite element model by Tsai (1997) assessed local bending moment effects and variation of vertical force for mono- and bi-directional motion. The model also presented some significant discoveries regarding Teflon metal interfaces:

- The reciprocal of quasi-static friction coefficient is linearly proportional to the applied normal pressure;

- The static friction force is higher than the dynamic friction force;

- The magnitude of the resultant of the dynamic friction force depends on both the magnitude of the instantaneous velocity and the applied normal pressure;

- At the same normal pressure and sliding velocity, the dynamic friction force decreases with the increase of the sliding cycles, and will approach to a stabilized hysteresis loop with a rectangular shape.

Studies that analyzed sliders lined with fluoropolymers such as the PTFE also reported dependency of friction on contact pressure and sliding velocity. Constantinou et al. (1990) proposed a mathematical model representing the uni- and multi- directional behavior of Teflon sliding bearing isolators. The study reported inconsistencies with Coulomb's constant friction model and a low sensitivity of Teflon bearings to the excitation frequency. Another study by Mokha et al. (1993) presented experimental results for Teflon bearings under simultaneous compression and high velocity multi-directional motion. The authors reported a bidirectional interaction between orthogonal components of the friction force. A series of experiments consisting of the application of nine pairs of horizontal earthquake motions to a structure isolated on forty-five sliding bearings were performed. Comparison of experimental results to a model which neglects the effects of bidirectional interaction highlight the underestimate of the slider displacement and the overestimate of the structure's base shear. Subsequent research further investigated the behavior of sliding bearings under dynamic loads. Three combinations of PTFE bearings were tested under excitation frequencies ranging from $0.02 \mathrm{~Hz}$ to 5 $\mathrm{Hz}$ (Bondonet and Filiatrault, 1997). The study found a high initial friction response at frequencies over $1 \mathrm{~Hz}$ before the system approached a steady state behavior after several cycles. A model implemented by Almazán et al. (1998) was capable of accounting for the uplift and vertical impact of the slider. Deb and Paul (2000) investigated the nonlinear dynamic response of buildings isolated with Teflon-stainless steel devices under bi-directional motion. It was observed that bi-axial interaction has a considerable effect on the performance of structures utilizing sliding elastometric bearings under bidirectional motion. An analytical formulation including $P-\Delta$ effects was described in Almazán and Llera (2002). The study concluded that models of structures under small-displacement impulsive loads may be up to $20 \%$ off in global response and up to $50 \%$ off in local response such as the normal force in the isolators or inter-story drifts. Later study by Chang and Spencer (2010) proposed the use of low-force hydraulic actuators in concave friction isolators to address excessive displacements of a superstructure caused by seismic isolation. The system incorporated electronic controllers, which 


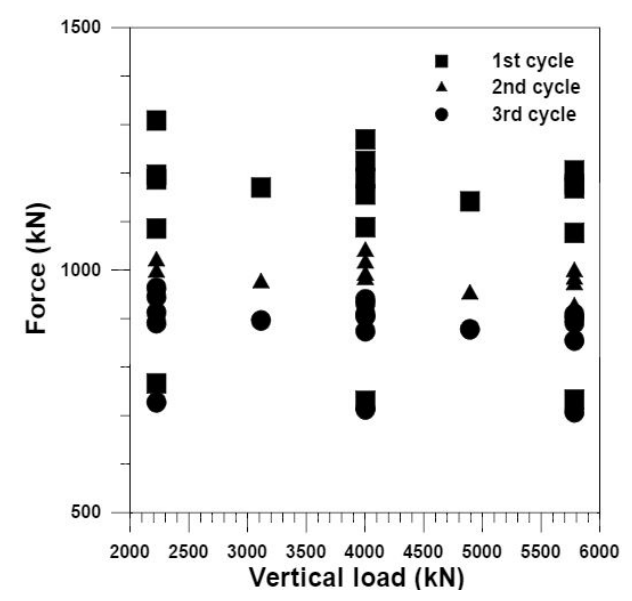

(A)

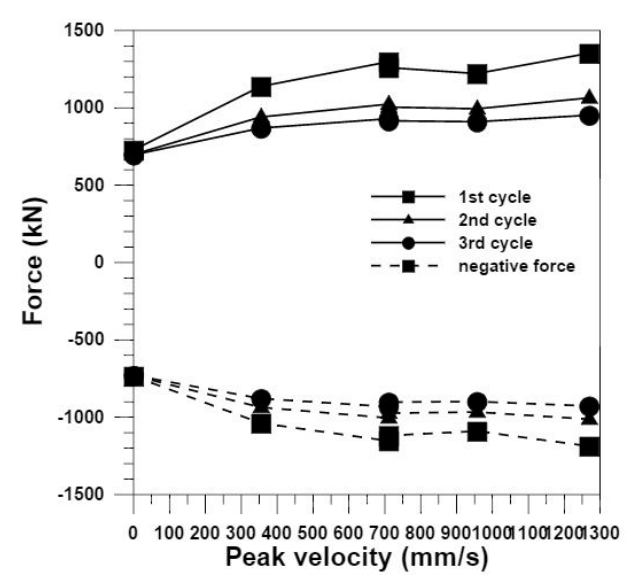

(B) Vertical load $=4004 \mathrm{kN}$

FIGURE 1.3: Effects of vertical load (A) and test velocity (B) on peak shear force(Benzoni et al., 2011).

can pose a possible issue if the electricity supply is interrupted during an earthquake. Retrofit techniques involving base isolation systems have also been extensively investigated. Matsagar and Jangid (2008) examined the performance of elastometric bearings and concave sliding isolators for retrofit of three types of structures: historic buildings, bridges, and liquid storage tanks. The study determined both types of seismic isolation systems to be effective in reducing inertial forces on the previously mentioned types of structures from $20 \%$ to $70 \%$.

Similarly to aforementioned studies, Benzoni et al. (2011) presented comparable dependency of friction coefficient on variations of the vertical loads and peak velocity when using single pendulum devices with hydrocarbon high-strength polymer sliding surfaces (Figure 1.3). The study also noted that a significant reduction in energy dissipation was observed with increased number of repetition cycles. Modern testing facilities allowed to conduct full-scale multi-directional tests on a variety of isolators attempting to simulate realistic seismic conditions and verify existing mathematical models. Comparison of experimental results with numerical simulations exposed the deficiencies of analytical solutions and simplified Coulomb theory for sliding bodies. A model described by Lomiento et al. (2013) based on a large set of experimental data was able to evaluate the influence of several main effects on the coefficient of friction $(\mu)$. The study identifies four main effects:

- "Breakaway effect", i.e. the sudden increase of $\mu$ at the beginning of each motion and at each direction reversal. The increased $\mu$ in the beginning of motion is know as a static $\mu$ and is often referred to as a breakaway coefficient of friction. The increase of $\mu$ at each change of direction of motion is typically referred to as stickslip. It is characterized by a short duration increase of $\mu$ followed by a rapid drop. Both static $\mu$ and stick-slip are caused by a momentary sticking of the interfaces and acceleration impulses.

- "Load effect", i.e. the reduction of $\mu$ for increasing contact pressure; 


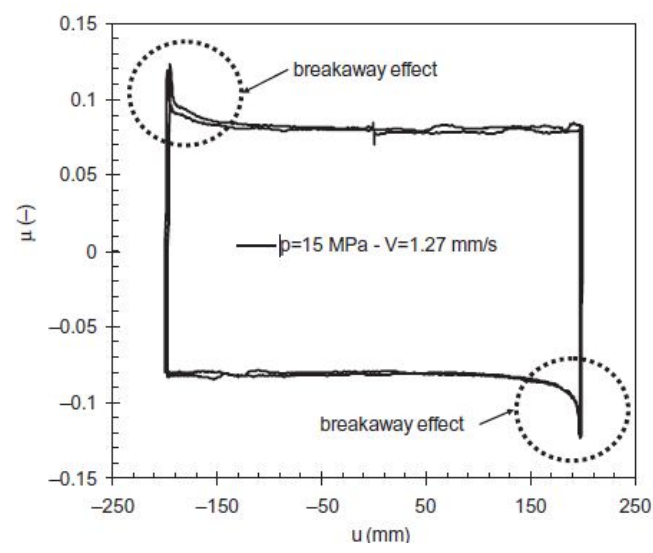

(A)

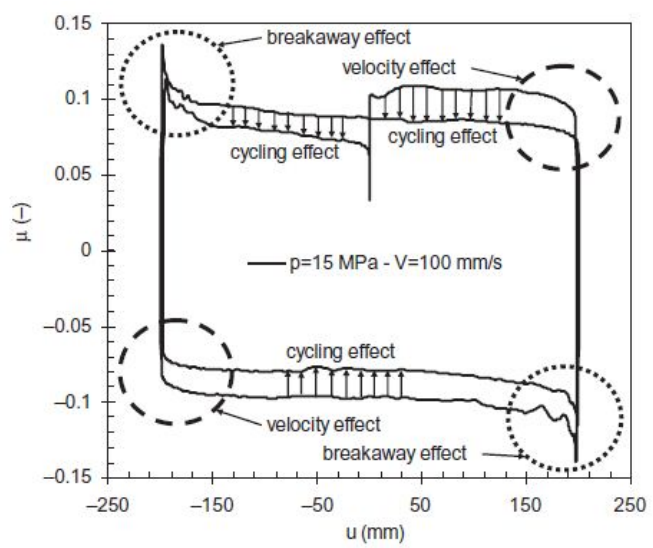

(C)

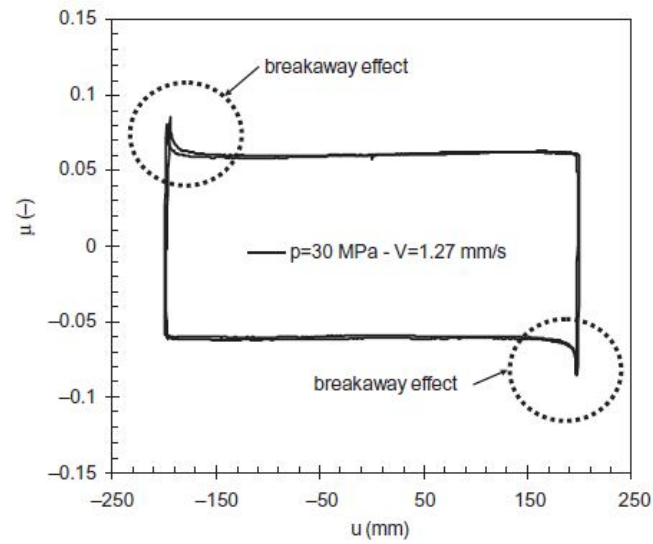

(B)

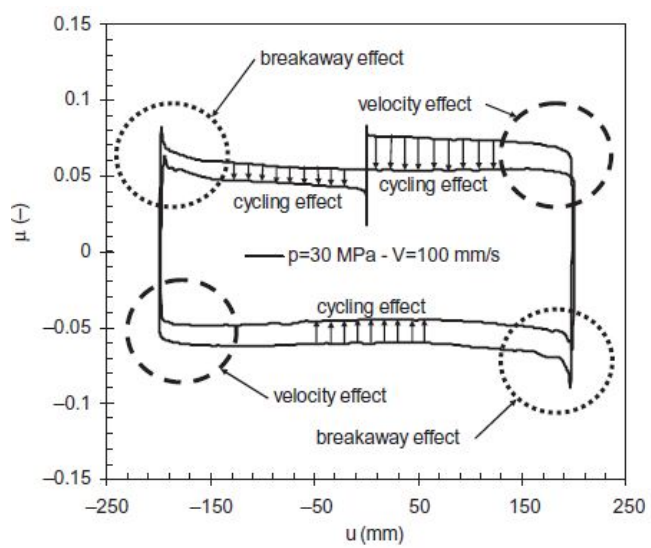

(D)

FIGURE 1.4: Experimental friction coefficient-displacement loops: (A) $P=15 \mathrm{MPa}, v=1.27 \mathrm{~mm} / \mathrm{s}$, (B) $P=30 \mathrm{MPa}, v=1.27 \mathrm{~mm} / \mathrm{s}$, (C) $P=15 \mathrm{MPa}$, $v=100 \mathrm{~mm} / \mathrm{s}$, (D) $P=30 \mathrm{MPa}, v=100 \mathrm{~mm} / \mathrm{s}$ (Lomiento et al., 2013).

- "Cycling effect", i.e. the continued reduction of $\mu$ with the fast repetition of cycles due to the increasing temperature of the sliding interface produced by frictional heating;

- "Velocity effect", i.e. the gradual increase of $\mu$ with the increasing sliding velocity.

These effects are best described by comparison of friction coefficient-displacement loops (Figure 1.4). The reduction in friction coefficient $\mu$ with increased vertical pressure $p$ (load effect) is present for both low- and high- velocity plots (Figure 1.4a vs Figure 1.4b and Figure 1.4c vs Figure 1.4d, respectively). Velocity effect is evident when examining high velocity plots and focusing on regions of maximum displacement where the slider is coming to a stop. The decrease in velocity is accompanied by a reduction in friction coefficient $\mu$ (Figure 1.4c and Figure 1.4d). Breakaway effect is represented by a spike in friction at each reversal of motion of the slider. Similarly to the velocity effect, cycling effect is the most evident during high velocity tests. It is characterized by a reduction of the area inside the friction coefficient-displacement loop with each cycle (Figure 1.4c and Figure 1.4d). 


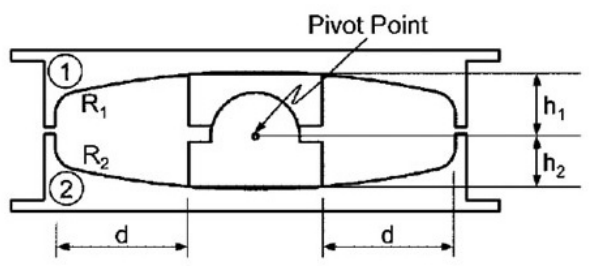

(A)

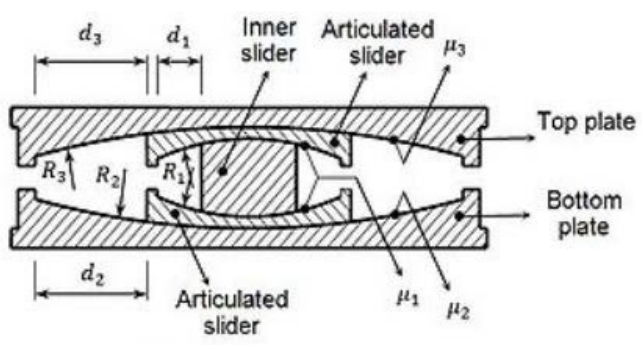

(B)

FIGURE 1.5: Schematic of double (A) and triple (B) pendulum sliding isolators.

The authors expressed the coefficient of friction $\mu$ as a product of three independent functions each associated with one of the described effects:

$$
\mu(W, c, v)=f_{W}(W) \cdot f_{c}(c) \cdot f_{v}(v)
$$

where $f_{W}$ is a function representing load effect, $f_{c}$ - cycling effect, and $f_{v}$ - velocity effect, respectively. The model was calibrated and verified against experimental data.

Single pendulum isolators evolved into double- and triple-pendulum devices due to significantly increased displacement capacity compared to single pendulum isolators of the same bearing dimensions. Double pendulum friction isolators are composed of two concave sliding surfaces and a slider in between and are capable of double the relative displacement range (Figure 1.5a). Triple pendulum friction isolators consist of a double pendulum isolator where the inner slider is replaced with another double pendulum device of different frictional characteristics (Figure 1.5b). The internal slider is designed to be activated when the external components reach the maximum displacement capacity. This mechanism can provide hardening behavior.

Fenz and Constantinou (2006) stated that the double concave friction bearing exhibits a behavior similar to a single pendulum isolator with effective radius of curvature equal to the sum of the radii of curvature of two concave surfaces and modified for the height of the slider. Authors also discovered that friction is equal to the average value of the coefficients of friction of each concave interfaces $\left(\mu_{1}\right.$ and $\left.\mu_{2}\right)$. Fenz and Constantinou (2006) also proposed using different radii of curvature for the two concave surfaces of the double concave friction sliding isolator. The study revealed that

- double sliding pendulum exhibits rigid bilinear hysteretic behavior when $\mu_{1} \neq \mu_{2}$ which is reduced to a rigid linear behavior for when $\mu_{1}=\mu_{2}$;

- the effective radius of the double isolator is reduced by slider height;

- the slider experiences rotation only when $\mu_{1} \neq \mu_{2}$. The relationship between radii of curvature has no effect on slider rotation;

- the behavior of double sliding pendulum system can be represented by two single pendulum systems connected in series with a mass in between to simulate the slider. 
A similar approach was taken by Lu et al. (2006), who proposed using a varying radius of curvature of the spherical surface rather than a traditional constant radius. The study was conducted on a single concave sliding bearing and used a polynomial function to plot the radius. As a result of varying radius, the frequency of the isolator was not constant, and the low-frequency resonance due to long-period components of a near-fault earthquake can be prevented. Numerical study has demonstrated that, when subjected to a long-period pulse-like earthquake, the proposed isolator was able to simultaneously reduce the isolator drift and structural acceleration, as compared with those of a conventional concave friction sliding system.

Performance of structures equipped with double sliding pendulum technology has been further investigated. Tsai et al. (2008) proposed using a direction-optimized concave sliding bearings. The proposed device was a combination of a trench friction sliding system and a concave sliding isolator. The authors stated that the isolator can continually change the natural period of the structure during sliding and adjust the capacity of the bearing displacement and damping effect by changing the angle between the articulated slider and trench concave surface during earthquakes.

Morgan and Mahin (2010) proposed using a multi-stage pendulum system to capture a wider range of seismic activities. The system utilized three independent friction pendulum mechanisms whose stiffness and damping characteristics could be selected based on multiple levels of seismic hazard. The study demonstrated the benefits of such systems and trade-offs between isolator displacement, peak inter-story drifts, and peak floor accelerations.

Triple friction pendulum isolator have not been extensively studied. Becker and Mahin (2012) implemented a general model for triple concave sliding pendulum system that was capable of capturing bi-directional motion and explicitly track the movement of internal components. The model was insensitive to variations in bearing properties due to its general nature.

Most of the aforementioned studies rely on simplified models of the coefficient of friction, including at most the velocity and the vertical load effects, but always neglecting the degradation of frictional properties due to the increase in heat. Similarly, only the variations in friction coefficient due to the velocity and the vertical load effects are implemented into the commercial software such as SAP2000(C)and OpenSees(C). This is currently accepted by Codes as a state-of-practice, but does not reflect the most recent experimental evidence.

\subsection{Problem Statement}

The performance of devices (at least for single and double pendulum configuration) has been extensively investigated with large experimental campaigns, greatly contributed by the Caltrans SRMD Testing Facility at the University of California San Diego. The unique experimental database containing results from tests of full-scale isolators across a range of realistic vertical loads and sliding velocities for multi- directional excitations (Benzoni et al., 2011; Lomiento et al., 2012), indicated that the response prediction based on commonly accepted models and code requirements for concave sliding isolators can be improved by addressing previously described major phenomena observed during laboratory tests (Section 1.1). Early protocols addressing base isolation techniques were introduced in 1980 by Structural Engineering Association of Northern 
California (SEAONC), and after several modifications were finally adopted by Uniform Building Code (UBC) in 1991 (Kelly, 1994). The concave sliding system by Zayas (1987) was introduced after the initial development of the code requirements governing seismic base isolation systems. It must be noted that even recent code requirements for device performance characterization are still based on the early knowledge of this technology (i.e. rubber isolation devices) (CEN/TC 340, 2006). A contributing factor could be the extreme simplification of early scientific work in the description of the devices' performance, namely, the assumption of constant coefficient of friction $\mu$. This attempt to simplify the performance of friction-based isolators by neglecting the degradation of frictional properties due to vertical load, velocity, and cycling effects is still detectable in literature even for isolators significantly more complex than the single pendulum bearing (e.g. devices based on multiple friction surfaces).

The current testing protocols and acceptance criteria for friction-based devices are not optimized to obtain and validate the main performance characteristics of single and double concave friction bearings such as the reduction of EDC with the increase in the number cycles. The number of requested tests could be significantly reduced and/or reorganized in order to better qualify the devices in terms of performance variation due to varying axial load, velocity effects, cycling effects (thermal), asynchronous sliding and moment-shear interaction. Each of the aforementioned effects can be addressed by a single test (or a combination of tests for complex cases) and then combined into a single model capable of predicting system's behavior. Such a model would eliminate the need of conducting a series of tests at varying load and displacement increments.

The purpose of this paper is to determine whether current isolator testing protocols of ASCE 7 (2010) are correctly designed to provide the structural engineer with a comprehensive picture of the device performance that does not require an exercise of extrapolation and/or assessment for practical implementation. Another focus of this study is to determine the validity of the proposed numerical model for single concave sliding pendulum isolator by Lomiento et al. (2013) and to extend the model to a double pendulum system. The availability of the aforementioned numerical model that appears capable of matching the degradation of friction phenomena observed in single pendulum devices, is a solid starting point for the proposed research activity. The existing model needs of course to be validated and tuned with experimental data of double pendulum devices. The lack of an interpretative scheme (or a specific performance model) makes the interpretation of the results from very extensive test programs, quite inefficient because the single tests are not designed to isolate one (and possible only one) specific behavior. The design of testing procedures that minimize the interaction between performance characteristics will allow calibrating each single component of the phenomenological model. That is, each component included in the frictional model in Equation 1.2 can be calibrated separately and then combined into one model. This will guarantee an efficient transition from the acquisition of experimental data to the implementation of the critical results into numerical analyses of the structure under consideration. 


\section{Chapter 2}

\section{Testing Facilities}

\subsection{Shake Table Facilities}

There has been an increase in the number of experimental facilities specifically designed for testing of seismic isolation devices. The spread and availability of this technology, limited before to manufacturing plants, allowed to conduct research in universities and independent facilities. For the purposes of this project, full-scale isolator testing was conducted at Caltrans Seismic Response Modification Device (SRMD) Laboratory at the University of California, San Diego. The facility is equipped with a 6 DOFs shake table specifically designed for full-scale testing of seismic isolators, dampers and other energy dissipation devices (Benzoni and Seible, 1998). The laboratory occupies 3,200 $\mathrm{ft}^{2}$ (300 $\mathrm{m}^{2}$ ) of space and is serviced by a 20-ton (40-kip) overhead crane. The colossal forces required for testing are possible by the use of hydraulic actuators that use approximately 5,000 gallons (19,000 liters) of oil that is pressurized by nitrogen gas up to 5,000 psi (34 $\mathrm{MPa})$.

This state-of-the-art technology allows longitudinal displacement range of $+/-48$ inches $(1.22 \mathrm{~m})$, lateral displacement of $+/-24$ inches $(0.61 \mathrm{~m})$, and vertical displacement of $+/-5$ inches $(0.127 \mathrm{~m})$, all three parameters with an accuracy of $+/-2 \%$. The setup is able to produce a longitudinal force of 2,000 kips $(8,900 \mathrm{kN})$, lateral force of 1,000 kips $(4,450 \mathrm{kN})$, and a vertical force of $12,000 \mathrm{kips}(53,400 \mathrm{kN})$ to simulate the weight of a full-scale building. Maximum achievable velocity values are $+/-70 \mathrm{in} / \mathrm{s}(1,778$ $\mathrm{mm} / \mathrm{s}),+/-30 \mathrm{in} / \mathrm{s}(762 \mathrm{~mm} / \mathrm{s})$, and $+/-10 \mathrm{in} / \mathrm{s}(254 \mathrm{~mm} / \mathrm{s})$ for longitudinal, lateral and vertical directions, respectively. The accuracy of application for velocity parameters is $+/-10 \%$ (Benzoni and Seible 1998). Table 2.1 summarizes the technical parameters of the Caltrans SRMD system.

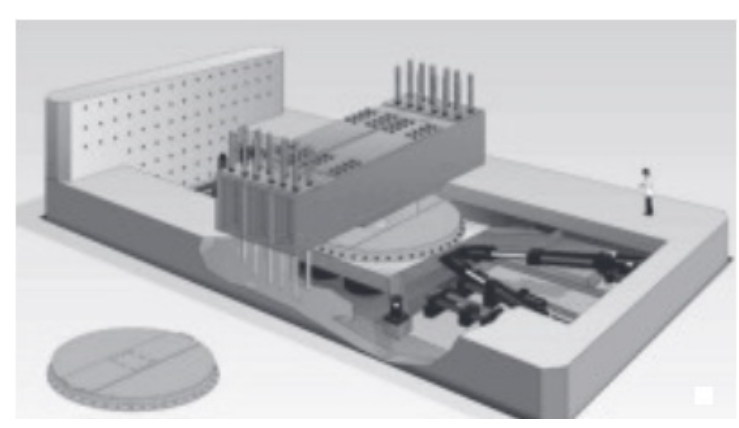

(A) SRMD test setup

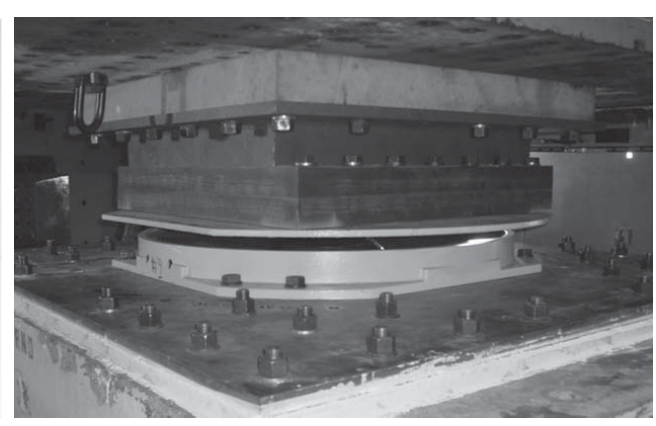

(B) Isolator installed on a shake table 


\begin{tabular}{|c||c|c|c|}
\hline Component & Capacity & $\begin{array}{c}\text { Accuracy of } \\
\text { Application }\end{array}$ & Accuracy of Readout \\
\hline \hline Vertical Force & $53,400 \mathrm{kN}(12,000 \mathrm{kips})$ & $\pm 5 \%$ & $0.5 \%$ full range \\
\hline Longitudinal Force & $8,900 \mathrm{kN}(2,000 \mathrm{kips})$ & & $1.0 \%$ full range \\
\hline Lateral Force & $4,450 \mathrm{kN}(1,000 \mathrm{kips})$ & & $1.0 \%$ full range \\
\hline Vertical Displacement & $\pm 0.127 \mathrm{~m}(5 \mathrm{in})$. & $\pm 2 \%$ & $1.0 \%$ full range \\
\hline Longitudinal Displacement & $\pm 1.22 \mathrm{~m}(48 \mathrm{in})$. & $\pm 2 \%$ & $1.0 \%$ full range \\
\hline Lateral Displacement & $\pm 0.61 \mathrm{~m}(24 \mathrm{in})$. & $\pm 2 \%$ & $1.0 \%$ full range \\
\hline Vertical Velocity & $\pm 254 \mathrm{~mm} / \mathrm{s}(10 \mathrm{in} / \mathrm{s})$ & $\pm 10 \%$ & \\
\hline Long. Velocity & $\pm 1,778 \mathrm{~mm} / \mathrm{s}(70 \mathrm{in} / \mathrm{sec})$ & $\pm 10 \%$ & \\
\hline Lateral Velocity & $\pm 762 \mathrm{~mm} / \mathrm{s}(30 \mathrm{in} / \mathrm{sec})$ & $\pm 10 \%$ & \\
\hline Height of Specimen & Up to $1.52 \mathrm{~m}(5 \mathrm{ft})$ & & \\
\hline Relative Platen Rotation & $\pm 2^{\circ}$ & & \\
\hline \hline
\end{tabular}

TABLE 2.1: SRMD Technical Specifications

The isolator devices are installed on the shake table testing machine in accordance with standard installation procedures for isolation devices. The isolator's bottom plate is connected to the shake table, and the top portion of the isolator above the slider is bolted to a reaction beam to simulate a fixed support condition. During each test run, the shake table is raised vertically to compress the isolator against the reaction beam to represent a vertical load imposed by a structure.

\subsection{Testing Protocols}

Current design standards such as ASCE 7 (2010), AASHTO (2012), and CEN/TC 340 (2006) are applicable to only two types of tests: Prototype (Type) and Production (Proof) tests. Prototype tests are typically completed during the transition between the Research and Development $(R \& D)$ phase and the Production and Commercialization $(P \& C)$ phase and are designed to confirm the device performance under a great variety of loads and displacements. Specific design parameters need to be established in order to qualify the device as either project-specific or assigned to a desired level of pre-qualification requested by the manufacturer or the end user. The Production Tests, on the other hand, are designed and used to validate and ensure the average performance of a batch of produced units, with reference to the prototype response. The level of applied actions is quite limited for these tests in order to maintain the device in working conditions and ready for installation (Benzoni et al., 2011). Many current codes do not present a complete set of requirements for a proper test execution. Benzoni et al. (2011) mentions that the request of a fixed number of fully reversed cycles at specified maximum displacement and peak velocity without indicating the shape of the displacement time history to be applied (sinusoidal, constant velocity etc.), is quite common. Response of devices that are sensitive to acceleration peaks can vary greatly for various shapes of the displacement curve; thus, this specification is necessary, especially for devices with many mechanical joints involved in the overall motion. The typical request of the repetition of a number of cycles at design displacement is also broadly applied to devices of the same 
family without consideration for the supplemental phenomena involved in a device's performance for large ranges of displacements. In this respect, a positive example is offered by the European Code EN15129 (CEN/TC 340, 2006) in which, for instance, it is stated that for viscous dampers " The Structural Engineer shall prescribe the acceptable variation of the output force due to changes in ambient or internal temperature, or due to causes as aging, wearing, etc". Modifications of the device performance due to thermal effects are, in this case, taken into account. Use of new technologies within a project requires an active participation of the designer in the phase of collection and interpretation of the documentation of the performance of a mechanical device to be included in the design. This phase should ideally be initiated during the selection process of the most appropriate technologies to be used, in close collaboration with manufacturers. Too often, unfortunately, this role is devolved to the manufacturer, based on the reason of lack of expertise on the part of the designer. Introducing a separation between the detailed knowledge of the overall structural concept and the specific device's performance is, however, extremely dangerous in the design of seismically isolated structures (Benzoni et al., 2011). In this respect, the codes tend to be used as missing link between the manufacturer's and the designer's specific expertise, with the assumption that the satisfaction of code requirements guarantees a minimum safety level. It is desired that professionals use codes as guidelines and develop a deeper understanding of the isolation technique's performance and limitations associated with it. Developing expertise beyond the basic code-mandated design requirements is necessary.

The extrapolation of performance results, obtained during laboratory tests on certain devices, to units of quite different dimensions and performance characteristics, is a highly debated subject. The European Code EN 15129 (CEN/TC 340, 2006) prescribes some limitation to this process, indicating, for instance, the modifications to an elastomeric isolator requiring a new set of prototype tests. It is, however, unquestionable the tendency, in practice, to propose this extrapolation process, disguised under the name of "pre-qualification". Reasons of concern for this approach are justified when, for instance, dealing with friction-based devices. It is typically believed that a friction coefficient $\mu$ between two materials is a material property and is independent of application. This misconception takes its roots from the times when Leonardo da Vinci first experimented with frictional properties of materials. Modern scientists agree that the friction coefficient is just a simplification used for convenience, describing a friction system, and not a material property. Hence, the assessment of a given performance based on the similitude of employed material, cannot be proposed when devices represent different systems. Several tests performed at the SRMD laboratory indicated, for instance, that friction coefficients obtained by tests on small sample of materials were not reproduced by tests on full-scale devices using identical materials. Despite that, current regulations allow reduced-scale specimen testing (ASCE 7, 2010).

Testing protocols only rarely contains specifications about the testing equipment. The existence of a current calibration of displacement transducers, load cells, pressure transducers etc., is generally implied, but neither procedures nor ranges of accuracy versus capacity, are provided for guidance. It is however desirable the initiation of a process of accreditation of dedicated facilities that could include, moreover, conditions of repeatability and traceability, procedures for data acquisition and data reduction etc. The development of specific regulations on this matter is considered a crucial step in 
view of an improved service toward the seismic isolation technology. The capability, robustness and reliability of testing equipment are directly linked with the requirements of specific testing protocols, such as the completion of tests with simultaneous application of components of motion in multiple directions (2D or 3D tests) which has recently generated an interesting debate, in Italy. It must be noted that the European code suggests multi -directional tests, but considers the limited availability of facilities with this performance. For this reason it allows substituting the multi -directional tests by $1 \mathrm{D}$ tests repeated in orthogonal directions of motion (CEN/TC 340, 2006). However, nowhere in the European code, and in any other existing code, is this simplification intended to question the significance of tests using multi-directional motions. Unfortunately, the importance of these tests was recently doubted by experts. On the contrary, the activity at the San Diego Laboratory can document not only the increasing interest of the community in the use of realistic type of motions, but also an extensive history of criticalities highlighted by multi-directional tests on every family of devices. As mentioned previously, in order to ensure desired performance, selected isolation products should be extensively tested under real conditions independently of what the code prescribes as minimum requirements.

Current AASHTO code requires 7 Prototype test in order to determine the system's behavior characteristics:

1. Thermal - Three fully reversed cycles of loads at a lateral displacement corresponding to the maximum thermal displacement. The test velocity shall not be less than 0.003 inches per minute

2. Wind and Braking - Twenty fully reversed cycles between limits of plus and minus the maximum load for a total duration not less than 40 seconds. After the cyclic testing, the maximum load shall be held for 1 minute

3. Seismic - Three fully reversed cycles of loading at each of the following multiples of the total design displacement: $1.0,0.25,0.50,0.75,1.0$, and 1.25 , in the sequence shown

4. Seismic - 20 cycles of loading at 1.0 times the design displacement. The test shall be started from a displacement equal to the offset displacement

5. Wind and Braking - Three fully reversed cycles between limits of plus and minus the maximum load for a total duration not less than 40 seconds. After the cyclic testing, the maximum load shall be held for 1 minute

6. Seismic Performance Verification - Three fully reversed cycles of loading at the total design displacement

7. Stability Verification - The vertical load-carrying elements of the isolation system shall be demonstrated to be stable under one fully reversed cycle. 


\begin{tabular}{|c|c|c|c|c|c|c|c|}
\hline Test & Type & Vertical Load & \# of cycles & Peak displacement & Peak Force & Velocity & Duration \\
\hline 1 & Thermal & $\mathrm{DL}$ & 3 & $\begin{array}{c}\text { Max thermal } \\
\text { displacement }\end{array}$ & - & $>0.003 \mathrm{in} / \mathrm{min}$ & - \\
\hline 2 & $\begin{array}{c}\text { Wind and } \\
\text { Braking }\end{array}$ & $\mathrm{DL}$ & 20 & - & $+/-\mathrm{WL}$ & - & $>40 \mathrm{sec}^{[1]}$ \\
\hline 3 & Seismic & $\mathrm{DL}$ & 3 & $\begin{array}{c}1.0 \mathrm{i}, 0.25 \mathrm{i}, 0.5 \mathrm{i}, \\
0.75 \mathrm{i}, 1.0 \mathrm{i}, 1.25 \mathrm{i}\end{array}$ & - & - & - \\
\hline 4 & Seismic & $\mathrm{DL}$ & 20 & $1.0 \mathrm{i}(2)$ & - & - & - \\
\hline 5 & $\begin{array}{c}\text { Wind and } \\
\text { Braking }\end{array}$ & $\mathrm{DL}$ & 3 & - & $+/-\mathrm{WL}$ & - & $>40 \mathrm{sec}^{[1]}$ \\
\hline 6 & $\begin{array}{c}\text { Seismic } \\
\text { Performance } \\
\text { Verification }\end{array}$ & $\mathrm{DL}$ & 3 & $1.0 \mathrm{i}$ & - & - & - \\
\hline 7 & $\begin{array}{c}\text { Stability } \\
\text { Verification }\end{array}$ & $\begin{array}{c}1.2 \mathrm{DL}+\mathrm{LL}+\mathrm{OT}, \\
0.8 \mathrm{DL}-\mathrm{OT}\end{array}$ & 1 & - & - & - & - \\
\hline
\end{tabular}

Notes:

(1) After the cyclic testing, the maximum load shall be held for $1 \mathrm{~min}$.

(2) The test shall be started from a displacement equal to the offset displacement.

TABLE 2.2: Sequence and cycles of prototype tests for AASHTO

Table 2.2 summarizes main parameters and sequence of the tests. Three levels of vertical load are considered:

1. $D L$

2. $1.2 D L+L L_{S}+O T$

3. $0.8 D L-O T$,

where $D L$ - Dead Load, $L L_{S}$ - Seismic Live Load, $O T$ - additional vertical load on bearing resulting from overturning moment effect of horizontal loads.

AASHTO also considers 6 levels of displacement:

1. $1.0 \mathrm{i}$

2. $0.25 \mathrm{i}$

3. $0.5 \mathrm{i}$

4. $0.75 \mathrm{i}$

5. $1.0 \mathrm{i}$

6. $1.25 \mathrm{i}$,

where i - design displacement.

Systems Adequacy tests designed to confirm isolators' behavior to the Prototype tests are summarized in Table 2.3. Requirements for reference tests are established: 


\begin{tabular}{|c|c|c|}
\hline Req.\# & Reference Tests & System adequacy requirement \\
\hline 1 & $1,2,3,4,5,6,7$ & $\mathrm{~T}<6 \mathrm{sec}, \mathrm{F}_{\mathrm{i}}=\mathrm{W} / 80 \mathrm{~F}_{0.5 \mathrm{i}}$ \\
\hline 2 & 1 & $\mathrm{~F}_{\mathrm{p}, \max }<\mathrm{F}_{\text {design }}$ \\
\hline 3 & 2,5 & $\mathrm{i}_{\max }<\mathrm{i}$ \\
\hline 4 & 3 & $0.9 \mathrm{k}_{\text {design }}<\mathrm{k}_{\text {eff, ave }}^{[1]}<1.1 \mathrm{k}_{\text {design }}$ \\
\hline 5 & 3 & $\mathrm{k}_{\text {eff, min }} \geq 0.8 \mathrm{k}_{\text {eff, max }}[1]$ \\
\hline 6 & 4 & $\mathrm{k}_{\text {eff, } \min } \geq 0.8 \mathrm{k}_{\text {eff, max }}(2)$ \\
\hline 7 & 4 & $E D C_{\min } \geq \mathrm{EDC}_{\max }^{(2)}$ \\
\hline 8 & 7 & $\begin{array}{c}\text { All vertical load-carrying } \\
\text { elements of the isolation } \\
\text { system shall remain stable } \\
\text { (positive incremental stiffness) }\end{array}$ \\
\hline
\end{tabular}

Notes:

(1) The average effective stiffness shall be measured in the last three cycles of motion.

(2) At the discretion of the engineer, a larger variation may be accepted, provided that both the minimum and maximum values of effective stiffness are used in the design.

(3) Test specimens shall be visually inspected for evidence of significant deterioration. If any deterioration exists, then the adequacy of the test specimen shall be determined by the engineer.

TABLE 2.3: System adequacy requirements for AASHTO

1. $T<6 s e c, F_{i}=W / 80 F_{0.5 i}$

2. $F_{p, \max }<F_{\text {design }}$

3. $i_{\max }<i$

4. $0.9 k_{\text {design }}<k_{\text {eff,ave }}<1.1 k_{\text {design }}$

5. $k_{\text {eff,min }} \geq 0.8 k_{e f f, \max }$

6. $E D C_{\min } \geq E D C_{\max }$

7. All vertical load-carrying elements of the isolation system shall remain stable,

where $T$ - period corresponding to its tangent stiffness based on the restoring force alone at any displacement, $W$ - total vertical load for design of the isolation system including Deal and Seismic Live Loads, $F_{i}$ - force in the isolator unit at displacement $\Delta_{i}, i$ - design displacement, $F_{p, \max }$ - maximum positive force in an isolator unit for all cycles of prototype testing at a common displacement amplitude, $F_{\text {design }}$ - design force in an isolator unit, $i_{\max }$ - maximum measured displacement, $k_{\text {design }}$ - value of stiffness used in the design, and $k_{e f f, a v e}$ - average effective stiffness value. If compliance with all requirements is confirmed, the isolator system can be used further in the design.

ASCE7-10 proposes similar testing procedures. Three different levels of vertical loads are considered:

1. Vertical load level 1 (average): $1.0 D+0.5 L$ 
2. Vertical load level 2 (maximum): $1.2 D+1.0 E+1.0 L+0.2 S$

3. Vertical load level 3 (minimum): $0.9 D+1.0 E$,

where $D$ - Dead Load, $L$ - Live Load, $S$ - Snow Load, $E$ - Earthquake Load (Maximum Considered Earthquake (MCE)). A total of 8 tests need to be performed including repetitions of test N2 for 3 vertical loads, and of test N5 for 2 vertical loads.

Several displacement levels are required at each test cycle:

1. $0.25 D_{D}$

2. $0.5 D_{D}$

3. $1.0 D_{D}$

4. $1.0 D_{M}$

5. $1.0 D_{T M}$

6. $1.0 D_{T D}$,

where

$$
\begin{gathered}
D_{D}=\frac{g S_{D 1} T_{D}}{4 \pi^{2} B_{D}}-\text { design displacement, } \\
D_{M}=\frac{g S_{M 1} T_{M}}{4 \pi^{2} B_{M}}-\text { maximum displacement, } \\
D_{T D}=D_{D}\left[1+y \frac{12 e}{b^{2}+d^{2}}\right]-\text { total design displacement, } \\
D_{T M}=D_{M}\left[1+y \frac{12 e}{b^{2}+d^{2}}\right]-\text { total design displacement, }
\end{gathered}
$$

and

$$
T_{D}=2 \pi \sqrt{\frac{W}{k_{D \min } g}}
$$

- the effective period of the isolated structure at design displacement,

$$
T_{M}=2 \pi \sqrt{\frac{W}{k_{M \min } g}}
$$

- the effective period of the isolated structure at maximum displacement, $g$ - the acceleration due to gravity, $S_{D 1}$ - design 5\% damped spectral acceleration value at $1 \mathrm{sec}$ period, $B_{D}, B_{M}$ - numerical coefficients related to the effective damping of the isolation system at the design displacement $\beta_{D}$ and maximum displacement $\beta_{M}$,respectively, as specified in Table 17.5-1 of ASCE 7 (2010), where

$$
\beta_{D}=\frac{\Sigma E_{D}}{2 \pi k_{D \max } D_{D}^{2}},
$$




\begin{tabular}{|c|c|c|c|c|c|}
\hline Test & Vertical Load & \# of Cycles & Peak Displacement & Peak Force & Period \\
\hline 1 & $1.0 \mathrm{D}+0.5 \mathrm{~L}$ & 20 & - & $\mathrm{F}_{\mathrm{W}}$ & $\mathrm{T}_{\mathrm{D}}{ }^{[1]}$ \\
\hline 2 & \begin{tabular}{|c|}
$1.0 \mathrm{D}+0.5 \mathrm{~L}$ \\
$1.2 \mathrm{D}+1.0 \mathrm{E}+1.0 \mathrm{~L}+0.2 \mathrm{~S}$, \\
$0.9 \mathrm{D}+1.0 \mathrm{E}$
\end{tabular} & 3 & $\begin{array}{l}0.25 \mathrm{D}_{\mathrm{D}}, 0.5 \mathrm{D}_{\mathrm{D}} \\
1.0 \mathrm{D}_{\mathrm{D}}, 1.0 \mathrm{D}_{\mathrm{M}}\end{array}$ & - & $\mathrm{T}_{\mathrm{D}}^{(1)}$ \\
\hline 3 & $1.0 \mathrm{D}+0.5 \mathrm{~L}$ & 3 & $1.0 \mathrm{D}_{\mathrm{TM}}$ & - & $T_{D}{ }^{(1)}$ \\
\hline 4 & $1.0 \mathrm{D}+0.5 \mathrm{~L}$ & $30 \mathrm{~S}_{D 1} /\left(\mathrm{S}_{\mathrm{DS}} \mathrm{B}_{\mathrm{D}}\right) \geq 10^{(2)}$ & $1.0 \mathrm{D}_{\mathrm{TD}}$ & - & $T_{D}^{(1)}$ \\
\hline 5 & $\begin{array}{c}1.2 \mathrm{D}+1.0 \mathrm{E}+1.0 \mathrm{~L}+0.2 \mathrm{~S} \\
0.9 \mathrm{D}+1.0 \mathrm{E}^{(3)}\end{array}$ & 1 & $\begin{array}{c}0.25 \mathrm{D}_{\mathrm{D}}, 0.5 \mathrm{D}_{\mathrm{D}} \\
1.0 \mathrm{D}_{\mathrm{D}}, 1.0 \mathrm{D}_{\mathrm{M}}\end{array}$ & - & $\mathrm{T}_{\mathrm{D}}^{(1)}$ \\
\hline
\end{tabular}

Notes:

(1) Dynamic testing at the effective period TM are not required if dynamic prototype testing has already being performed on similar sized isolators, at similar loads, velocity, and displacement.

(2) $S_{D S}$ is the design $5 \%$ damped spectral acceleration value at $0.2 \mathrm{sec}$. Dynamic testing is performed in sets of 5 cycles.

TABLE 2.4: Sequence and cycles of prototype tests for ASCE 7-10

and

$$
\beta_{M}=\frac{\Sigma E_{M}}{2 \pi k_{M \max } D_{M}^{2}},
$$

$W$ - effective seismic weight of the structure above the isolation interface, $k_{D \min }$ - minimum effective stiffness of the isolation system at the design displacement in the horizontal direction under consideration, $k_{M m i n}-$ minimum effective stiffness of the isolation system at the maximum displacement in the horizontal direction under consideration, $\Sigma E_{D}$ and $\Sigma E_{M}$ are total amount of energy dissipated in the isolation system during a full cycle response at the design displacement $D_{D}$ and maximum displacement $D_{M}$, respectively, $y$ - the distance between the centers of rigidity of the isolation system and the element of interest measured perpendicular to to the direction of seismic loading under consideration, $e$ - the actual eccentricity measured in plan between the center of mass of the structure above the isolation interface and the center of rigidity of the isolation system, plus accidental eccentricity taken as $5 \%$ of the longest plan dimension of the structure perpendicular to the direction of force under consideration, $b$ - the shortest plan dimension of the structure measured perpendicular to $d$, and $d$ - the longest plan dimension of the structure. ASCE 7 (2010) allows further reduce $D_{T D}$ and $D_{T M}$ if the isolation system is shown by calculation to resist torsion. The summary of ASCE 7 (2010) Prototype tests is presented in Table 2.4.

Test specimen adequacy requirement presented in ASCE 7 (2010) have 5 parameters:

1. Force-deflection plots for all tests have a positive incremental force-resisting capacity, i.e. $\frac{d F}{d x}>0$.

2. For each increment of test displacement and for each vertical load case

- for each test specimen, the difference between the effective stiffness at each of the three cycles of test and the average value of effective stiffness is no greater than $15 \%$ 


\begin{tabular}{|c|c|c|}
\hline Req.\# & Reference Tests & Specimen adequacy requirement \\
\hline 1 & $1,2,3,4$ & $\mathrm{dF} / \mathrm{dx}>0$ \\
\hline 2 & 2 & $0.85 \leq \mathrm{k}_{\mathrm{eff},} /\left(\sum \mathrm{k}_{\mathrm{eff},} / \mathrm{N}\right) \leq 1.15$ \\
\hline 3 & 4 & $0.80 \leq \mathrm{k}_{\mathrm{eff},} / \mathrm{k}_{\mathrm{eff}, 1} \leq 1.20$ \\
\hline 4 & 4 & $0.80 \leq \varepsilon_{\mathrm{eff},} / \xi_{\mathrm{eff}, 1}$ \\
\hline 5 & 5 & Stability \\
\hline
\end{tabular}

Notes:

1. $F$ and $x$ are the lateral force and lateral deflection, respectively.

2. $k_{d}$ and $k_{\text {eff }}$ are post-yield and effective stiffness, respectively.

3. Subscripts ${ }_{1}$ and ${ }_{i}$ mean 1 st cycle, $i$-th cycle, average value for all cycles in a single test, and nominal value $N$ is the number of tested isolators of a common type.

TABLE 2.5: System adequacy requirements for ASCE7-10

- for each cycle of test, the difference between the effective stiffness of the two test specimens of a common type and size of the isolator unit and the average effective stiffness is no greater than $15 \%$

3. For each specimen there is no greater than $20 \%$ change in the initial effective stiffness over the cycles of test, i.e. $0.80 \leq k_{e f f, i} / k_{e f f, 1} \leq 1.20$.

4. For each specimen there is no greater than $20 \%$ decrease in the initial effective damping over the cycles of the test, i.e. $0.80 \leq \xi_{\text {eff }, i} / \xi_{\text {eff }, 1}$.

5. All specimens of vertical-load-carrying elements of the isolation system remain stable.

These requirements and corresponding tests are summarized in Table 2.5. It is important to note that current testing protocols were developed based on early experimental results of rubber bearings and can be improved by including adjustments that capture specific characteristics of friction isolation systems mentioned previously in detail in this paper such as the variability of the coefficient of friction $\mu$ due to increase in vertical loading, heating effects and varying velocity. 


\section{Chapter 3}

\section{Experimental Tests}

The equilibrium of forces acting on a slider in a concave sliding isolator results in a typical force distribution presented in Figure 3.1a. According to the simplified analytical model originally proposed by Zayas et al. (1990), the lateral force-displacement relationship based on the equilibrium is described as:

$$
F=\frac{W}{R} u+\operatorname{sign}(v) \mu W,
$$

where $W$ represents the applied vertical load simulating the weight of the superstructure, $u$ is the horizontal relative displacement between the slider and concave base, $v$ is the sliding velocity, $R$ is the radius of the concave surface, $\mu$ is the friction coefficient of the sliding system, and $\mathrm{F}$ is the applied horizontal force. If the applied vertical load $W$ and coefficient of friction $\mu$ remain constant, a theoretical shape of the forcedisplacement loop results in a parallelogram as presented in Figure 3.1b.

It can be noted from Figure 3.1b and Equation 3.1 that the externally applied horizontal force $F$ is partially resisted by the force $\mu W$ due to the frictional characteristics of the contact between slider and steel concave surface. The remaining force is resisted by the component associated with the "restoring stiffness":

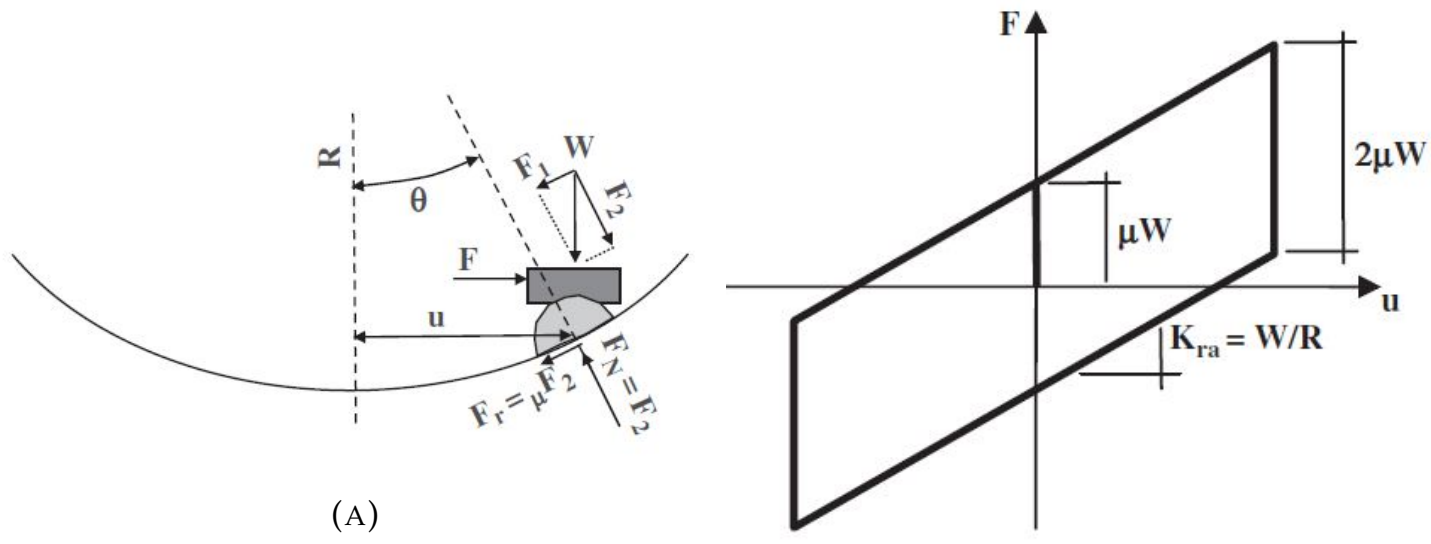

(B)

FIGURE 3.1: Theoretical behavior of concave sliding isolator: (A) force equilibrium and (B) force-displacement plot 


$$
K_{r a}=\frac{W}{R}
$$

Several models describing performance of sliding isolators have been presented in literature. Experimental model by Mokha et al. (1991) focused on bearings with high friction Techmet-B and low friction woven Teflon. The model accounted for the velocity effect and was able to reproduce results from unidirectional tests, but did not account for the reduction of friction coefficient with the increase of the number of cycles. Succeeding model was expanded to include high-velocity and bidirectional test results (Mokha et al., 1993). Similarly, a study by Constantinou et al. (1990) proposed experimental values for velocity, load, and breakaway effects, but could be further improved by inclusion of degradation of properties due to cycling. Recent models include Dolce et al. (2005) and Kumar et al. (2015), where authors proposed an experimental model for a single sliding concave friction isolator. The model accounts for the variations of friction coefficient $\mu$ as a function of sliding velocity, axial pressure, and temperature of the sliding surface. It is acknowledged that recording accurate measurements of temperature of the sliding surface while the slider is in motion is virtually impossible. In addition, the temperature of the sliding surface is highly affected by the ambient temperature, which makes the model calibration more complex.

\subsection{Experimental Data}

Experimental mono-directional tests were performed on double FPS using the procedures of ASCE 7 (2010). The raw data was provided by the experimental equipment. External sensors recorded the values of time, displacement, velocity, vertical and lateral forces in binary format. The data for each test was then stored on a computer hard drive. The users were allowed to determine the data storage location, output frequency, and starting delay. After extracting the raw output data from the setup equipment, the data was corrected to account for the shake table forces during the tests. As a general procedure adopted by the SRMD laboratory, the main objective of the data reduction process is to remove, from measured forces the components that are not directly applied to the test specimen; specifically the inertial and machine friction forces. This procedure required a theoretical and experimental phase of machine characterization and shakedown, mainly oriented to the assessment of the frictional and inertial characteristics of the system. Recorded forces are corrected based on the idealized horizontal equilibrium equation of the system:

Force readouts $=$ Shear across specimen + Inertial forces + Machine friction + Error,

where the Error term takes into account all the uncertainties related to the readout and correction process. The inertia forces could be estimated by means of the accelerometers mounted at the table top surface with longitudinal, lateral and vertical orientation. The horizontal mass was assessed during high-speed tests at assigned frequency and amplitude and calculated from actuator force-displacement hysteresis loops. Velocities 
and accelerations, used for correction purposes, can also be calculated as derivatives and double derivatives of the recorded displacements. When derivatives are used in the reduction process, in order to obtain smooth time histories of velocity and acceleration, a preliminary smoothing procedure is applied to raw displacement data using a Savitzky-Golay (polynomial) smoothing filter.

A significant number of experimental tests have been completed, in the machine shake-down phase, to identify the relationship between machine friction, generated at all the sliding interfaces, and vertical load amplitude, direction of motion, test velocity and ambient conditions. Tests performed on bare table and with a fifth bearing actuator, identical to the four vertical actuators installed under the table, permitted the creation of a database of results for machine friction assessment. In order to improve the assessment of the machine friction component, independent of the applied vertical load, "dry" runs were completed, without the test specimen, after each testing program, with displacement amplitudes and velocities identical to the ones applied to the isolators. The friction component obtained during these tests represents the most significant portion of the global machine friction force, due to the mechanism of friction development on the testing rig. The friction component due to the specific vertical load applied is added to the frictional forces recorded during the runs without the test specimen. With this procedure, the forces recorded during the actual tests, are corrected, point by point, by removal of the overall friction forces generated by the test equipment.

\subsection{Data Processing}

Corrected parameters were plotted against time to verify the accuracy of the performed tests. Typical graphs are presented below (Figure 3.2a,3.2b,3.2c,3.2d). It can be seen that for the same levels of longitudinal velocity, displacement and vertical load, the longitudinal force acting on the slider decreases with time, which indicates degradation of frictional properties. In addition, values of longitudinal force and vertical load were plotted against longitudinal displacement (Figure 3.2e, 3.2f). The reduction of area inside the force-displacement (hysteresis) loops correlates to the reduction of EDC - the amount of energy the system is able to dissipate per one cycle of motion.

After processing the recorded data, it was possible to determine the experimental values of friction coefficient $\mu$ and plot them against time (Figure 3.3a, 3.3b) and displacement (Figure 3.3c). The values of friction coefficient were computed by normalizing longitudinal force to vertical loading and subtracting a ratio of longitudinal displacement and the radius of curvature of the sliding surface:

$$
\mu=F_{\text {lat }} / W-D_{\text {lat }} / R
$$

where $F_{l a t}$ is longitudinal force, $W$ is vertical load, $D_{l a t}$ is longitudinal displacement, and $R$ is the effective radius of curvature of the isolator. This allowed to visually verify the behavior of double FPS. The reported vertical force represents the net force exerted by the four vertical actuators mounted under the platen and the eight outrigger actuators. Thus, the vertical force takes all weights and external vertical loads applied to the platen into account, and does not represent the vertical load applied to the test specimen. 


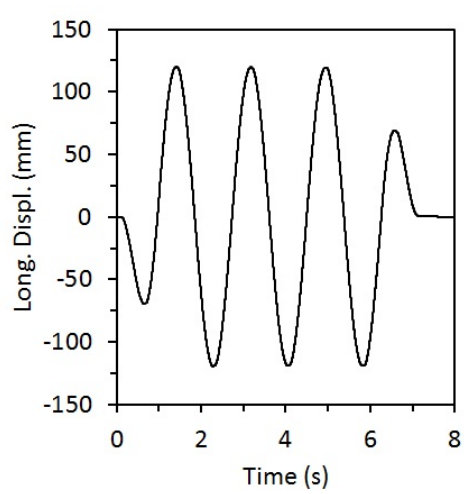

(A)

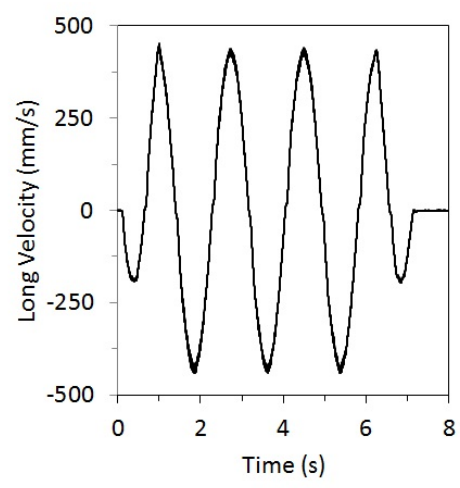

(D)

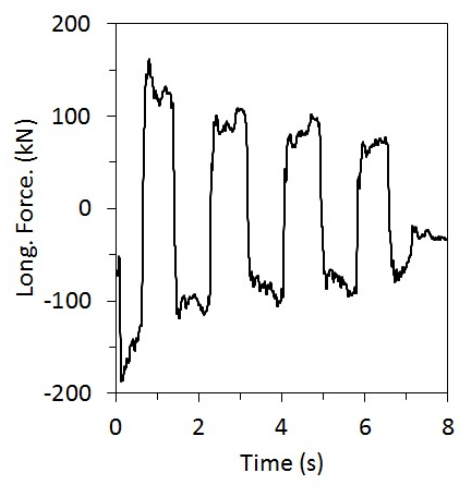

(B)

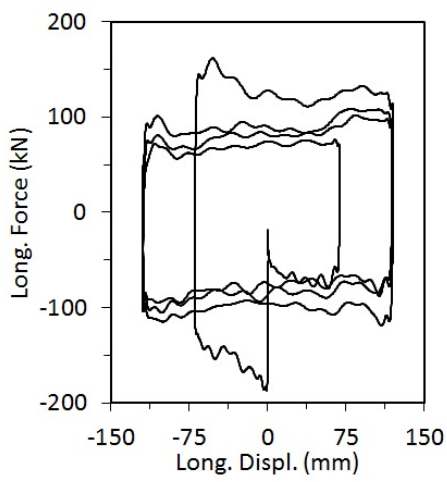

(E)

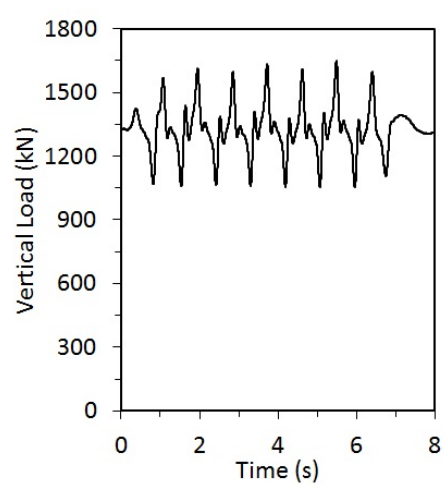

(C)

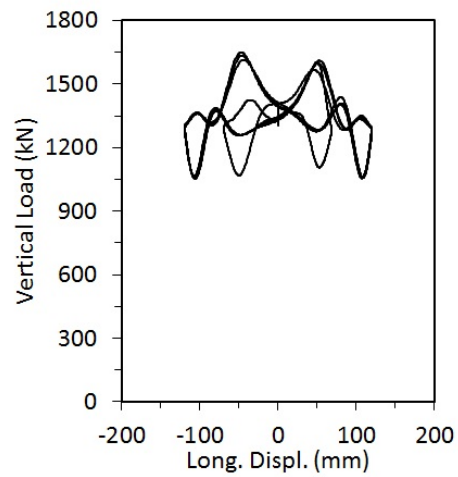

(F)

FIGURE 3.2: Typical test data

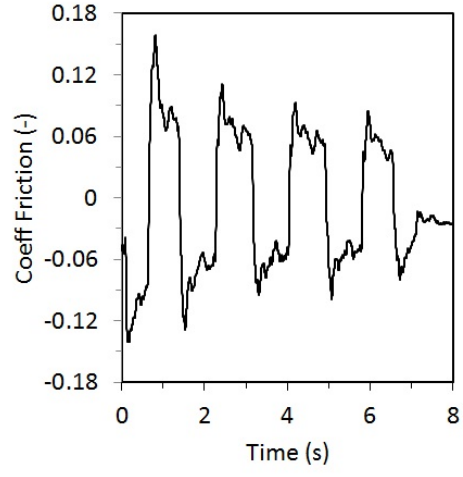

(A)

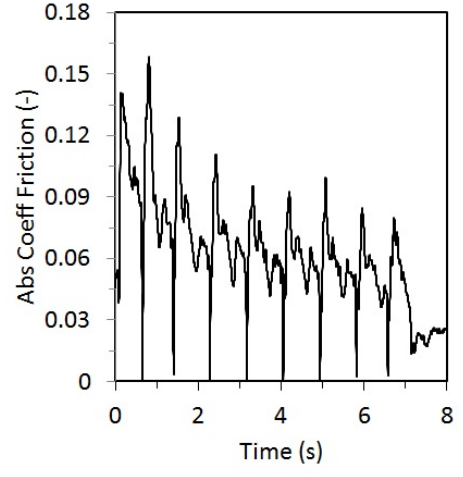

(B)

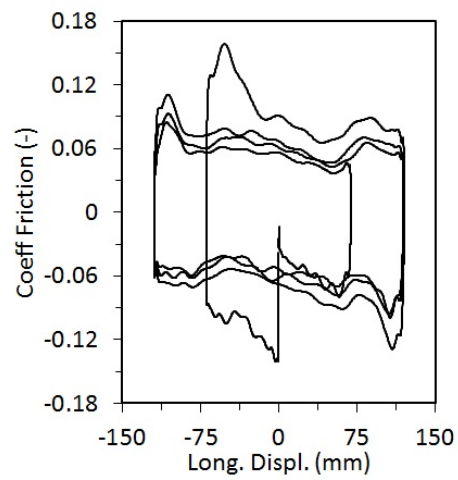

(C)

FIGURE 3.3: Typical plots obtained during the analysis 


\begin{tabular}{|c|c|c|c|c|c|c|}
\hline Test \# & Description & $\begin{array}{l}\text { Disp } \\
(\mathrm{mm})\end{array}$ & Freq $(\mathrm{Hz})$ & $\begin{array}{l}\text { Velocity } \\
(\mathrm{mm} / \mathrm{s})\end{array}$ & $\begin{array}{c}\text { Vert Load } \\
(\mathrm{kN})\end{array}$ & Cycles \\
\hline 1 & Test \#1 & 6 & 0.004 & 0.1 & 5643 & 1 \\
\hline 2 & \multirow{4}{*}{ Test $\# 2$} & 119 & 0.567 & 423 & 6800 & 3 \\
\hline 3 & & 238 & 0.283 & 423 & 6800 & 3 \\
\hline 4 & & 356 & 0.189 & 423 & 6800 & 3 \\
\hline 5 & & 460 & 0.146 & 423 & 6800 & 3 \\
\hline 6 & \multirow{4}{*}{ Test \#3 } & 119 & 0.567 & 423 & 6800 & 3 \\
\hline 7 & & 238 & 0.283 & 423 & 6800 & 3 \\
\hline 8 & & 356 & 0.189 & 423 & 6800 & 3 \\
\hline 9 & & 460 & 0.146 & 423 & 6800 & 3 \\
\hline 10 & Test \#4 & 460 & 0.146 & 423 & 5125 & 1 \\
\hline 11 & Test $\# 5$ & 460 & 0.146 & 423 & 7356 & 1 \\
\hline
\end{tabular}

TABLE 3.1: Summary of Prototype Testing

Overall, 7 double friction pendulum bearings were tested. Each bearing included two prototype isolators, $T 1$ and $T 2$, to ensure consistent performance, and 10-12 identical test runs were performed on each prototype. Table 3.1 presents a typical test summary of a single bearing. 


\section{Chapter 4}

\section{Model Calibration}

\subsection{Design Assumptions}

The experimental model presented in this paper focuses on the degradation of frictional properties in concave friction isolation systems due to the vertical load, velocity, and cycling effects. This study is based on several assumptions first proposed by Lomiento et al. (2013) during the calibration of a single concave sliding system's model:

1. The format of the functions of vertical load effect $f_{W}(W)$, velocity effect $f_{v}(v)$, and cycling effect $f_{c}(c)$ was obtained from the calibration of single sliding concave isolator. This reveals an underlying assumption that the behavior of a double sliding pendulum system is governed by the functions of the same format.

2. All of the aforementioned effects, namely $f_{W}(W), f_{v}(v), f_{c}(c)$, are independent from each other. While the assumption cannot be experimentally verified to be correct at this stage, models based on it have shown agreement with experimental results.

3. The heat distribution in double friction pendulum devices is based on single sliding pendulum system as presented later in this paper (Figure 4.10). This is a simplified approach as the double sliding pendulum system is an elaborate device with complex heat distribution parameters.

\subsection{Vertical Load Effect}

Experiments have indicated the degradation of frictional properties of an isolator with the increase of the applied vertical load. When the vertical load $W$ is increased, the coefficient of friction $\mu$ decreases; however, the friction force $f=\mu W$ increases due to the fact that the increase in $W$ has a greater effect than the decrease in $\mu$. This overall increase in the friction force $f$ corresponds to a greater amount of energy dissipated by the isolator per one cycle of motion (EDC). The increase in EDC can be represented as the increase in the area inside the hysteresis diagram with the increase in $W$ (Figure 4.1). To account for the variations in friction coefficient $\mu$, an exponential function characterizing vertical load effect $\left(f_{W}\right)$

$$
f_{W}(W)=\mu_{s 0} e^{-W / W_{r e f}}
$$

where $\mu_{s 0}$ represents the theoretical slow-motion coefficient of friction under no vertical 


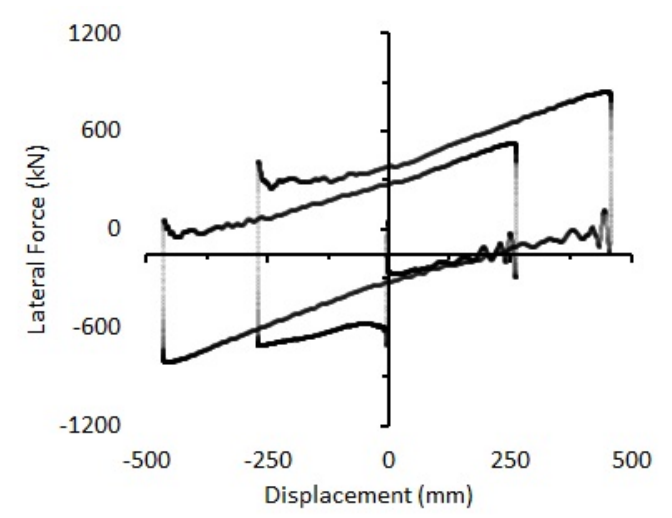

(A) $\mathrm{W}=5125 \mathrm{kN}$

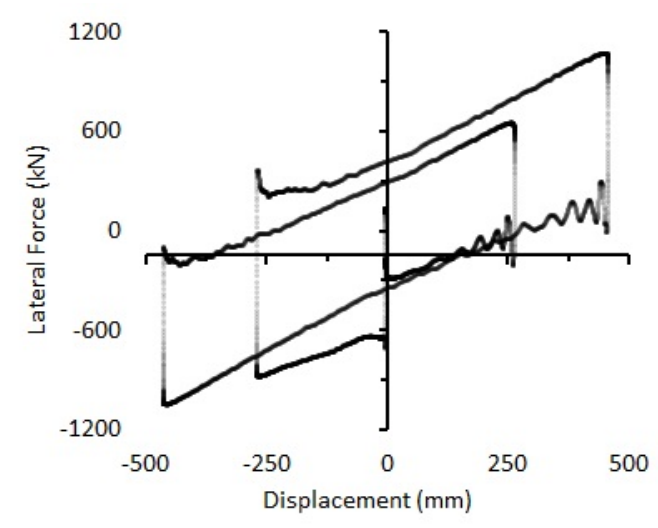

(в) $\mathrm{W}=7356 \mathrm{kN}$

FIGURE 4.1: Hysteresis plots for bearing 16 isolator under different vertical loads

load, $\mathrm{W}$ is the applied vertical load, and $W_{\text {ref }}$ is a load reference value, was proposed. The exponential format of the load effect function was selected based on the experimental evidence from the single pendulum isolator (Lomiento et al., 2013). To determine constant coefficients $\mu_{s 0}$ and $W_{r e f}$, it was necessary to minimize the influence of breakaway, cycling $\left(f_{c}\right)$, and velocity $\left(f_{v}\right)$ effects on friction coefficient $(\mu)$. Several trials were performed to achieve this goal. During the first calibration attempt, the data was extracted in the beginning of motion of the slider (Figure 4.2a). The following precautions were taken:

1. to neglect the contribution of velocity effect $\left(f_{v}\right)$, the data was extracted from regions of low velocity, i.e. $5 \mathrm{~mm} / \mathrm{s}<\mathrm{V}<10 \mathrm{~mm} / \mathrm{s}$;

2. to minimize the heat due to cycling effect $\left(f_{c}\right)$, only the data from the entrance loop (first loop of the hysteresis diagram) was used.

During the entrance loop, the slider is on its first cycle of motion; thus, the slider moves over a cold virgin surface and not absorbing any heat. On the first trial, bearing 16 isolator (brg16) was used.

As expected, after reviewing the experimental model resulting from the first trial, it was evident that the proposed approach was deficient as the function could not accurately capture the variations of the coefficient of friction $(\mu)$ due to changes in vertical load (W). After carefully examining the data and hysteresis plots, it was noted that one of the possible reasons for inaccuracy could be the breakaway effect. The extracted data was affected by the transition from static friction to kinetic. Also, low-velocity data is affected by electrical noise - undesired distortion of data due to random voltage fluctuations in equipment's electrical circuits, resulting in the theoretical region of zero velocity.

Second trial focused on the data within the entrance loop and same velocity values as used during the first trial $(5 \mathrm{~mm} / \mathrm{s}<\mathrm{V}<10 \mathrm{~mm} / \mathrm{s})$, but larger displacements to minimize the breakaway effect (Figure 4.2b). The data was extracted from time regions when the slider was coming to a stop near the maximum displacement from the center of the sliding surface. Separate models for bearings 16, 17 and 18 were developed. Values of $\mu$ and $W_{\text {ref }}$ were derived for each test and the combined model. 


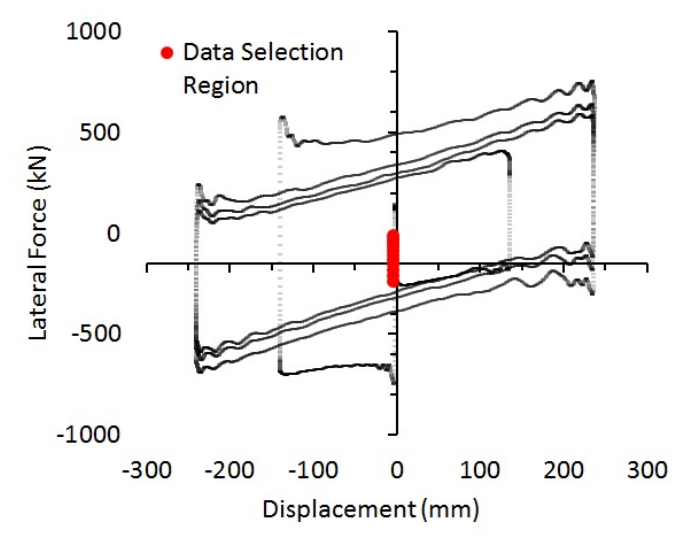

(A) Trial 1

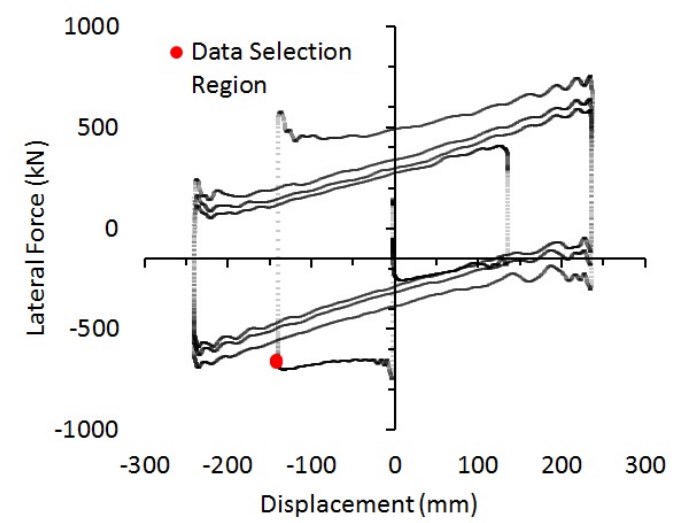

(B) Trial 2

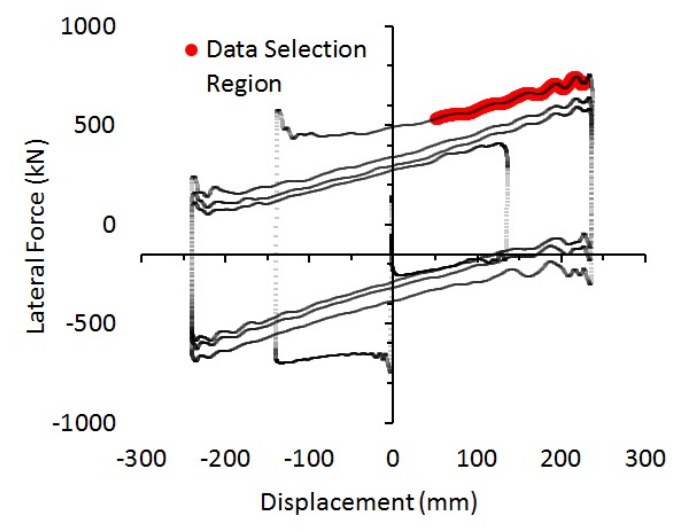

(C) Trial 3

FIGURE 4.2: Data selection regions

Evaluation of the models from Trial 2 concluded that the models offered little to no improvement over the previous trial. Possible explanation is that when the isolator comes to a stop, i.e. when velocity data are close to zero, the isolator reverses its motion. At the motion reversal, the data correction may significantly influence the data, as during the correction process the theoretically sudden reversal of motion converted to a more gradual transition. It is important to note that data correction plays a crucial role in these errors. For the next trial data for bearings 16, 17 and 18 was used. For Trial 3 the data was selected such that

1. the reversal of the direction of motion of the slider had already occurred;

2. slider passed the center of the base concave surface and was moving on the cold virgin surface;

3. the displacement values were selected to be greater than $50 \mathrm{~mm}$ to minimize the heat from the center of the concave sliding surface due to cycling effect;

4. velocity values were chosen to be greater than $100 \mathrm{~mm} / \mathrm{s}$. It was decided to use higher velocity data and account for the contribution of the velocity effect later (Figure 4.2c). 


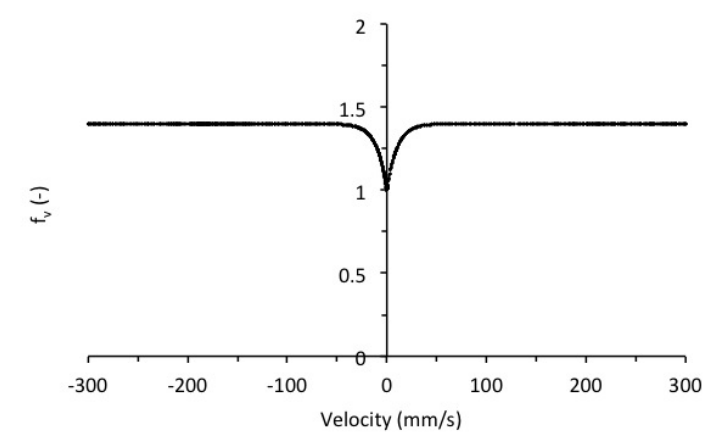

FIGURE 4.3: Velocity effect function

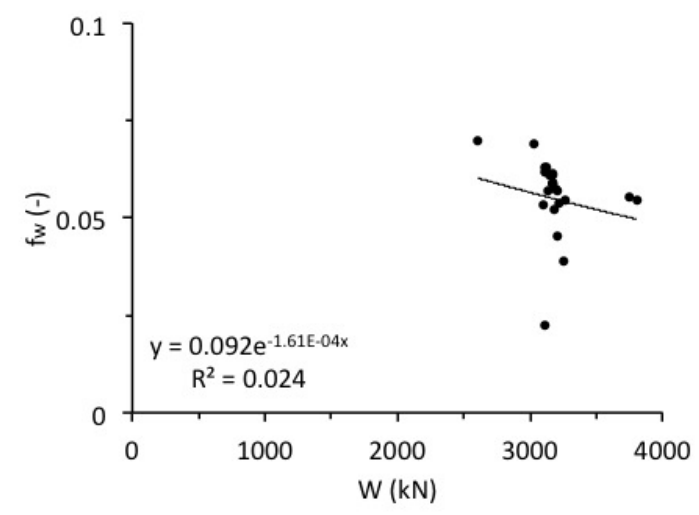

(A) Displacement $>50 \mathrm{~mm}$

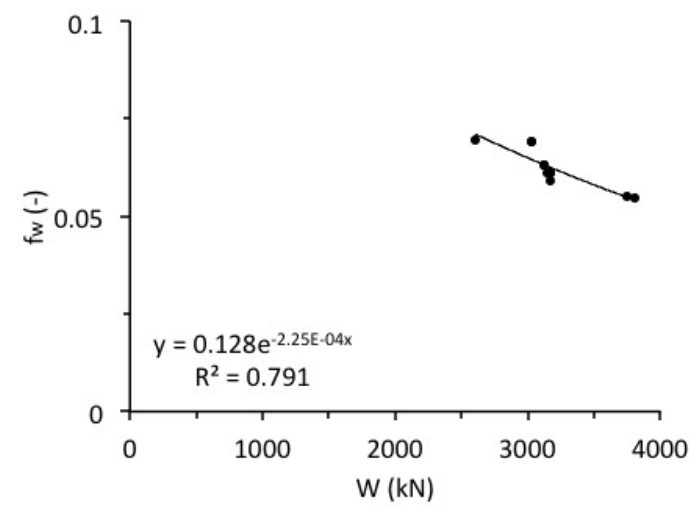

(B) Displacement $>300 \mathrm{~mm}$

FIGURE 4.4: Data outliers for bearing 18 isolator

In addition, all tests (brg16t1, brg16t2, brg17t1, brg17t2, brg18t1, and brg18t2) were analyzed in two stages: first, the data for each run was processed, and the experimental model was proposed. Second, same analysis was performed with the average values of lateral force and the friction coefficient $\mu$. The function of $f_{v}$ approaches a constant value of 1.4 for velocities higher than approximately $50 \mathrm{~mm} / \mathrm{s}$, thus, allowing to account for a constant adjustment later (Figure 4.3). The results of individual runs were compared with the averages. During the examination of data, it was noted that some of the outliers on the $\mu$ vs W plots can be eliminated if the value of the displacement is increased to approximately $300 \mathrm{~mm}$ (Figure 4.4).

As a result of using higher displacement data, the $R^{2}$ factor was significantly increased. It is important to note that when the slider passes over the center of the concave sliding surface after the reversal of motion, the center of the sliding surface has a greater temperature than the surrounding surface areas. Thus, increased displacement causes the reduction of heat experienced by the slider. At the final steps of the calibration, the $f_{W}$ function was reduced by a factor of 1.4 to account for the velocity effect. Calibration with the increased displacement values and adjustment for high velocity led to improved results and $R^{2}$ factor within the 0.57-0.95 range. 


\subsection{Velocity Effect}

The increase of the friction coefficient $\mu$ with increasing velocity is well-known and discussed extensively in literature (Constantinou et al., 1990; Mokha et al., 1991; Bondonet and Filiatrault, 1997; Dolce et al., 2005). These experimental studies, however, rarely extend to high velocity levels and, more importantly, can be further expanded to include double FPS devices. Lomiento et al. (2013) presented a model that included the relationship between the friction coefficient $\mu$ and sliding velocity for single concave sliding isolator devices (Figure 4.5). Dolce et al. (2005) reported on variations of friction coefficient with sliding velocity for cases with different pressure and ambient temperature (Figure 4.6). The study indicated that the coefficient of friction increased rapidly with velocity up to a velocity value of $150 \mathrm{~mm} / \mathrm{s}$, and then approached a constant value. This behavior was consistent regardless of ambient air temperature and bearing pressure on the slider. Similar dependency was observed during the testing of double FPS devices (Figure 4.7a). The sudden increase of friction coefficient at the regions of zero velocity is due to breakaway effect occurring at each reversal of motion.

In order to describe the relationship between the friction coefficient $\mu$ and sliding velocity, a function

$$
f_{v}(v)=\gamma+(1-\gamma) e^{-|v| / v_{r e f}}
$$

where $v$ is the sliding velocity, $v_{r e f}$ is a reference velocity that characterizes the variation rate, and $\gamma \geq 1$ is the ratio between the fast-motion and the slow-motion coefficient of friction, was used.

This function was previously used to describe the behavior of single FPS and proved a satisfactory agreement with the experimental data (Lomiento et al., 2013)). In order to accurately predict the behavior of the double FPS, constant coefficients $\gamma$ and $v_{r e f}$ had to be determined. For the purposes of this analysis, the data were chosen such that the reversal of motion of the isolator had already occurred, and the slider had passed the center of the concave surface. Similarly to the vertical load effect calibration (Figure
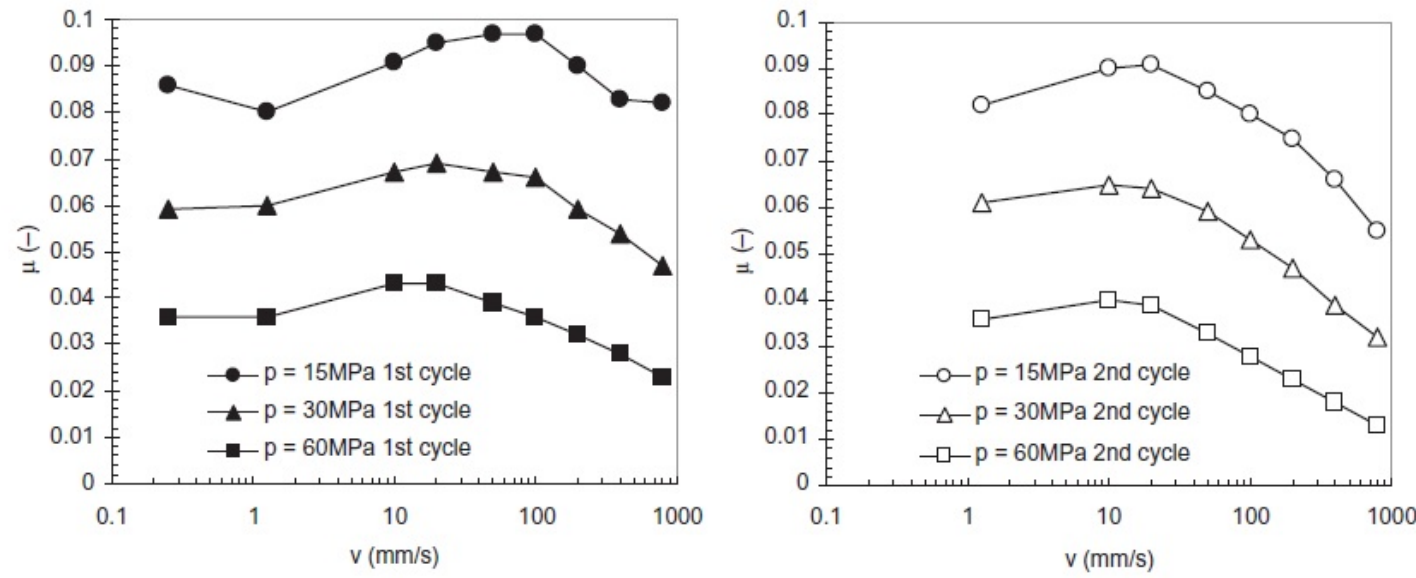

FIGURE 4.5: Experimental values of the friction coefficient $\mu$ vs. velocity presented by Lomiento et al. (2013). 


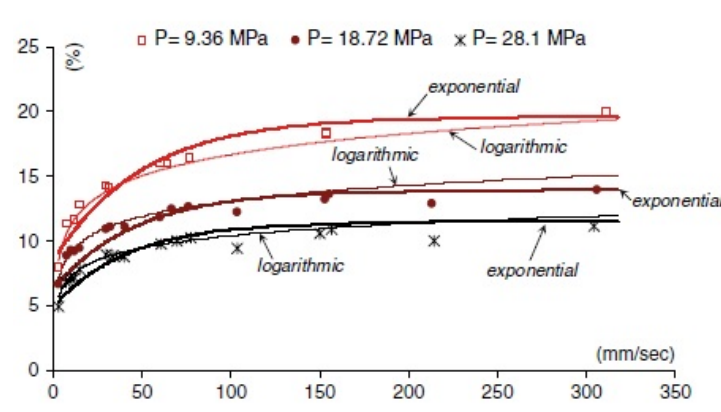

(A)

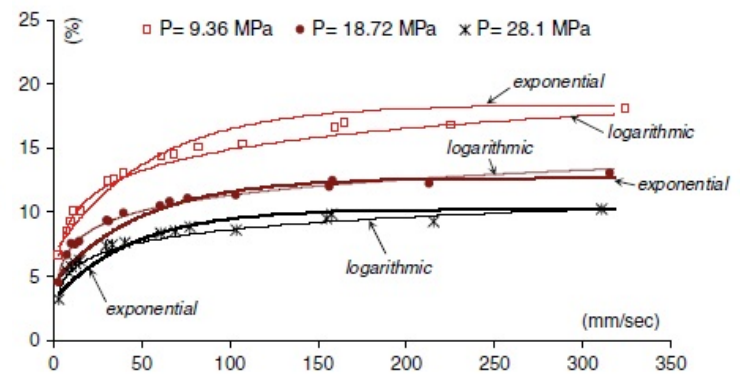

(B)

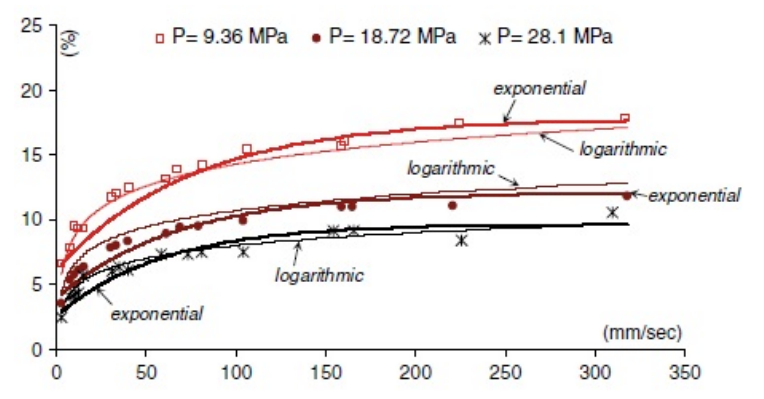

(C)

FIGURE 4.6: Variation of the friction coefficient with sliding velocity, air temperature and bearing pressure, for non-lubricated interfaces. Comparison between analytical laws and experimental results. Air temperature equal to: (A) $-10^{\circ} \mathrm{C}$, (B) $20^{\circ} \mathrm{C}$, and (C) $50^{\circ} \mathrm{C}$ (Dolce et al., 2005).

4.2c), this allowed to ensure that the slider was moving over the cold virgin surface. The displacement values were chosen to be greater than $150 \mathrm{~mm}$; thus, keeping the slider away from the center of the concave sliding surface where the most heat is expected. Velocity was selected to be greater than $10 \mathrm{~mm} / \mathrm{s}$ which allowed to capture both highand low-velocity behavior.

The analysis included the results from calibrations of vertical load and cycling effects to account for variations of friction coefficient with changes in vertical loading and increase of number of cycles, respectively. At this stage the preliminary vertical load effect function $f_{W}$ had been previously developed and was implemented into the calibration of velocity effect function $f_{v}$. Cycling effect function $f_{c}$ was assigned an assumed value obtained from a study on single FPS based on later-defined coefficients $c_{\text {ref }}$ and $\beta$ equal to 1900 and 0.5 , respectively. All three effects were combined into a single model to define the velocity effect function $f_{v}$. A regression analysis was performed on each FPS separately in order to minimize the influence of size and geometry variations among the bearings. Values of $\gamma$ and $v_{\text {ref }}$ were obtained. Figure $4.7 \mathrm{~b}$ presents the comparison of the velocity effect observed during the testing of double FPS vs the proposed model. The plot only focuses on the areas of the experimental graph not affected by breakaway effect. In both, the test data and the proposed model, the increase in friction coefficient with greater velocities is evident. 


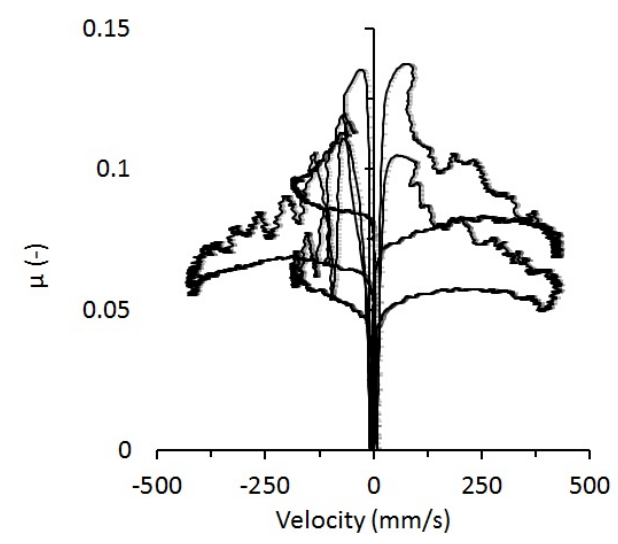

(A)

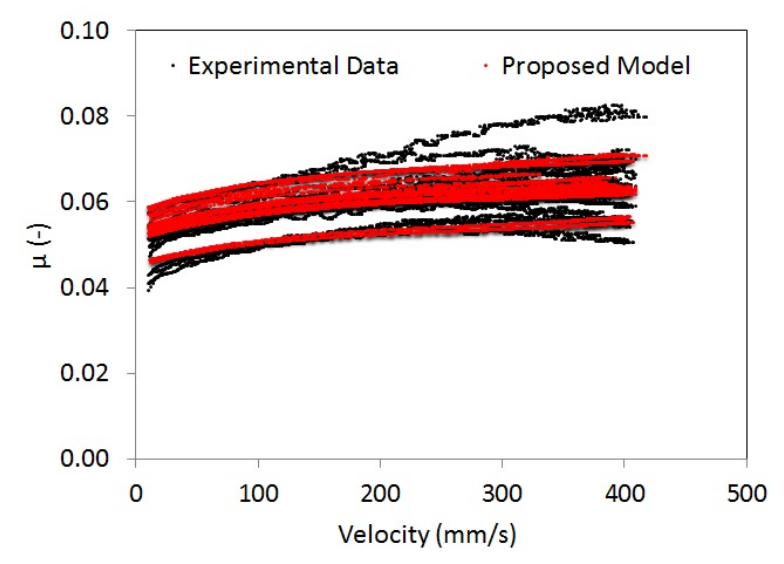

(B)

FIGURE 4.7: (A) Experimental variation of friction vs velocity, (B) Comparison of experimental data and proposed model during velocity effect calibration

\subsection{Cycling Effect}

Degradation of frictional material properties due to heating effects is well-known and has been extensively investigated. Previously proposed models mainly focused on the effects of ambient temperature on the behavior of sliding FPS. A study by Mokha et al. (1991) reported a decrease in kinetic coefficient of friction for PTFE-steel devices, but have not further investigated the phenomenon. According to thermodynamic principles, the degradation of frictional characteristics occurs due to reduction in hardness of the thin surface layer due to frictional heating (Lomiento et al., 2013). Chang et al. (1990) proposed a model for PTFE sliding isolators that included temperature variations at the sliding interface (Figure4.8). Lomiento et al. (2013) explained the mechanism and distribution of heat over the sliding surface and developed a model for a single FPS (Figure 4.9). The cycling effect function was based on a single FPS device and was presented in the following format:

$$
f_{c}(c)=e^{-\left(c / c_{r e f}\right)^{\beta}}
$$

where

$$
c(t)=\frac{2}{a \pi^{2} A^{2}} \int_{t_{0}}^{t} W v^{2} d t
$$

$c_{\text {ref }}$ represents the degradation rate of the friction coefficient with the cycling variable (smaller $c_{r e f}$ means faster degradation), the exponent $\beta$ controls the shape of the function, $a$ is the radius of the slider projection over the horizontal plane, $A$ - radius of the projection of the sliding surface over the horizontal plane, $W$ - vertical load, $v$ - instant sliding velocity, and $t_{0}$ and $t$ represent the beginning and end of the time interval under consideration. The function $c(t)$ represents the temperature effects over the sliding surface and implies the hypothesis of uniform distribution of the heat flux on the sliding surfaces (Lomiento et al., 2013). This assumption disregards the existence of higher heat fluxes in the areas interested by more frequent sliding activity. Figure 4.10 illustrates 


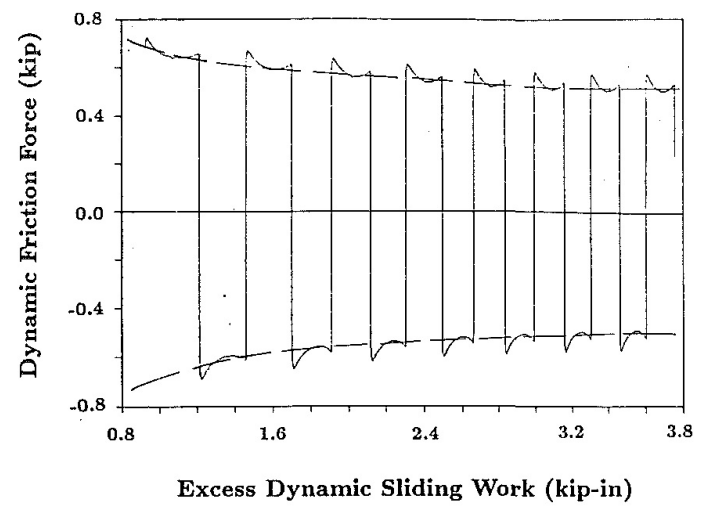

FIGURE 4.8: Degradation of frictional properties with increase in sliding work (Chang et al., 1990).

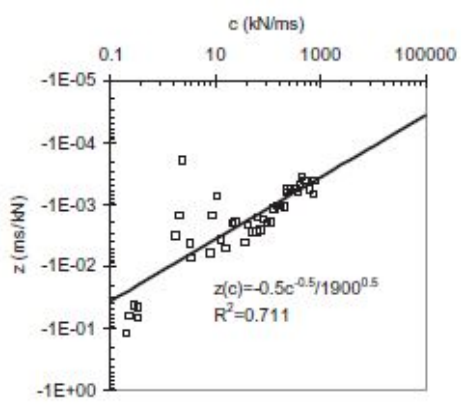

(a)

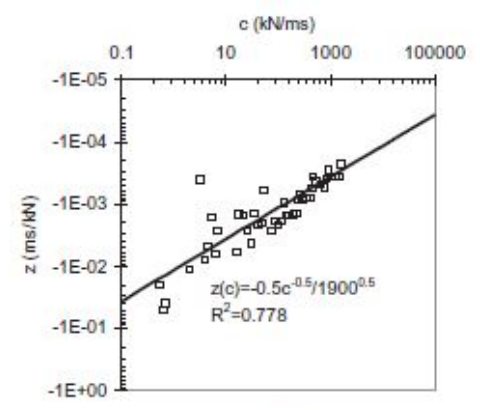

(b)

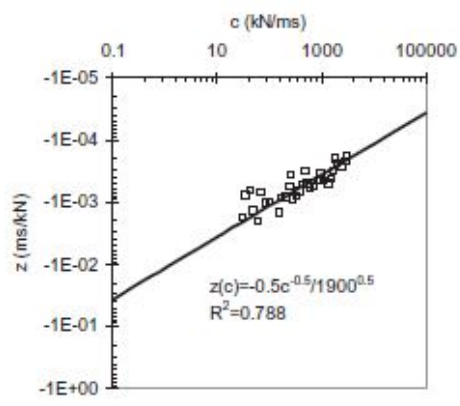

(c)

FIGURE 4.9: Correlation of function $z(c)$ with the experimental values of the cycling variable $c$ : (a) $p=15 \mathrm{MPa}$, (b) $p=30 \mathrm{MPa}$, (c) $p=60 \mathrm{MPa}$ presented by Lomiento et al. (2013).
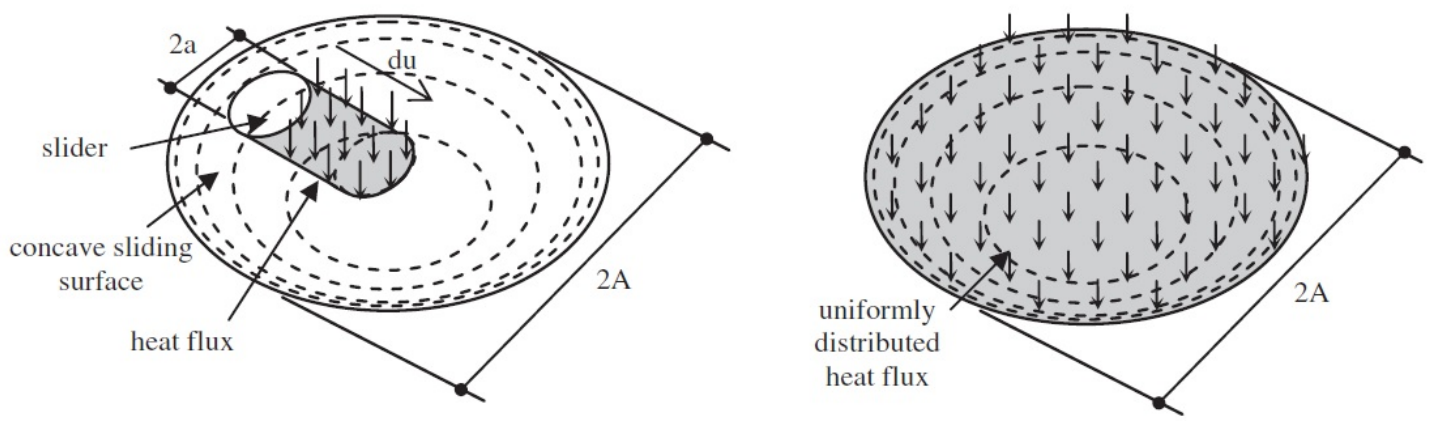

FIGURE 4.10: Actual and equivalent uniformly distributed heat flux in the single concave sliding isolator in the time interval $d t$. (Lomiento et al., 2013). 
the differences between the actual and assumed equivalent heat distributions for a single sliding pendulum isolation system. The heat distribution in the double pendulum system differs from the heat distribution in the single pendulum system, as in the case with double double sliding pendulum the heat is distributed over both, top and bottom, concave sliding surfaces. To obtain constant coefficients $c_{r e f}$ and $\beta$ presented in the Equation 4.3 a regression analysis was performed. Initial attempt focused on all of the collected data for all bearings. The data were combined into one model and parameters of $c_{r e f}$ and $\beta$ were modified to obtain the highest $R^{2}$ value as well as a satisfactory agreement between the experimental data and the proposed model. First trial resulted in the coefficient of degradation rate $c_{r e f}$ being a value significantly lower than expected in comparison to the results obtained from single concave sliding system tests discussed in Lomiento et al. (2013).

\subsection{Combined Model}

Separate experimental models were developed for each double concave sliding bearing. Due to variations in size among isolator devices, functions of vertical load effect $f_{W}(W)$ and cycling effect $f_{c}(c)$ were bearing specific, i.e. each isolator had its own set of constant coefficients. The velocity effect function $f_{v}(v)$ is not influenced by the size of an isolator, which allowed to include data from all bearing for the calibration of velocity effect function. Once preliminary functions of vertical load, velocity, and cycling effects were determined, all three functions were combined into a single model to represent the friction coefficient $\mu$ :

$$
\mu(W, v, c)=f_{W}(W) \cdot f_{v}(v) \cdot f_{c}(c)=\mu_{s 0} e^{-W / W_{r e f}} \cdot\left[\gamma+(1-\gamma) e^{-|v| / v_{r e f}}\right] \cdot e^{-\left(c / c_{r e f}\right)^{\beta}}
$$

An important step was to validate the original assumptions made during the calibration of vertical load and velocity effects:

1. $\gamma_{\text {ref }}=1.4, f_{c}=1$ for $f_{W}$ calibration

2. $c_{\text {ref }}=1900, \beta=0.5$ for $f_{v}$ calibration

The coefficients of $c_{r e f}$ and $\beta$ obtained during the calibration of cycling effect were imported into velocity effect model. This allowed to derive new values for $\gamma$ and $v_{r e f}$ which then were plugged into cycling effect model to obtain the second-iteration values of $c_{r e f}$ and $\beta$. The $f_{W}$ function was adjusted to reflect the new value of $\gamma-$ a change that accounted for a vertical shift of the graph. This iterative approach was used, until convergence was achieved. Figures $4.11 \mathrm{a}$ and $4.11 \mathrm{~b}$ demonstrate the overlay of proposed model with experimental results. The poor agreement between the model and experimental data is caused by the fact that constants used for calibration were derived based on selected tests (i.e. the calibration of vertical load effect was based on high displacement tests) and not on all the data combined. To correct for this disagreement, the proposed model was further adjusted until it agreed with the test data. Figures 4.11c and 4.11d present a better agreement of test data with proposed model achieve by adjusting coefficients $\mu_{s, 0}, c_{r e f}$ and $\beta$. 

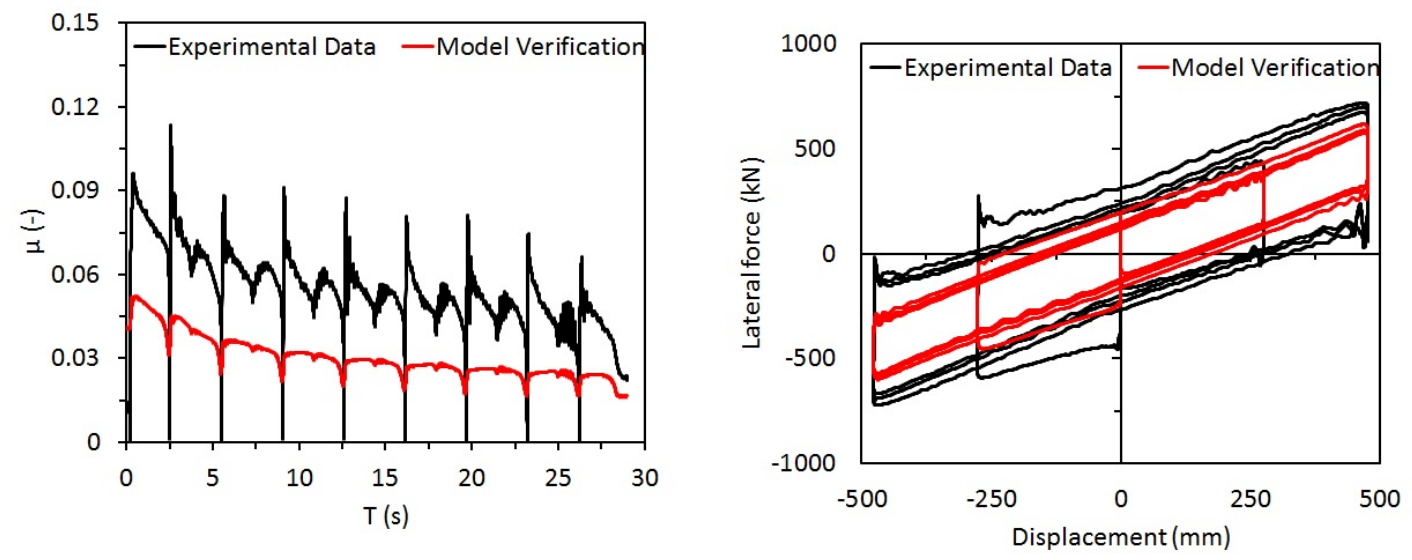

(A) Degradation of frictional properties with time before adjustment

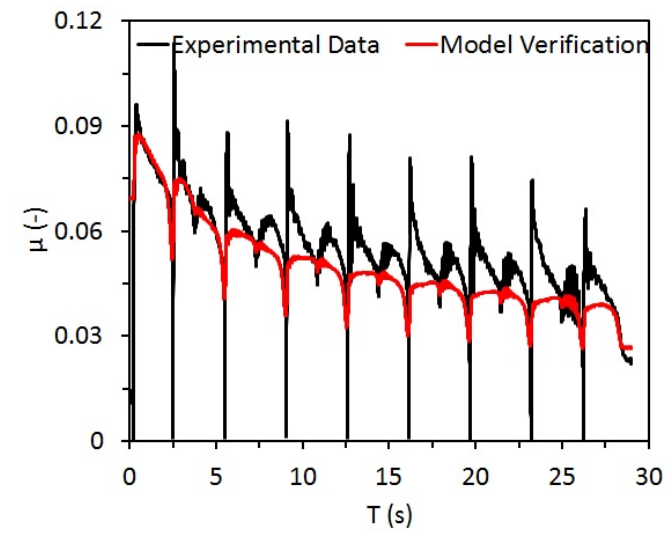

(B) Force-displacement plots before adjustment

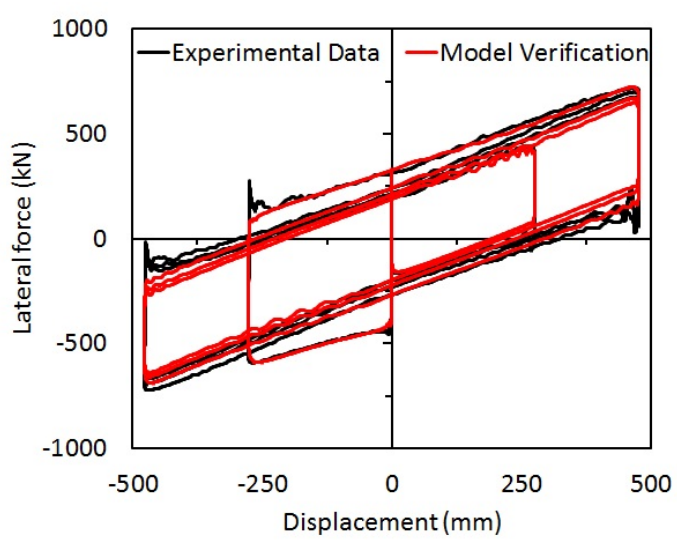

(C) Degradation of frictional properties with time after adjustment

(D) Force-displacement plots after adjustment

FIGURE 4.11: Comparison of experimental data and proposed model 


\section{Chapter 5}

\section{Model Validation}

\subsection{Investigation Results}

The iterative procedure and later adjustment described in Section 4.5 allowed to calibrate the parameters required for the proposed model (Table 5.1). Utilizing these parameters, hysteresis plots were developed for each isolator bearing. It is important to note that the proposed model was verified against the tests with displacements greater than $300 \mathrm{~mm}$ as this displacement value is more representative of the design conditions for seismic loads. A complete set of comparison plots of the experimental results and proposed model is presented in Appendix A.

\subsection{Discussion}

The obtained results indicate that the proposed model is capable of capturing the behavior of the double concave sliding isolators influenced by vertical load, velocity and cycling effects for mono-directional tests. The sudden increase of friction at each reversal of the direction of motion (i.e. breakaway effect) is well-depicted on Figures 5.1a, 5.1c, and 5.1e. In reality, this has little effect on the performance of the isolation device due to the fact that seismically induced motions are bi-directional without reversal of sliding motion. Thus, during an earthquake transition from static to dynamic friction is only observed at the beginning of the first cycle of motion. For this reason breakaway effect remained outside of the scope of this study and, therefore, was not included in the

\begin{tabular}{|c|c|c|c|c|c|c|c|}
\hline Parameter & \multicolumn{1}{c}{ brg16 } & \multicolumn{1}{c}{ brg17 } & \multicolumn{1}{l}{ brg18 } & \multicolumn{1}{c|}{ brg19 } & \multicolumn{1}{l|}{ brg20 } & \multicolumn{1}{c|}{ brg21 } & \multicolumn{1}{c|}{ brg22 } \\
\hline $\mathbf{R}(\mathbf{m m})$ & 5000 & 5000 & 5000 & 5000 & 3400 & 3400 & 3400 \\
\hline $\mathbf{a}(\mathbf{m m})$ & 323.1 & 423.4 & 323.1 & 423.4 & 518.7 & 423.9 & 328.4 \\
\hline $\mathbf{A}(\mathbf{m m})$ & 802.4 & 974.4 & 802.4 & 974.4 & 1020.7 & 907.6 & 774.8 \\
\hline $\boldsymbol{\mu}_{\mathbf{s , 0}}$ & 0.1597 & 0.1143 & 0.1180 & 0.2235 & 0.1702 & 0.1634 & 0.1541 \\
\hline $\mathbf{W}_{\text {ref }}(\mathbf{k N})$ & 6906 & 7443 & 4136 & 1415 & 7052 & 5400 & 3579 \\
\hline $\boldsymbol{\gamma}$ & 1.48 & 1.48 & 1.48 & 1.48 & 1.48 & 1.48 & 1.48 \\
\hline $\mathbf{V}_{\text {ref }}(\mathbf{m m} / \mathbf{s})$ & 50 & 50 & 50 & 50 & 50 & 50 & 50 \\
\hline $\mathbf{c}_{\text {ref }}(\mathbf{k N} / \mathbf{m m s})$ & 17.0 & 10.0 & 20.0 & 10.0 & 10.0 & 10.0 & 20.0 \\
\hline $\boldsymbol{\beta}$ & 0.400 & 0.400 & 0.250 & 0.150 & 0.300 & 0.300 & 0.250 \\
\hline $\mathbf{R}_{\text {ave }}^{2}$ & 0.540 & 0.552 & 0.507 & 0.367 & 0.561 & 0.576 & 0.494 \\
\hline
\end{tabular}

TABLE 5.1: Final values of constant coefficient and isolator parameters 


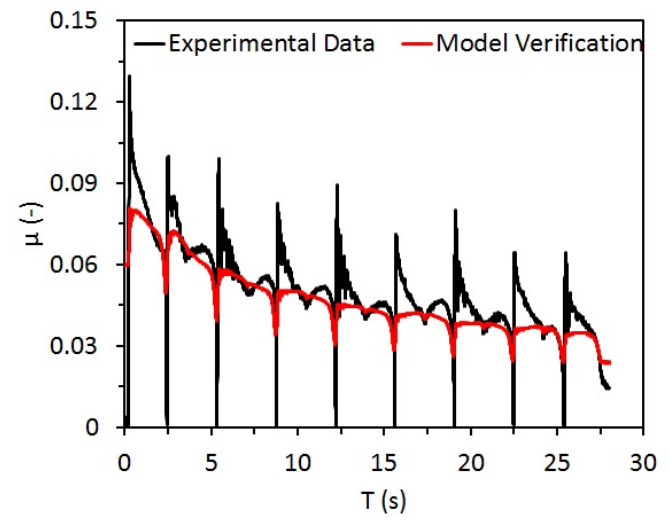

(A) brg16t1run9

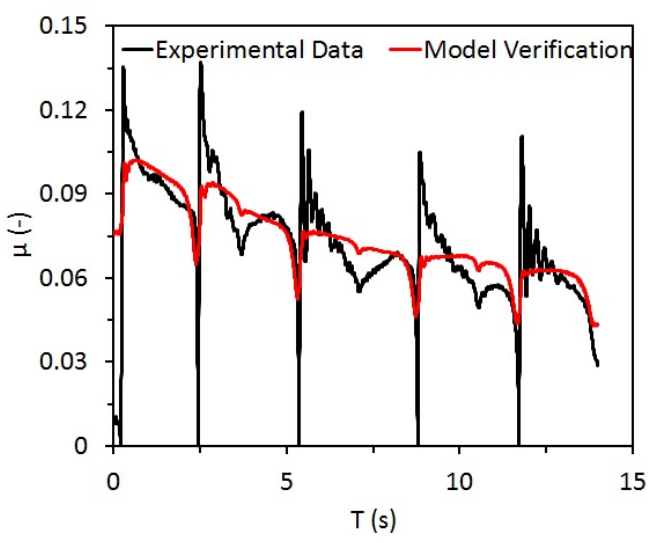

(C) brg16t1run10

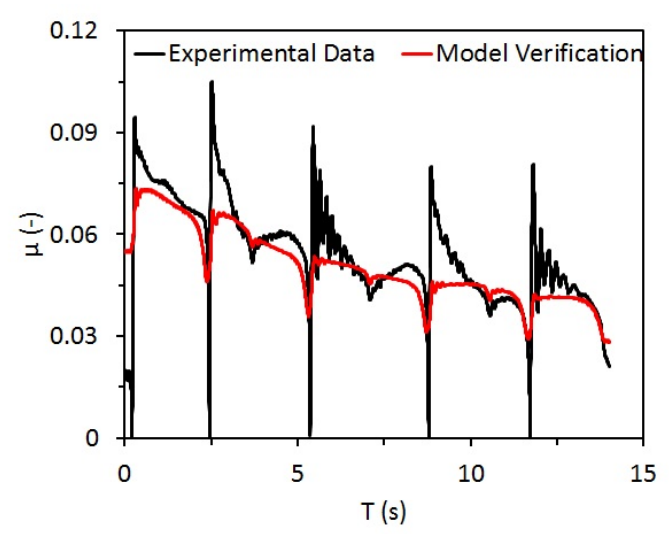

(E) brg16t1run11

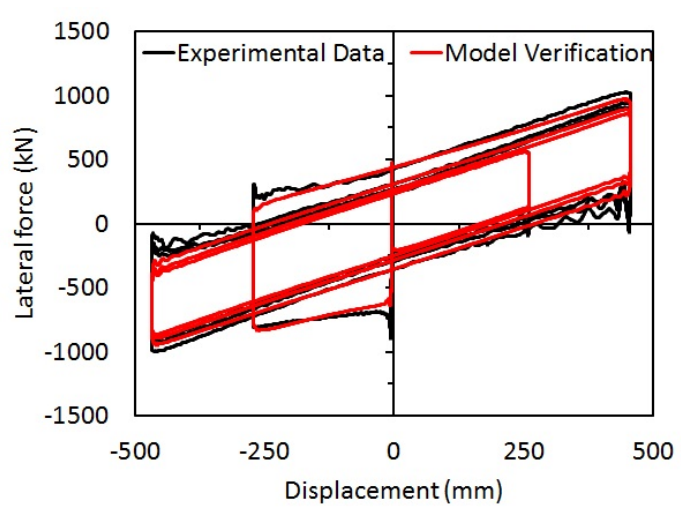

(в) brg16t1run9

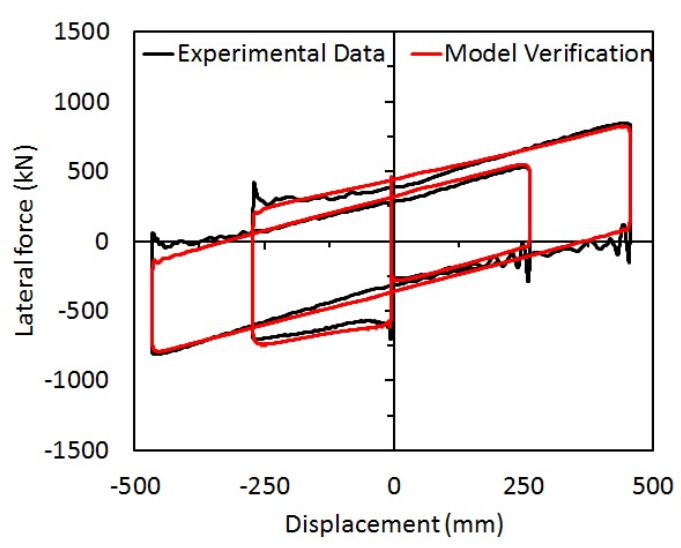

(D) brg16t1run10

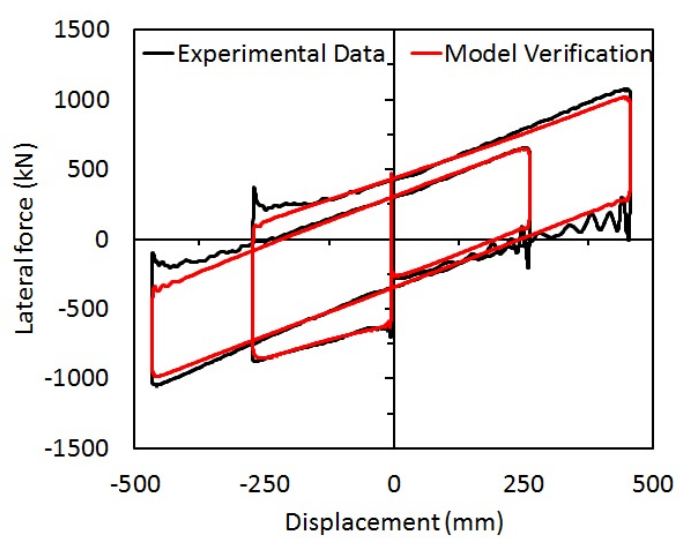

(F) brg16t1run11

FIGURE 5.1: Comparison of experimental data and proposed model for bearing 16 


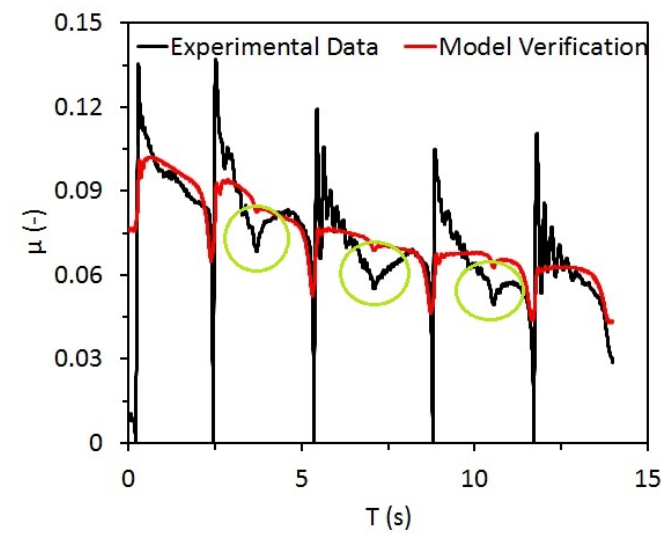

(A)

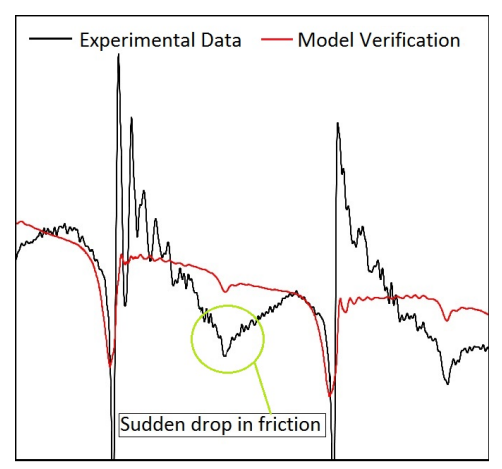

(B)

FIGURE 5.2: (A) A sudden drop in friction due to isolator geometry, and

(B) zoomed in look for bearing 16 run 10

model. The significance of this study is the ability of the model to closely simulate the experimental force-displacement plots - an important parameter in determining energy dissipation characteristics of a system under cyclic loading. Table 5.1 presents the $R^{2}$ values representing the correlation between the experimental results and the proposed model. The tabulated $R^{2}$ values are the averages of all the runs used to calibrate a particular bearing. The proposed model results in $R^{2}$ values of approximately 0.5 ; however, neglecting the breakaway effect will result in greater $R^{2}$ values. Comparison of the behavior of single and double FPS indicated that influence of vertical load and velocity effects is similar between the two systems, and constant coefficients $\mu_{s, 0}, W_{r e f}, \gamma$, and $V_{\text {ref }}$ for a single FPS model presented by Lomiento et al. (2013) are comparable to those discovered by this study. However, a large difference was noted during the comparison of cycling effect $f_{c}$ between two systems. The coefficients $\beta$ responsible for the shape of the function's curve is similar in value between single and double systems; however, the coefficient $c_{r e f}$ representing the rate of degradation of frictional properties of the material due to cycling is much smaller for a double pendulum system. For instance, typical value of $c_{r e f}$ for a single FPS is $1.9 \frac{\mathrm{kN}}{\mathrm{mms}}$, while values for a double FPS system range between 10 and $20 \frac{\mathrm{kN}}{\mathrm{mms}}$. A possible explanation of this is a different heat distribution pattern in a double pendulum isolator than a single pendulum model assumes (Figure 4.10). Lower $c_{r e f}$ in single pendulum system corresponds to a faster degradation rate of frictional properties in single friction isolation system. This can be caused by the fact that in the case with double sliding friction system, the heat is distributed over the two sliding surfaces, top and bottom, instead of just one surface in the case of a single FPS. This distribution possibly results in lower temperatures in each of the two surfaces. As a result, there is less degradation of frictional properties due to cycling. The presence of the slider between the two surfaces also affects heat distribution as some heat can probably be dissipated through the slider itself.

Another behavioral aspect that is unique to double FPS and was not present in a single FPS is a sudden drop in the magnitude of the friction coefficient $\mu$ when the slider passes over the center of the sliding surface. This drop is evident on Figure 5.2, but is not evident on hysteresis plots (Figures 5.1b, 5.1d, and 5.1f). This phenomenon is a result 


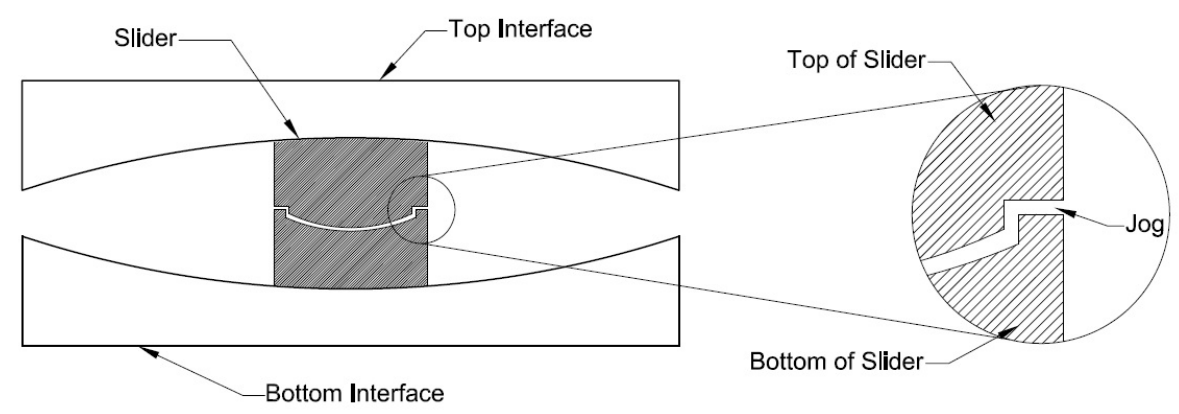

(A) Undeformed configuration

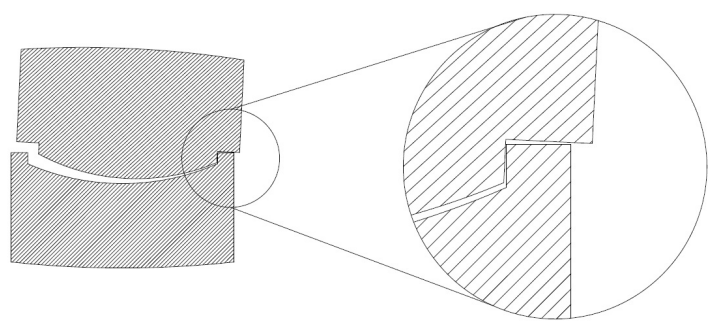

(B) Deformed configuration

FIGURE 5.3: Diagram of the slider illustrating the mid-joint

of the isolator's design: to account for rotation of the slider during the motion, and to keep the top and bottom surfaces of the device parallel, the slider has an adjustment mechanism - a sliding joint that allows a certain degree of rotation of the top slider's surface relatively to its bottom surface (Figure 5.3a). When the sliding joint is engaged, it creates a sudden drop in friction as seen on aforementioned figures. In these regions the proposed model is unable to fully capture the "drop" behavior and overestimates the experimental data.

The combined model presented in this paper includes all three frictional effects as mentioned previously, making it difficult to estimate the contribution of each single effect separately. Despite that, it can be concluded that based on model's prediction during one cycle of slider's motion, the increase in friction coefficient $\mu$ due to velocity effect is dominant over the reduction in $\mu$ due to generated heat. Figures 5.1a, 5.1c, and 5.1e present the proposed model suggests an increase in friction coefficient $\mu$ when the slider passes over the center of the sliding surface, a region of maximum velocity and heat. Since the increase in $\mu$ is present, the degradation of frictional properties due to heat is overcome by the increase of friction with the increase in sliding velocity.

Evidence from hysteresis plots (Figures 5.1b, 5.1d, and 5.1f) suggests that the proposed model underestimates the frictional force at the regions when the slider is coming to a stop. This is especially evident at the first cycle of motion, when the slider is moving over a cold "virgin" surface (Figures 5.5a, and 5.5b), and less so for a greater number of cycles (Figure 5.1b). 


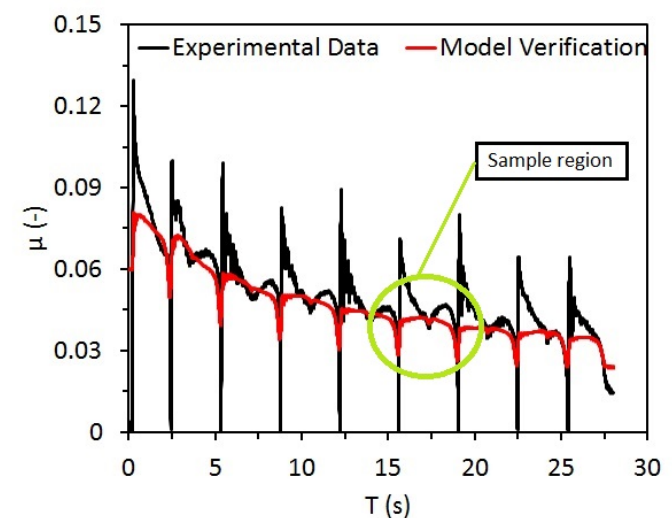

(A)

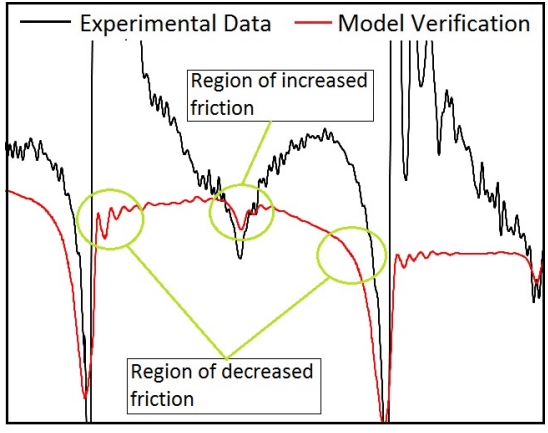

(B)

FIGURE 5.4: (A) Areas affected by the velocity and cycling effects, and (B) zoomed in look for bearing 16 run 9

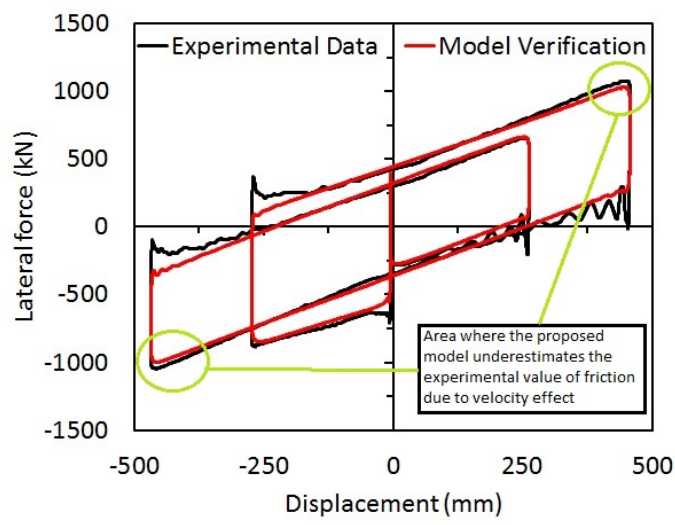

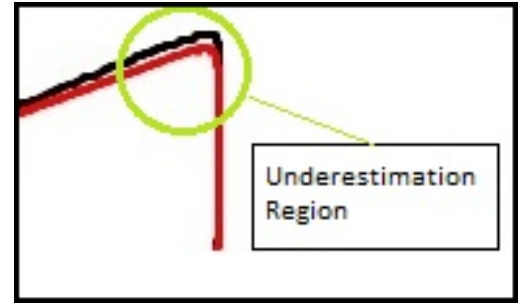

(B)

(A)

FIGURE 5.5: (A) Areas of hysteresis loop affected by the velocity effect and underestimated by the proposed model, and (B) zoomed in look for bearing 16 run 11

\subsubsection{Adequacy of Testing Protocols}

Isolator testing protocols presented in ASCE 7 (2010) can be improved to efficiently develop a model describing devices' behavior, which makes the use of this technology expensive, limited, and reserved for project-specific investigations. Over the course of this study it was demonstrated that a complex iterative procedure is required to describe double sliding pendulum system's behavior. Moreover, ASCE 7 (2010) utilizes testing protocols that were originally intended for rubber HDPE bearings and can be expanded to account for specifics of sliding concave isolators. Such newly discovered effects include variability of the coefficient of friction $\mu$ due to aforementioned effects $f_{W}(W), f_{c}(c), f_{v}(v)$ as well as breakaway effect. This fact leaves room for improvement of the testing procedures for all sliding concave systems (single, double, or triple).

Possible improvement over the currently accepted testing protocols is a series of 
tests targeting specific behavioral characteristics of friction isolation systems such as the vertical load, velocity and cycling effects. For instance, the vertical load effect can be addressed through a quasi-static test in which the velocity and cycling effects are negligible. Similarly, the contribution of the velocity effect can be accounted for through a velocity test with a quadratic displacement pattern. The maximum value of velocity should be kept low to reduce cycling effect. Finally, to address the cycling effect separately from other effects, a test with constant velocity and vertical load can be performed (Lomiento and Benzoni, 2017). 


\section{Chapter 6}

\section{Conclusion}

Full-scale mono-directional tests were performed on seven double sliding pendulum bearings in accordance with ASCE 7 (2010) testing protocols. An experimental model addressing the degradation of frictional properties in single concave sliding bearings developed by Lomiento et al. (2013) was applied to double concave sliding bearings. Friction coefficient $\mu$ was represented as a product of the vertical load effect $f_{W}(W)$, velocity effect $f_{v}(v)$, and cycling effect $f_{c}(c)$ functions that address the dependency of the friction coefficient $\mu$ on applied vertical load, sliding velocity and repetition of cycles, respectively. Each of the three independent functions included in the model was calibrated using test data to account for variations in friction coefficient $\mu$ in double pendulum systems. The proposed model was calibrated against the experimental data. Applicability of the model to the double pendulum bearing systems was discussed. Similarities and differences between the model by Lomiento et al. (2013) and the proposed model were evaluated.

Results suggest the degradation of frictional properties up to $50 \%$ of the original value in a matter of just several cycles. It was noted that cycling effect function $f_{c}$ differs between the models of single and double pendulum systems. A possible explanation is a different heat distribution in double versus single pendulum devices. In double pendulum isolators the heat is distributed to both top and bottom sliding surfaces affecting the sider in between. In single pendulum systems where only one sliding surface is present at the bottom, the heat is mainly distributed over one sliding surface. Other parameters discussed in this paper, namely vertical load and velocity effects, are comparable between the two systems. Experimental data also revealed that double pendulum isolators a sudden drop in friction occurs due to the design of the slider. The slider is constructed of top and bottom interfaces, which can move slightly relative to each other in order to keep top and bottom of the slider in contact with concave sliding surfaces. Breakaway effect was present during the tests, however, remained outside of the scope of this study as its presence is not anticipated during bidirectional motion typical to earthquakes.

Experimental tests performed in accordance with current testing protocols required a complex iterative procedure in order to address the individual contributions of the vertical load, velocity, and cycling effects on the friction coefficient. A simplified testing procedure focusing on vertical load, velocity and cycling effects separately is necessary which can be achieved by specifically targeting the aforementioned effects during testing. 
Appendix A

Model Verification Plots 


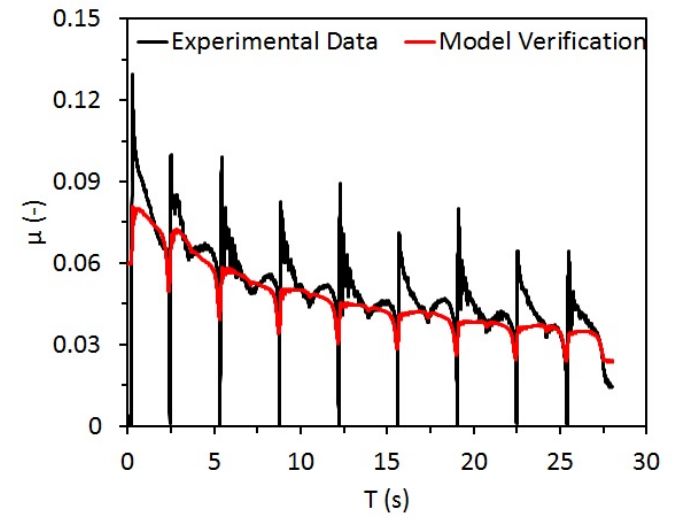

(A) brg16t1run9

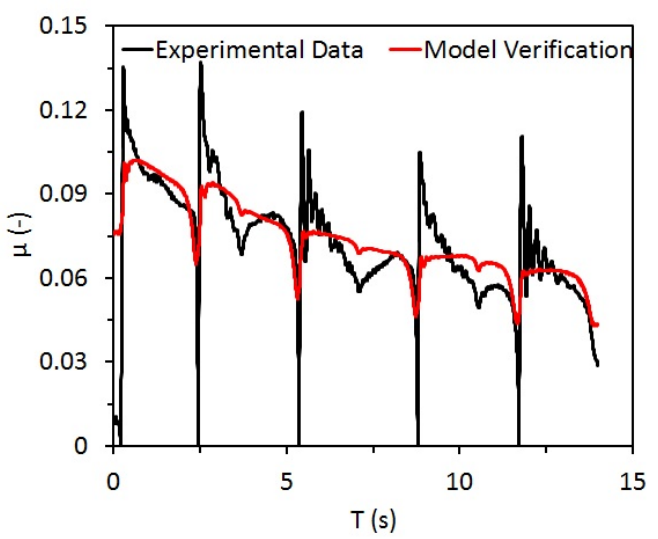

(C) brg16t1run10

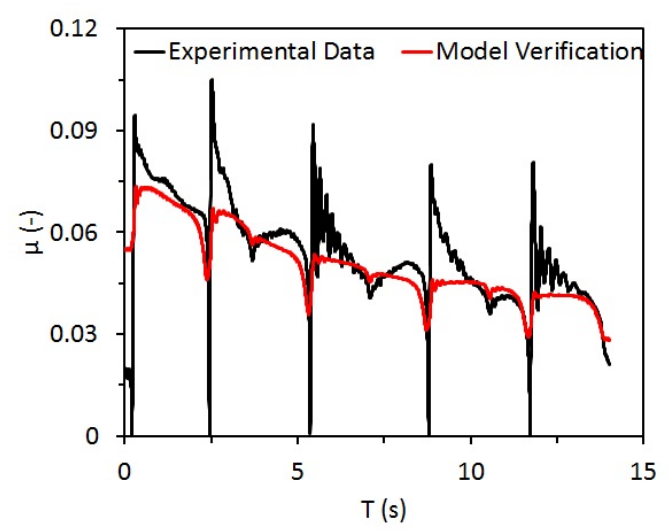

(E) brg16t1run11

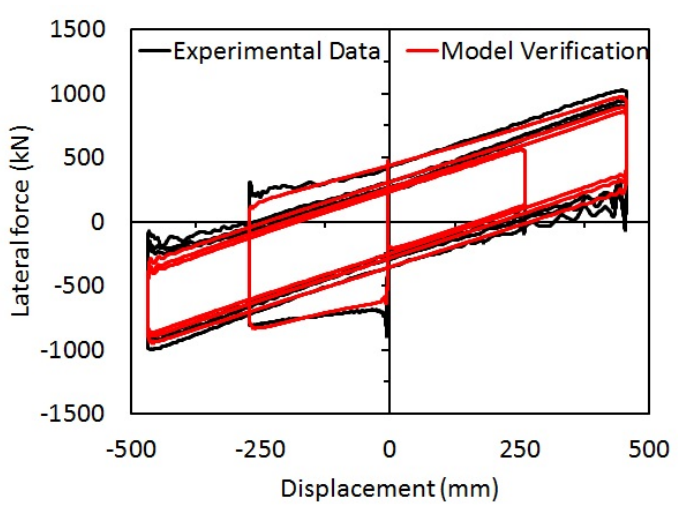

(B) brg16t1run9

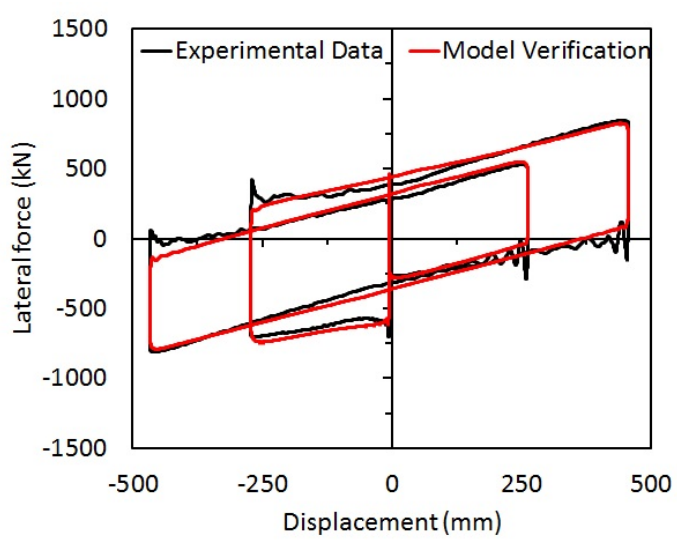

(D) brg16t1run10

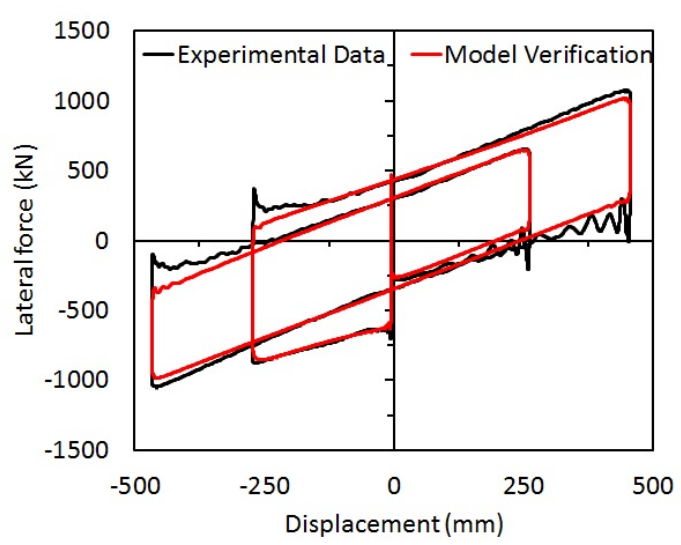

(F) brg16t1run11

FIGURE A.1: Bearing 16 


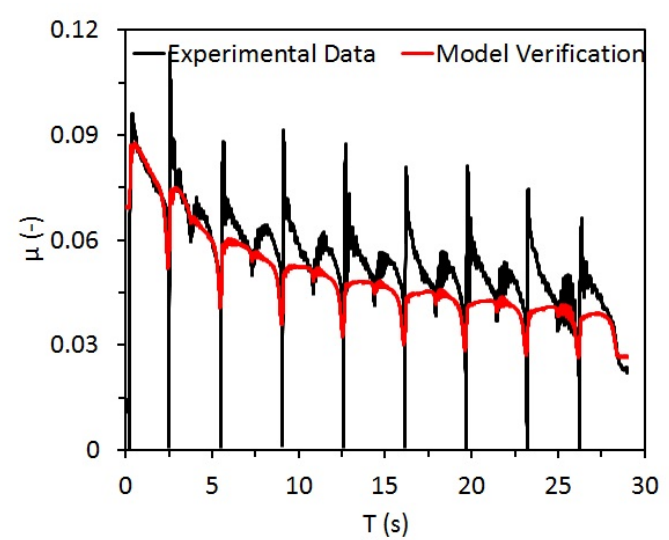

(A) brg17t1run9

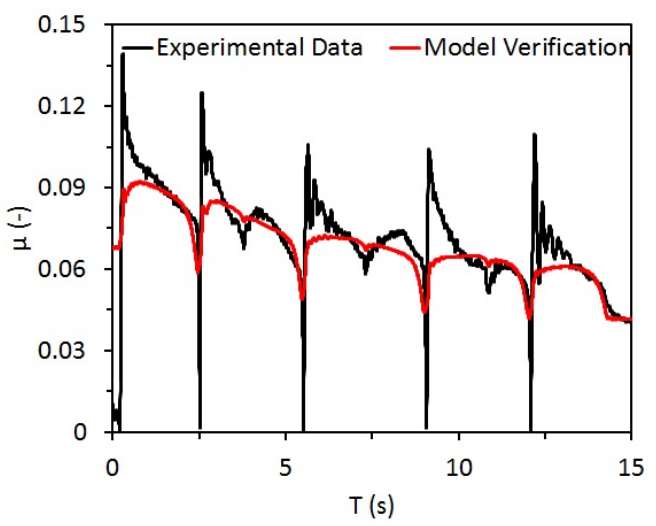

(C) brg17t1run10

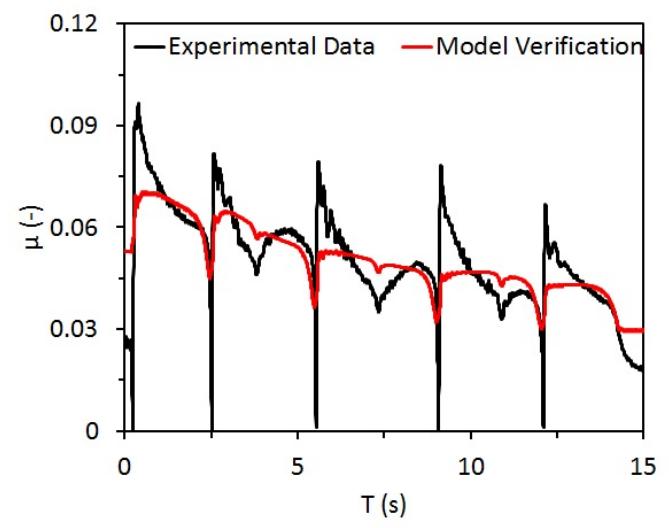

(E) brg17t1run12

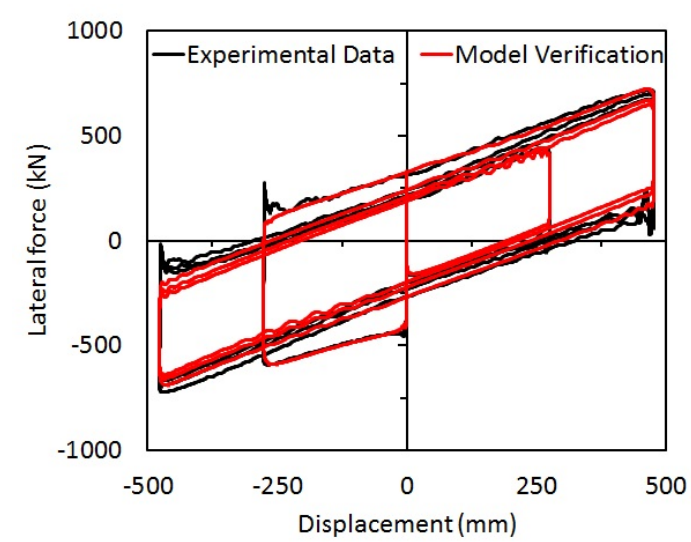

(B) brg17t1run9

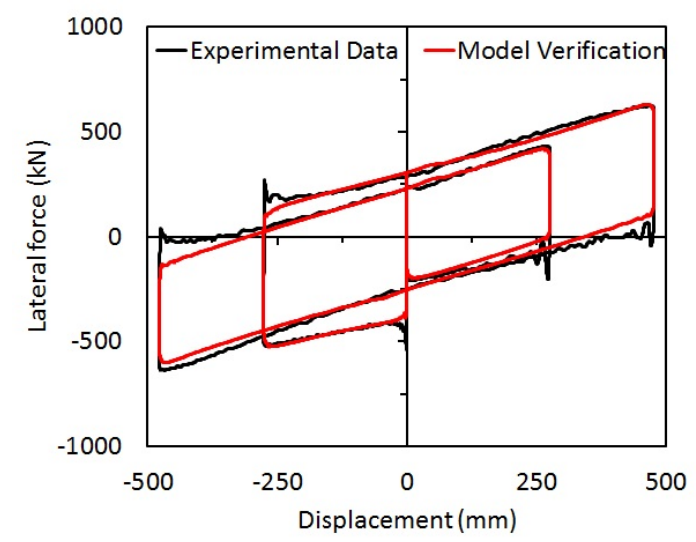

(D) brg17t1run10

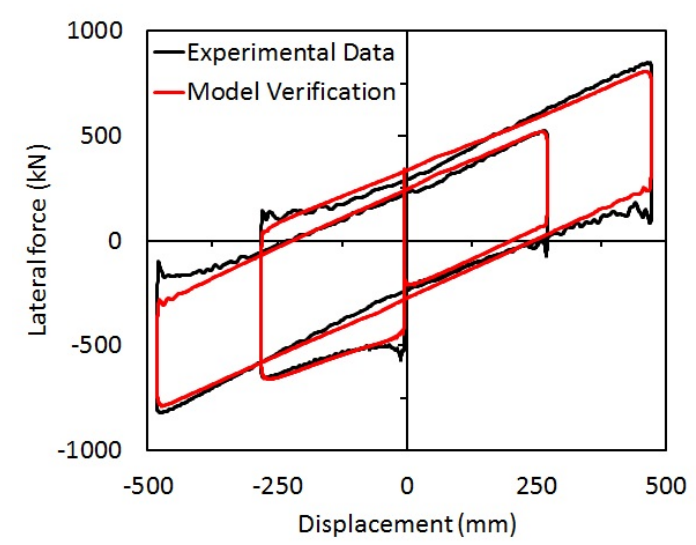

(F) brg17t1run12

FIGURE A.2: Bearing 17 


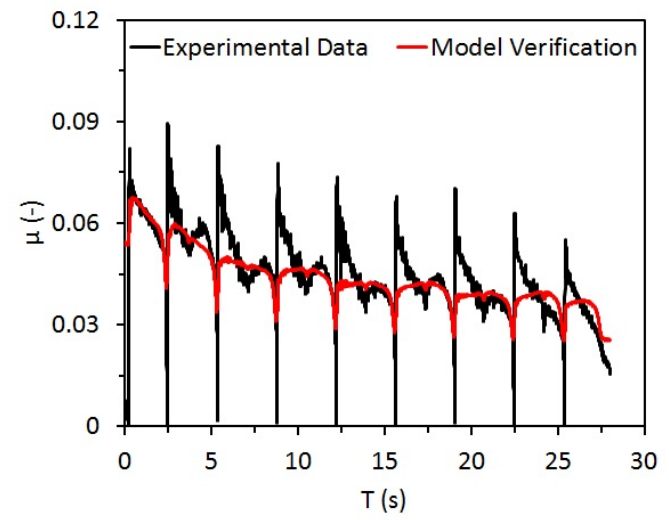

(A) brg18t1run5b

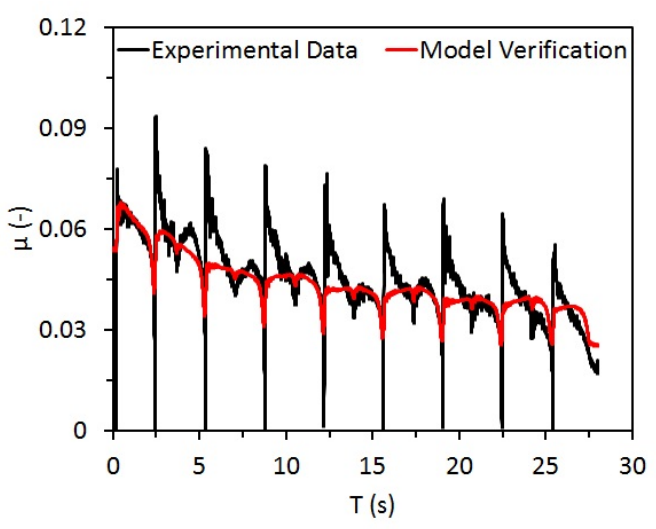

(C) brg18t1run9b

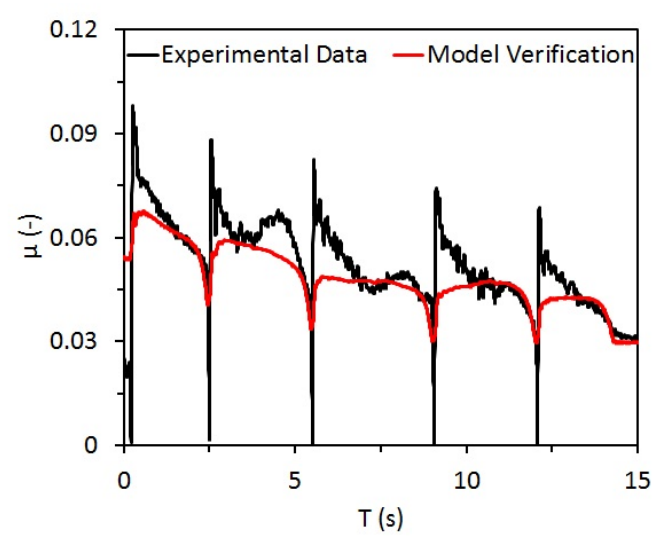

(E) brg18t1run10

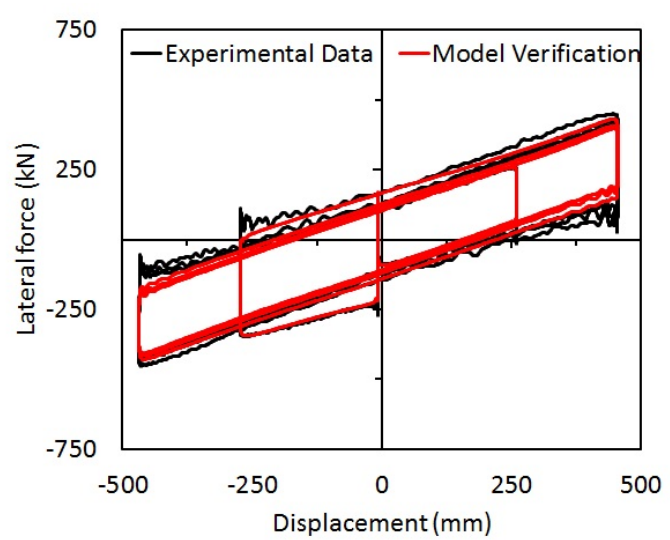

(B) brg18t1run5b

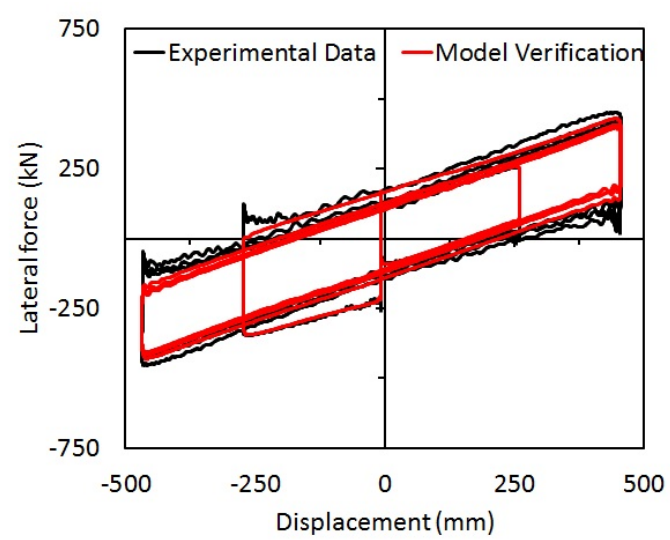

(D) brg18t1run9b

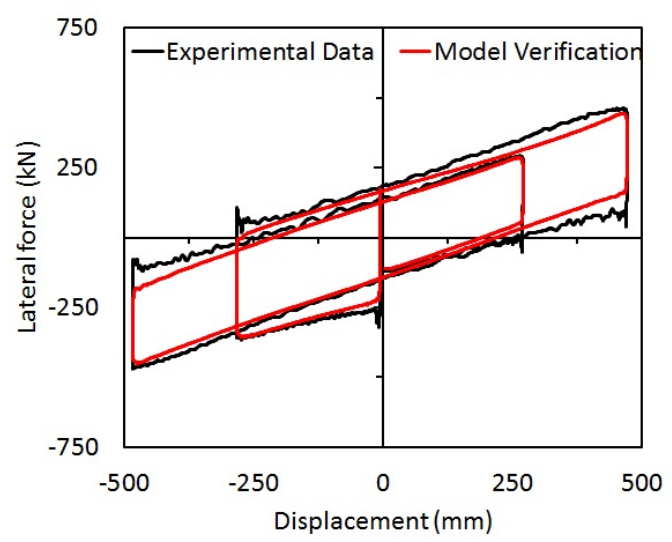

(F) brg18t1run10

FiguRE A.3: Bearing 18 


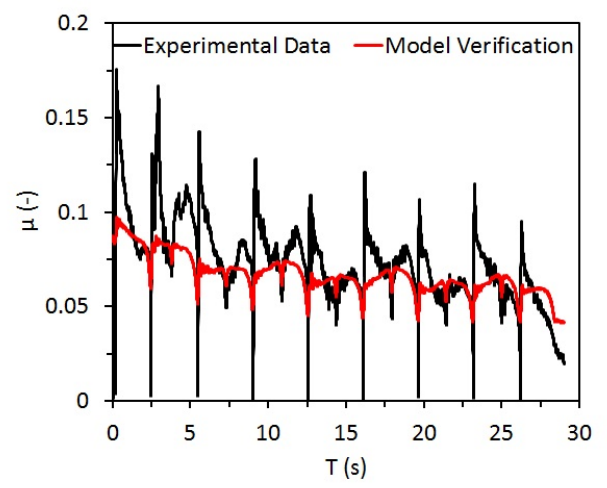

(A) brg19t1run5

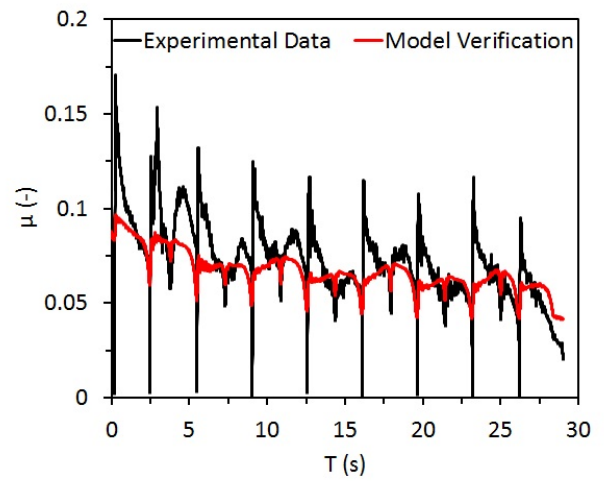

(C) brg19t1run9

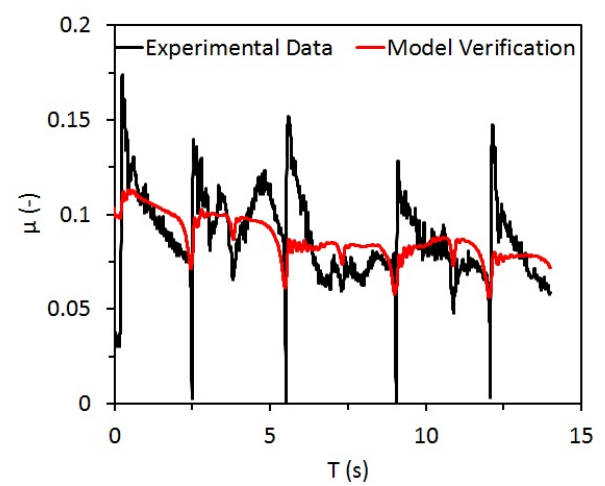

(E) brg19t1run10

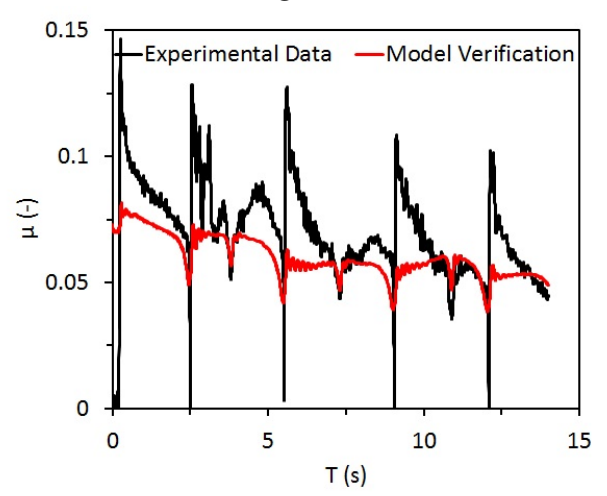

(G) brg19t1run11

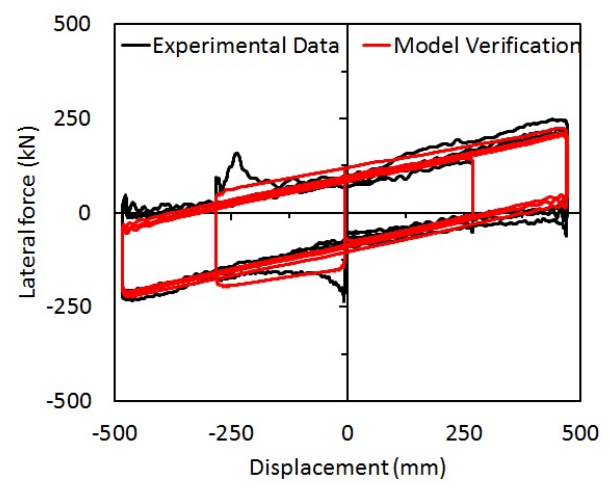

(в) brg19t1run5

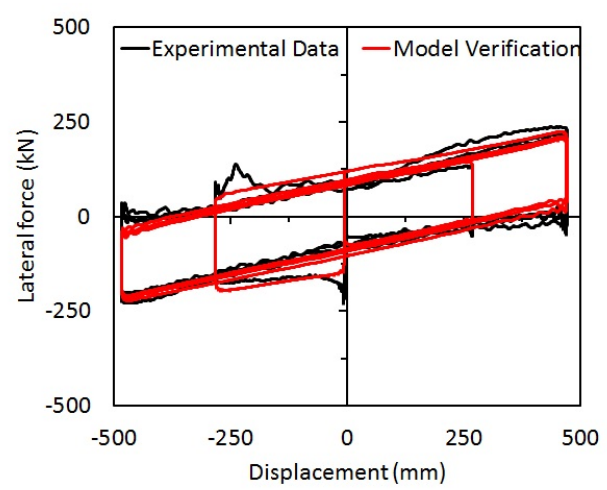

(D) brg19t1run9

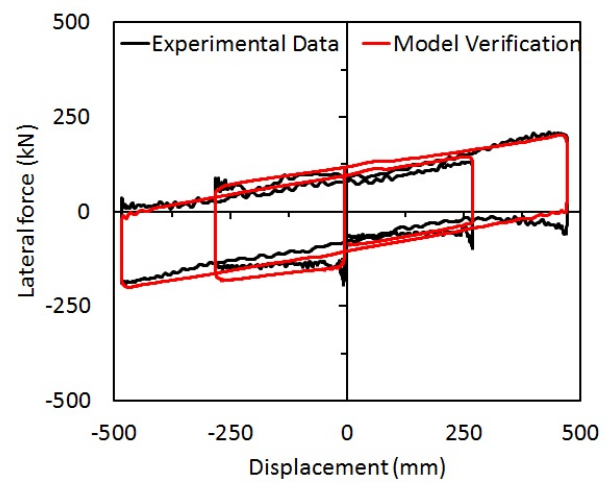

(F) brg19t1run10

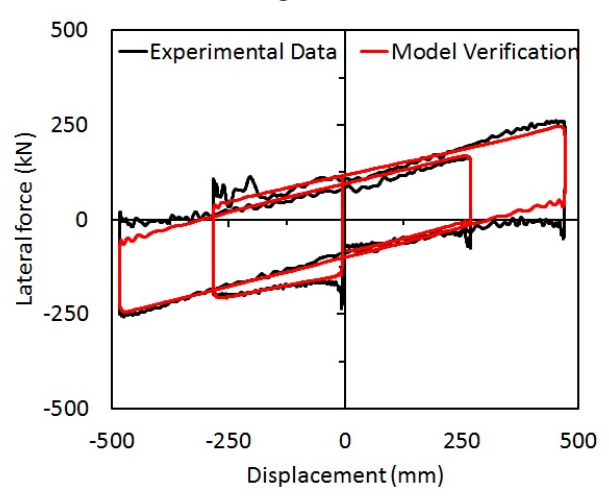

(H) brg19t1run11

FIGURE A.4: Bearing 19 


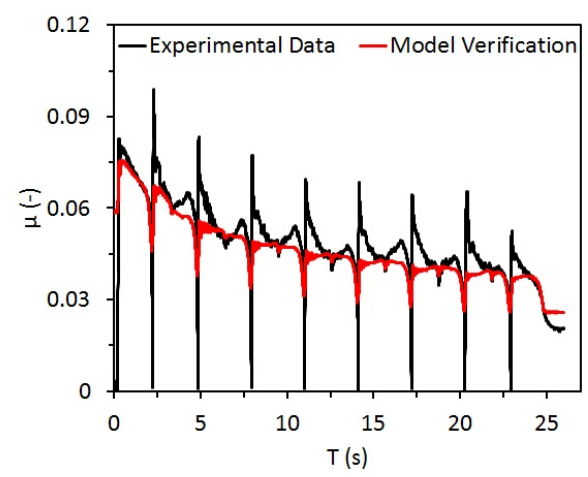

(A) brg20t1run5

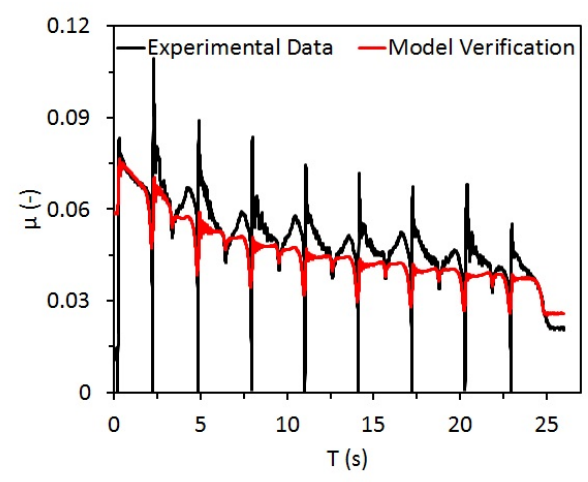

(C) brg20t1run9

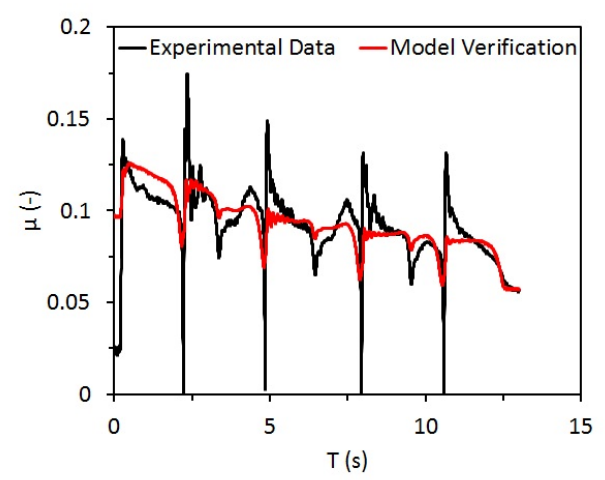

(E) brg20t1run10

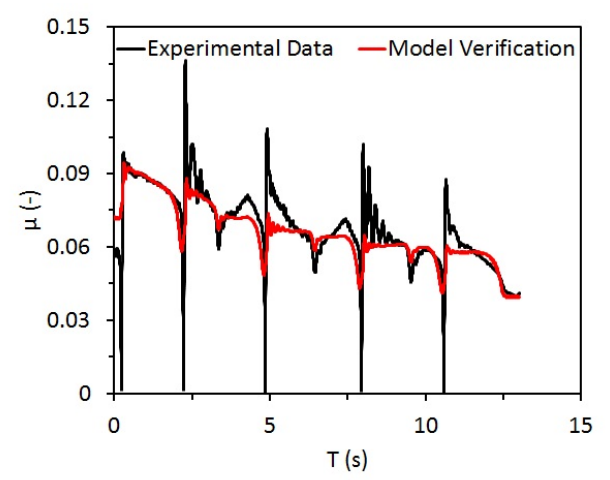

(G) brg20t1run11

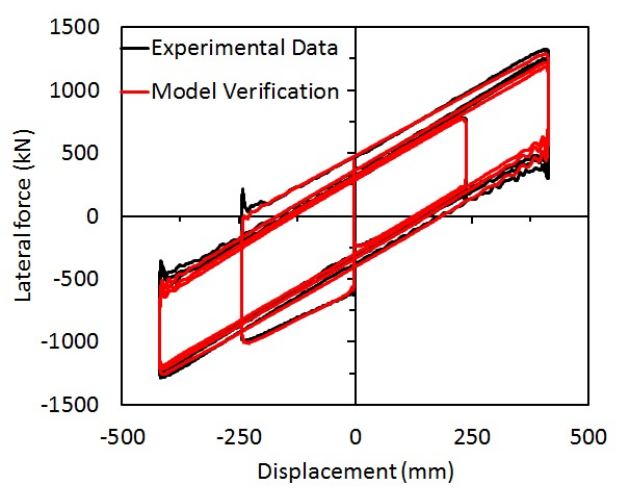

(в) brg20t1run5

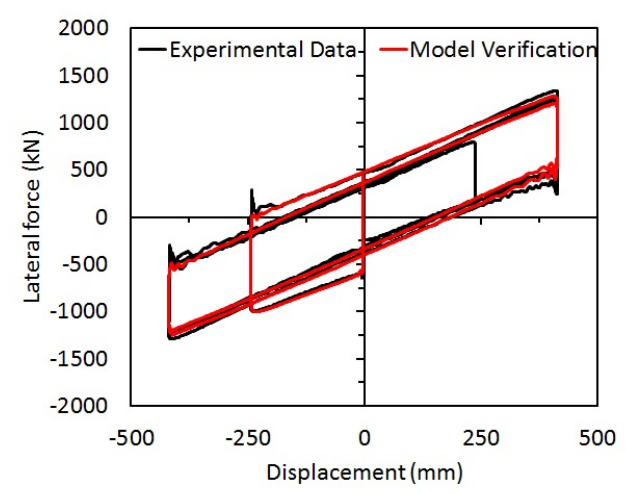

(D) brg20t1run9

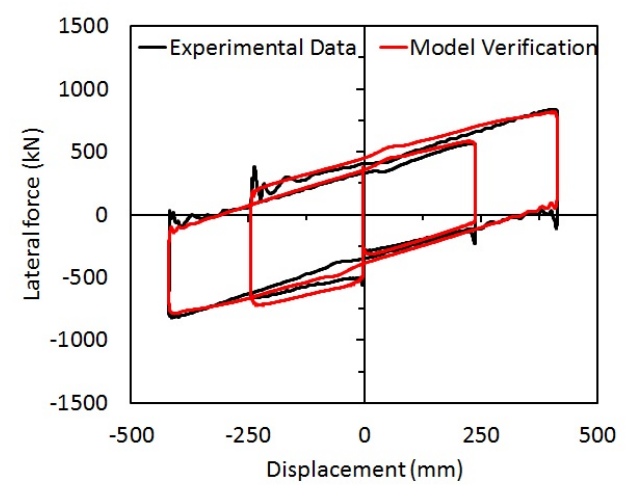

(F) brg20t1run10

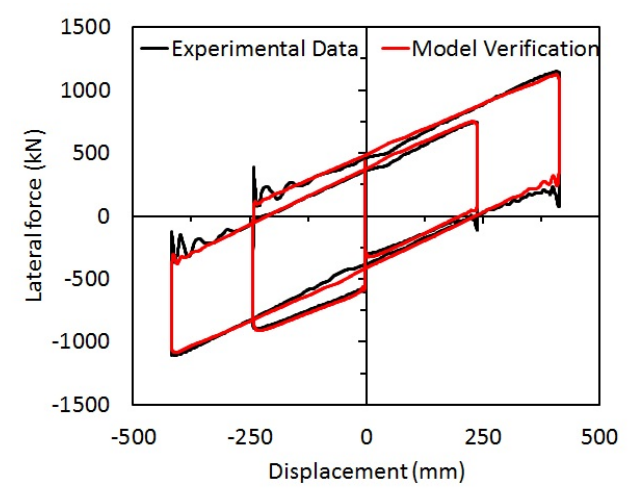

(H) brg20t1run11

FIGURE A.5: Bearing 20 


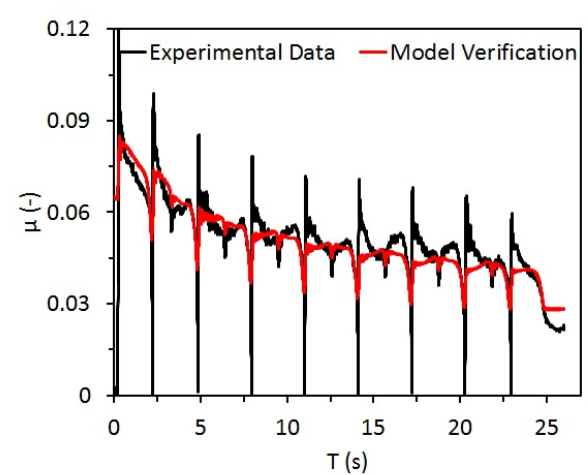

(A) brg21t1run5

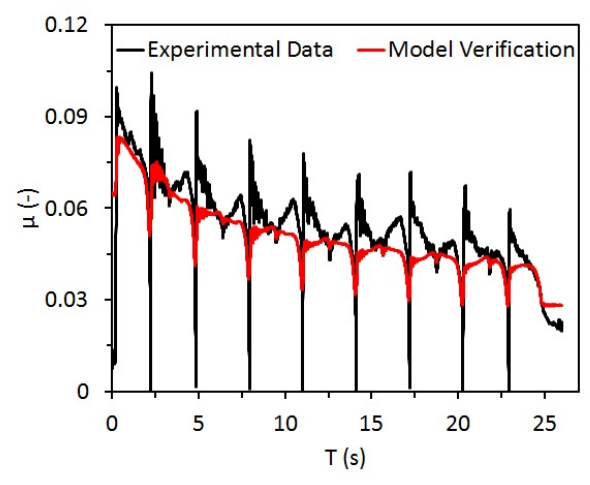

(C) brg21t1run9

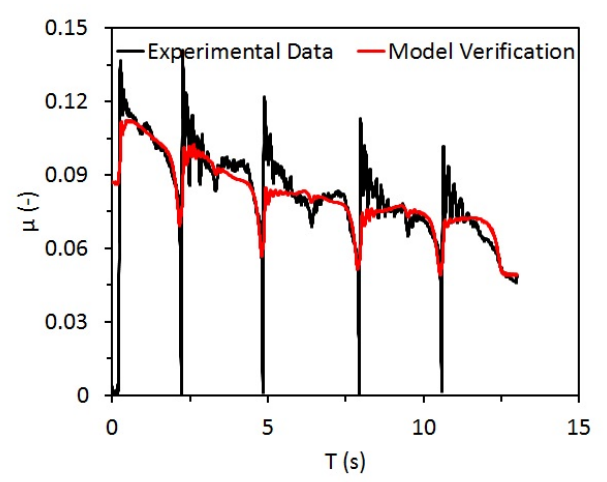

(E) brg21t1run10

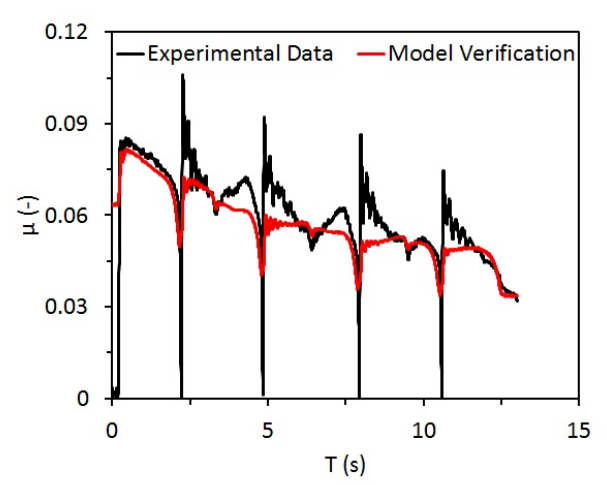

(G) brg21t1run11

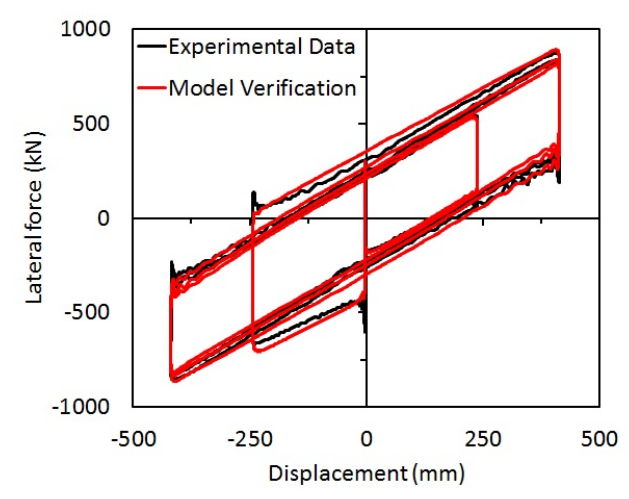

(в) brg21t1run5

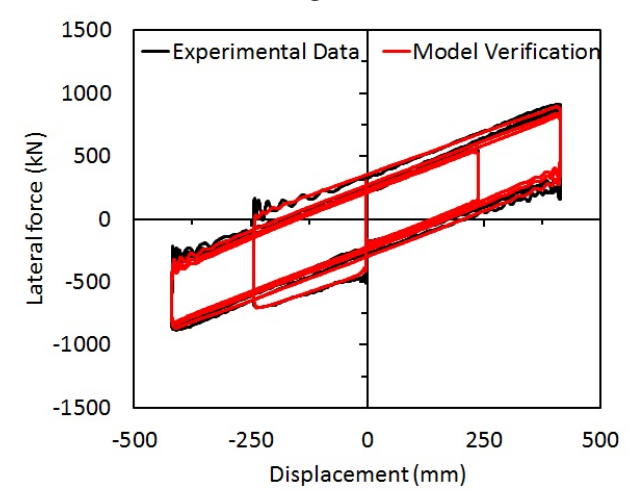

(D) brg21t1run9

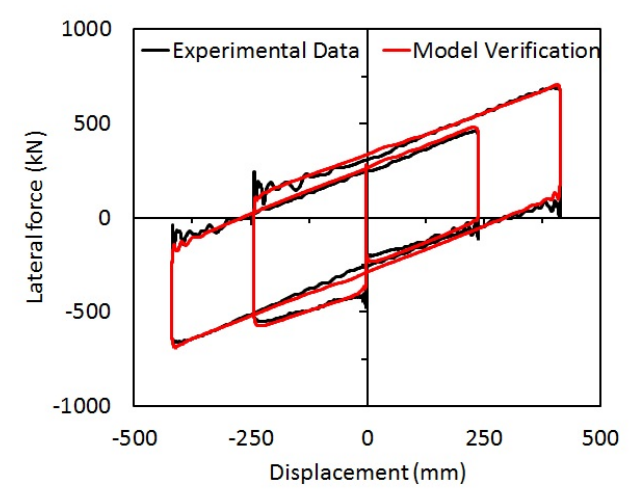

(F) brg21t1run10

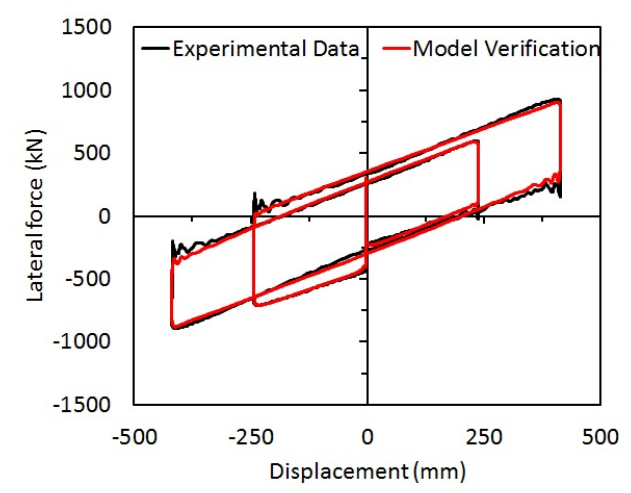

(H) brg21t1run11

FIGURE A.6: Bearing 21 


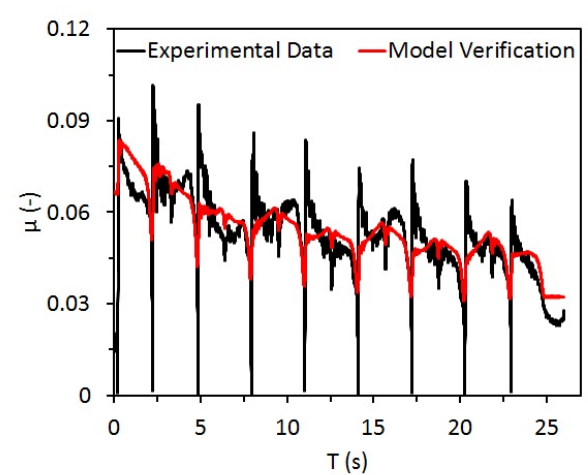

(A) brg22t1run5

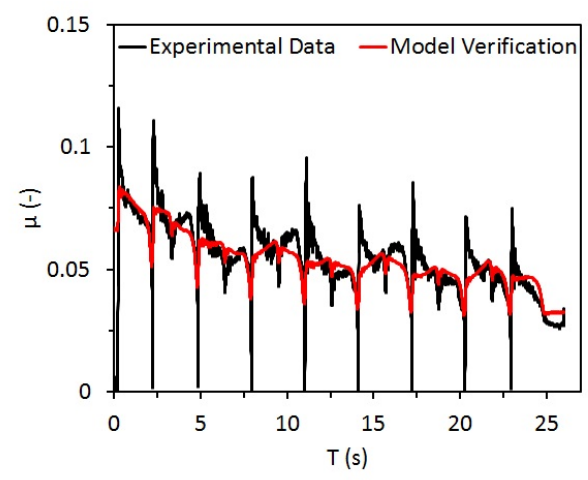

(C) brg22t1run9

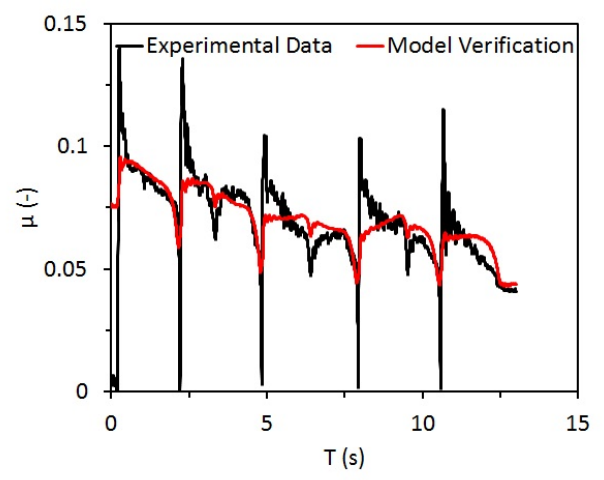

(E) brg22t1run10

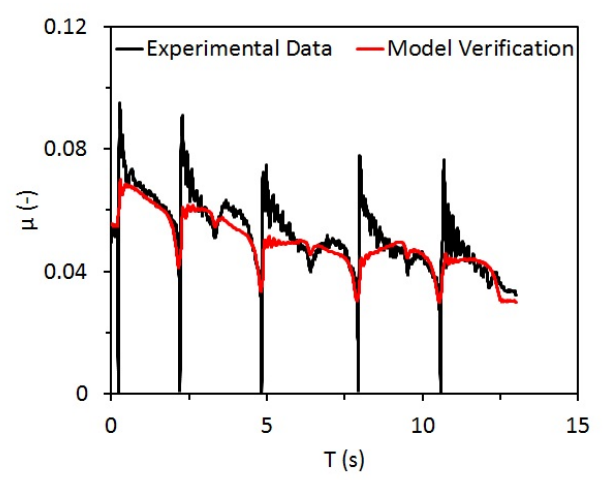

(G) brg22t1run11

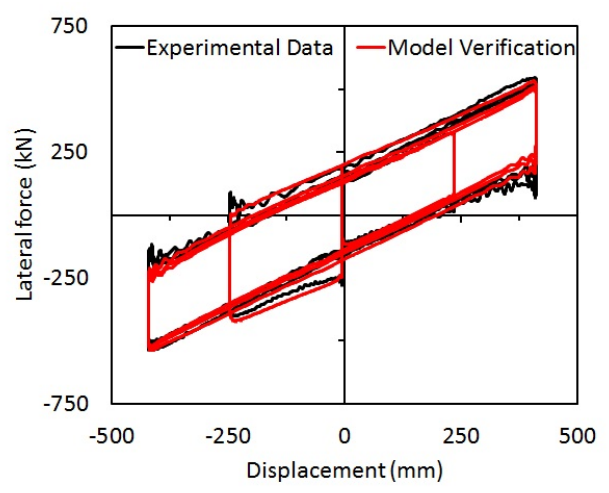

(в) brg22t1run5

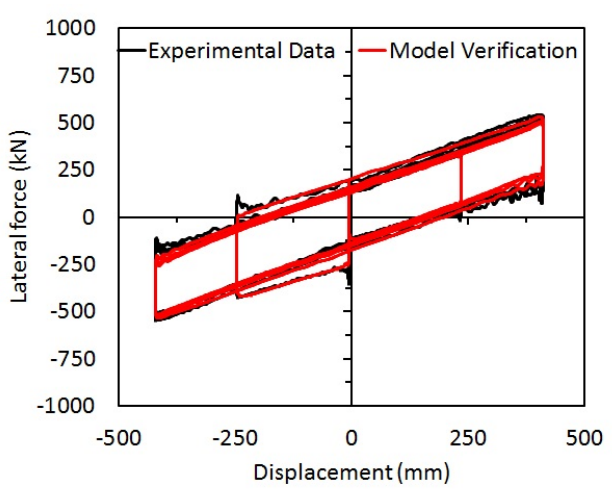

(D) brg22t1run9

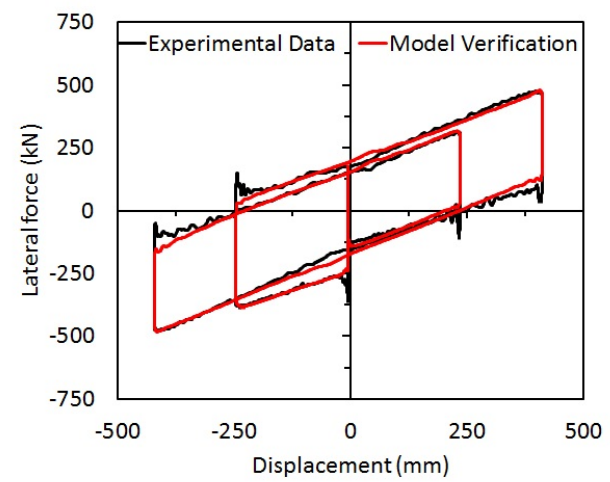

(F) brg22t1run10

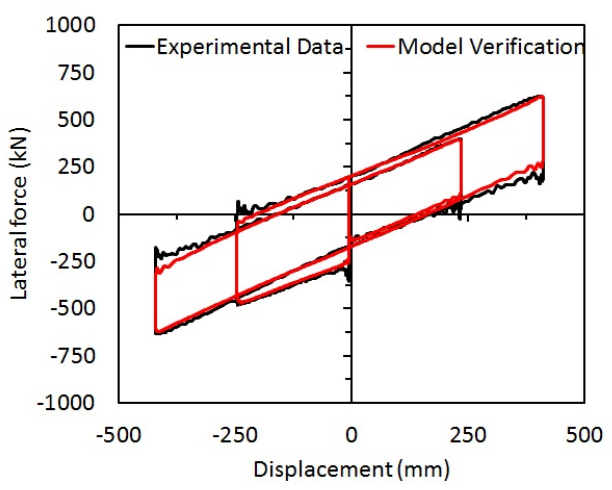

(H) brg22t1run11

FIGURE A.7: Bearing 22 


\section{Bibliography}

AASHTO (2012). AASHTO LRFD Bridge Design Specifications. ISBN: 9781560515234.

Almazán, José L. and Juan C. De la Llera (2002). "Analytical Model of Structures with Frictional Pendulum Isolators". In: Earthquake Engineering \& Structural Dynamics 31.2, pp. 305-332. ISSN: 00988847.

Almazán, José L., Juan C. De La Llera, and José A. Inaudi (1998). “Modelling Aspects of Structures Isolated with the Frictional Pendulum System". In: Earthquake Engineering E Structural Dynamics 27.8, pp. 845-867.

ASCE 7 (2010). Minimum Design Loads for Buildings and Other Structures. ISBN: 9780784410851.

Becker, Tracy C. and Stephen A. Mahin (2012). "Experimental and Analytical Study of the Bi-directional Behavior of the Triple Friction Pendulum Isolator". In: Earthquake Engineering \& Structural Dynamics 41.3, pp. 355-373. ISSN: 00988847.

Benzoni, Gianmario and Frieder Seible (1998). "Design of the Caltrans Seismic Response Modification Devicev (SRMD) Test Facility". In:

Benzoni, Gianmario, Giuseppe Lomiento, and Noemi Bonessio (2011). "Testing Protocols for Seismic Isolation Systems". In: Italian National Association of Earthquake Engineering.

Bondonet, Gaël and André Filiatrault (1997). "Frictional Response of PTFE Sliding Bearings at High Frequencies". In: Journal of Bridge Engineering 2.4, pp. 139-148. ISSN: 1084-0702.

Buckle, Ian G (2000). "Passive Control of Structures for Seismic Loads". In: 12th World Conference on Earthquake Engineering.

CEN/TC 340 (2006). EN 15129 Anti-Seismic Devices. Tech. rep.

Chang, By K C, Associate Members, and Asce Asce (1990). "Analytical model for sliding behavior of teflon-stainless steel interfaces". In: Journal of Engineering Mechanics 116.12, pp. 2749-2763.

Chang, Chia-Ming and Billie F. Spencer (2010). "Active base isolation of buildings subjected to seismic excitations". In: Earthquake Engineering \& Structural Dynamics 39.13, pp. 1493-1512. ISSN: 00988847.

Constantinou, Michalakis, Anoop Mokha, and Andrei Reinhorn (1990). "Teflon Bearings in Base Isolation II: Modeling". In: Journal of Structural Engineering 116.2, pp. 455-474. ISSN: 0733-9445.

Deb, Sajal K and Dilip K Paul (2000). "Seismic Response of Buildings Isolated by SlidingElastomer Bearing Subjected to Bi-directional Motion". In: 12th World Conference on Earthquake Engineering.

Dolce, M., D. Cardone, and F. Croatto (2005). "Analytical Model for Sliding Behavior of Teflon-Stainless Steel Interfaces". In: Bulletin of Earthquake Engineering.

Fenz, Daniel M. and Michael C. Constantinou (2006). "Behaviour of the Double Concave Friction Pendulum Bearing". In: Earthquake Engineering and Structural Dynamics. ISSN: 00988847. 
Kelly, James (1994). "The Implementation of Base Isolation in USA". In: Earthquake Engineering, Tenth World Conference.

Kumar, Manish, Andrew S. Whittaker, and Michael C. Constantinou (2015). "Measuring bias in structural response caused by ground motion scaling". In: Earthquake Engineering \& Structural Dynamics 44.November 2014, pp. 1409-1425. ISSN: 00988847.

Lomiento, G and G Benzoni (2017). "Testing Protocolsand Acceptance Criteria for Performance Characterization of Pendulum Isolators". In:

Lomiento, G, N Bonessio, and G Benzoni (2012). "Effects of Loading Characteristics on the Performance of Sliding Isolation Devices". In:

Lomiento, Giuseppe, Noemi Bonessio, and Gianmario Benzoni (2013). "Friction Model for Sliding Bearings under Seismic Excitation". In: Journal of Earthquake Engineering 17.8, pp. 1162-1191. ISSN: 1363-2469.

Lu, Lyan-ywan, Jain Wang, and Chao-chun Hsu (2006). "Sliding Isolation Using Variable Frequency Bearings for Near - Fault Ground Motions". In: 164, pp. 1-10.

Matsagar, Vasant A and R S Jangid (2008). "Base Isolation for Seismic Retrofitting of Structures". In: Practice Periodical on Structural Design and Construction 13.4, pp. 175185.

Mokha, A. S., M. C. Constantinou, and A. M. Reinhorn (1993). “Verification of Friction Model of Teflon Bearings under Triaxial Load". In: Journal of Structural Engineering 119.1, pp. 240-261. ISSN: 0733-9445.

Mokha, Anoop et al. (1991). "Experimental Study of Friction-Pendulum Isolation System". In: Journal of Structural Engineering 117.4, pp. 1201-1217. ISSN: 0733-9445.

Morgan, Troy A. and Stephen A. Mahin (2010). "Achieving Reliable Seismic Performance Enhancement Using Multi-Stage Friction Pendulum Isolators". In: Earthquake Engineering \& Structural Dynamics 39.13, pp. 1443-1461. ISSN: 00988847.

Mosqueda, Gilberto, Andrew S. Whittaker, and Gregory L. Fenves (2004). “Characterization and Modeling of Friction Pendulum Bearings Subjected to Multiple Components of Excitation". In: Journal of Structural Engineering 130.3, pp. 433-442. ISSN: 0733-9445.

Tsai, C. S. (1997). "Finite Element Formulations for Friction Pendulum Seismic Isolation Bearings". In: International Journal for Numerical Methods in Engineering 40.1, pp. 2949. ISSN: 0029-5981.

Tsai, C.S. et al. (2008). "Finite element formulation and shaking table tests of directionoptimized-friction-pendulum system". In: Engineering Structures 30.9, pp. 2321-2329. ISSN: 01410296.

Tsopelas, Panos et al. (1996). "Experimental study of bridge seismic sliding isolation systems". In: EarthquakeEngineering \& Structural Dynamics 25.1, pp. 65-78.

Warn, Gordon P and Keri L Ryan (2012). "A Review of Seismic Isolation for Buildings: Historical Development and Research Needs". In: Buildings 2, pp. 300-325. ISSN: 2075-5309.

Zayas, Victor A. (1987). The FPS Earthquake Resisting System Experimental Report. Tech. rep. Berkeley, Calif. :

Zayas, Victor A., Stanley S. Low, and Stephen A. Mahin (1990). "A Simple Pendulum Technique for Achieving Seismic Isolation”. In: Earthquake Spectra 6.2, pp. 317-333. ISSN: 8755-2930. 